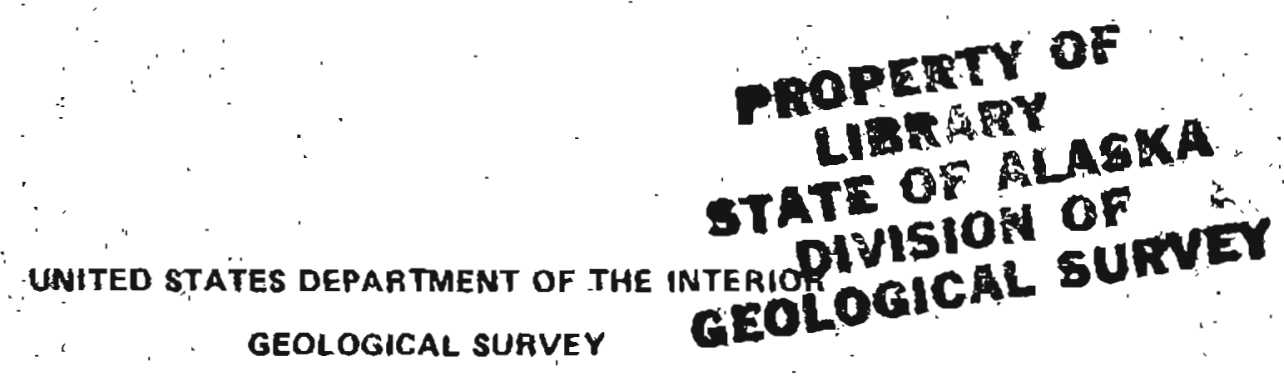

CATALOG OF EARTHQUAKES IN SOUTHERN ALASKA

JANUARY-MARCH 1980

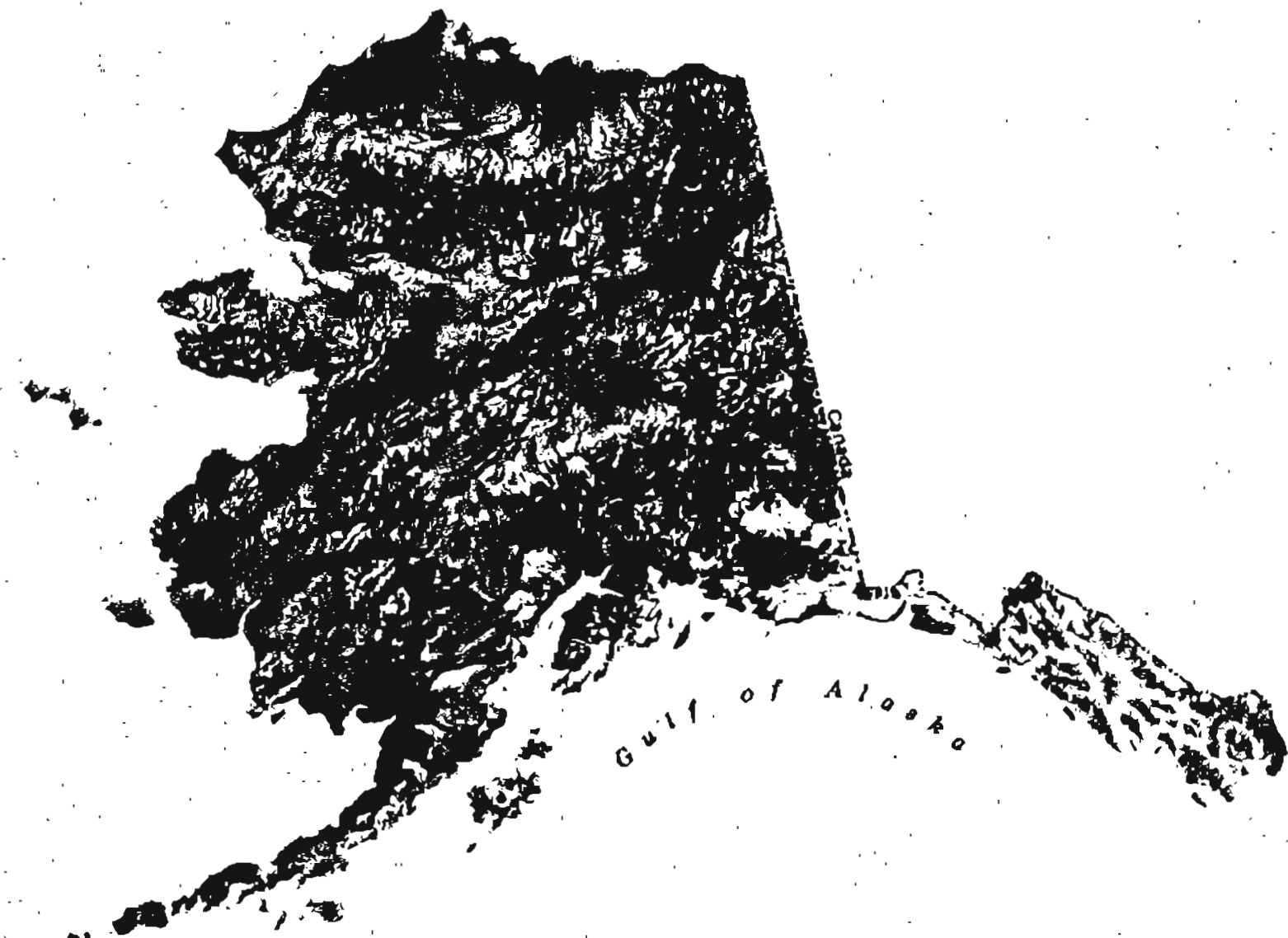

OFEAFIE PEPCRT 80.1253

This raport is proliminary and has not been adled or reviewed for conformity with Geological Survey standards and nomendature

Menlo Park, California

1980 


\section{CATALOG OF EARTHQUAKES IN SOUTHERN ALASKA JANUARY-MARCH 1980}

C. D. Stephens, K. A. Fogleman, J. C. Lahr, S. M. Helton, R. S. Cancilla, Roy Tam, J. A. Freiberg

\section{CONTENTS}

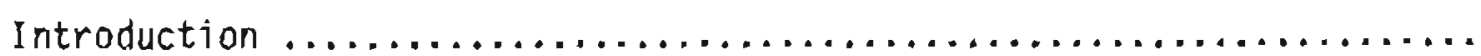

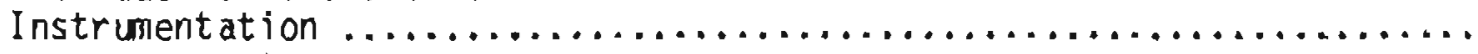

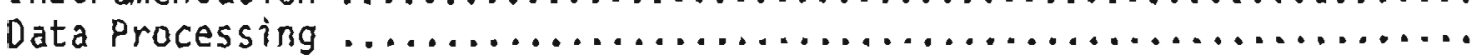

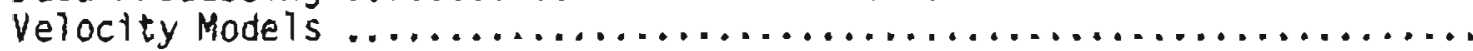

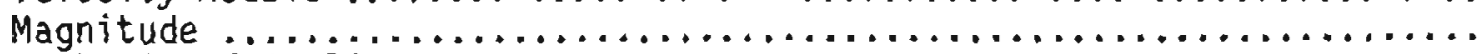

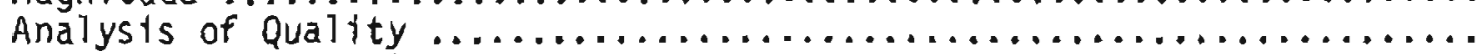

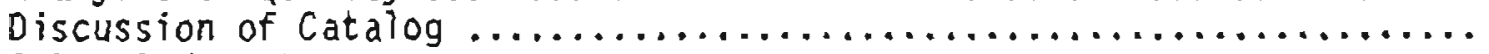

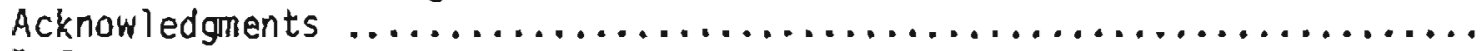

\section{ILLUSTRATIONS}

Figure I Map showing principal seismograph

stations used in locating earthquakes ............. 3

2 B lock diagram of the USGS

telemetered seismograph system

3 System response curves of typical

USGS telemetered seismograph stations ............. 7

4 Picture of a typical

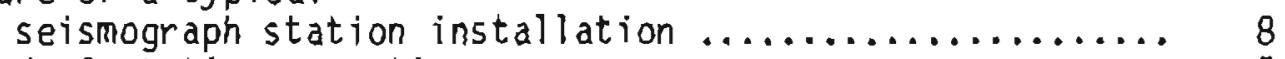

5 Record of station operation

6 Map showing earthquake

epicenters reported in the appendix

7 Map of epicenters for earthquakes with

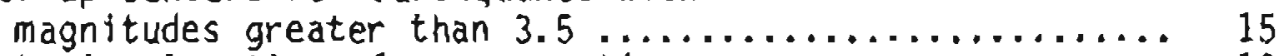

8 Map showing location of cross sections ................... 19

9 Cross sections showing distribution of earthquake hypocenters listed in the appendix ..... 20

\section{TABLES}

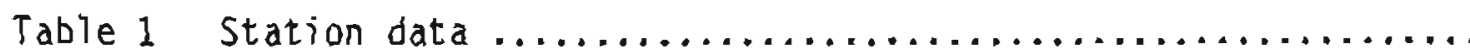

\section{APPENDICES}

Appendix Southern Alaska earthquakes, first quarter $1980 \ldots . . . \ldots .$. 
The National Center for Earthquake Research of the U.S. Geological Survey (USGS) began a program of telemetered seismic recording in south-central Alaska in 1971. The principal objectives of this program have been to use data recorded by this network to precisely locate earthquakes in the active seismic zones of southern Alaska, delineate selsmically active faults, assess seismic risk, document potential premonitory earthquake phenomena, investigate current tectonic deformation, and study the structure and physical properties of the crust and upper mantle. A task fundamental to all of these goals is the routine cataloging of earthquake parameters for earthquakes located within and adjacent to the seismograph network.

The initial network of 10 stations, 7 around Cook Inlet and 3 near Valdez, was installed in 1971. Each surmer since then additions or modlfications to the network have been made. By the Fall of 1973 , 26 stations extended from western Cook inlet to eastern Prince William Sound, and 4 stations were located between Cordova and Yakutat. A year later 20 additional stations were installed. Thirteen of these were placed along the eastern Gulf of Alaska with support from the National Oceanic and Atmospheric Administration (NOAA) under the Outer Continental Shelf Environmental Program to investigate the seismicity of the outer continental shelf, a region of interest for oil exploration. During the subsequent years the region covered by the network has remained relatively fixed while effort has been made to improve the instrumentation and installation of the stations in order to make them more reliable.

The locations of the stations of the USGS seismograph network are plotted in Figure 1 and listed in Table 1 along with the additional stations from which readings were obtained. Each USGS station has a single, verticalcomporent seismometer. The stations GLB, PNL, RDT, SKN, and VLZ also have north-south- and east-west-oriented horizontal seismometers.

This earthquake catalog presents origin times, focal coordinates and magnitudes for 1376 shocks occurring in the first quarter of 1980 . Readings from a total of 66 stations were used to locate the shocks, including 11 stations operated by the NOAA Alaska Tsunami Warning Center (formeriy Palmer Observatory), 5 stations operated by the Geophysical Institute of the Universtty of Alaska (U. of $A_{\text {. }}$ ), and 5 stations operated in southwest Yukon Territory by the Department of Energy, Mines and Resources, Canada.

Earthquakes in south-central Alaska as small as magnitude 3.0 have been routinely located by the National Earthquake Information Service of the USGS and its predecessor since the great Alaska earthquake of 1964 and are published in the reports "Preliminary Determination of Epicenters" (PDE). In contrast, the shocks included in this catalog are as small as magnitude 1.0 and most are smaller than magnitude 3.0. Data for the larger historic earthquakes that occurred in south-central Alaska through 1975 have been tabulated by Meyers (1976).

\section{INSTRUMENTATION}

The instrumentation in the USGS seismograph network is illustrated in the block diagram in Figure 2. Data from each seismometer are telemetered to the NOAA Alaska Tsunami Warning Center in Palmer. The standard equipment at each field station includes a vertical seismometer with a natural frequency of 


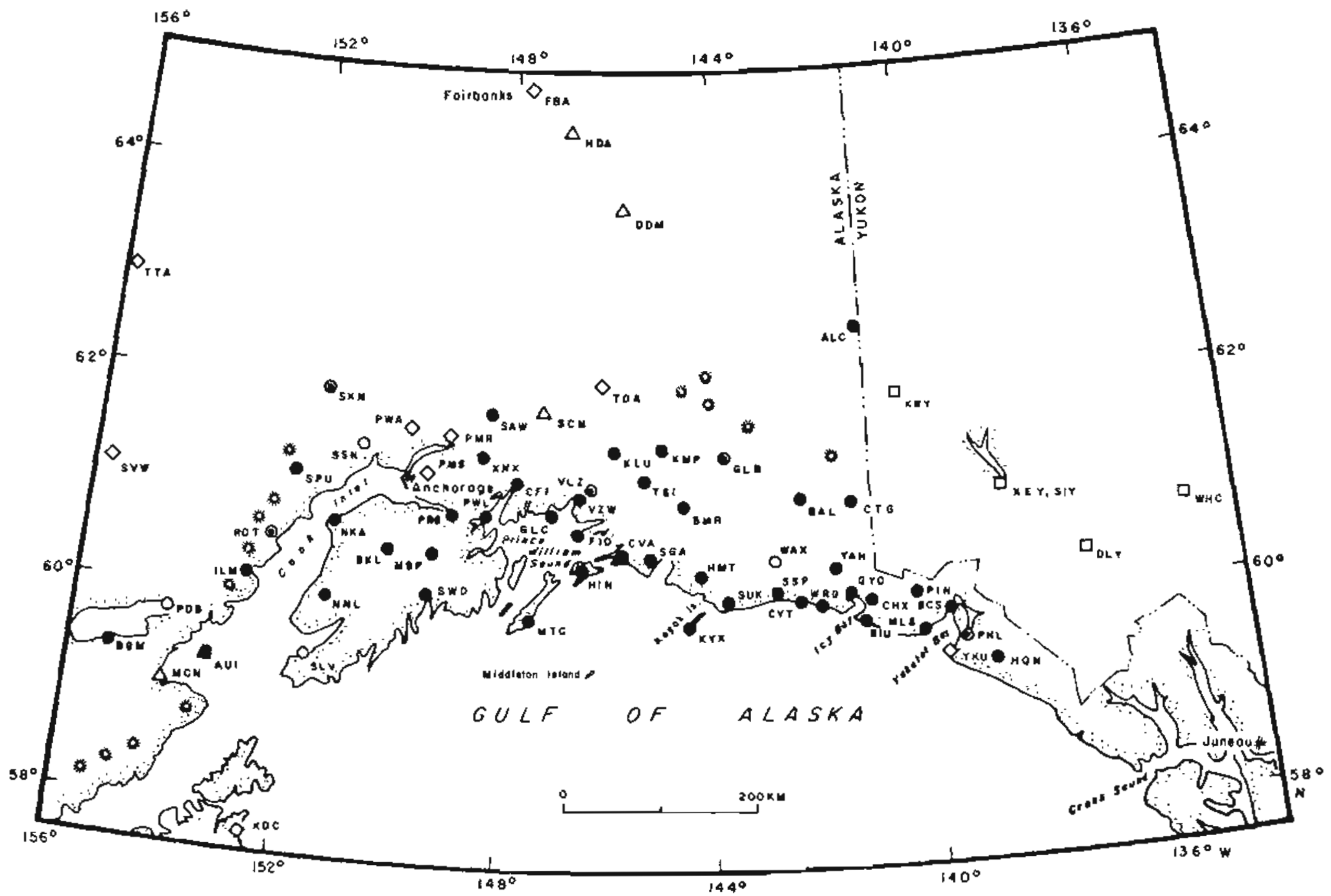

Figure 1. Map showing the locations of all USGS seismograph stations in southern Alaska and other stations used in the preparation of this catalog. The symbois are as follows: solid circles, vertical component USGS seismograph; circles with dots, three-component USGS seismographs; open circles, USGS stations not reporting during this quarter; diamonds, NOAA stations; triangles, Univ. of Alaska stations; squares, Dept. Energy, Mines and Resources, Canada. Quaternary volcanoes (after King, 1969) are indicated by stars. 
Table 1. Station Data

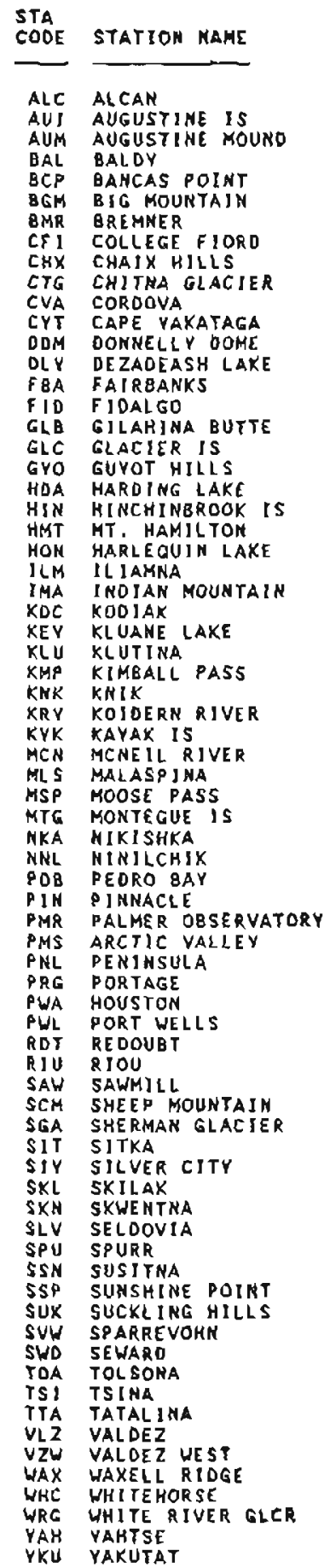

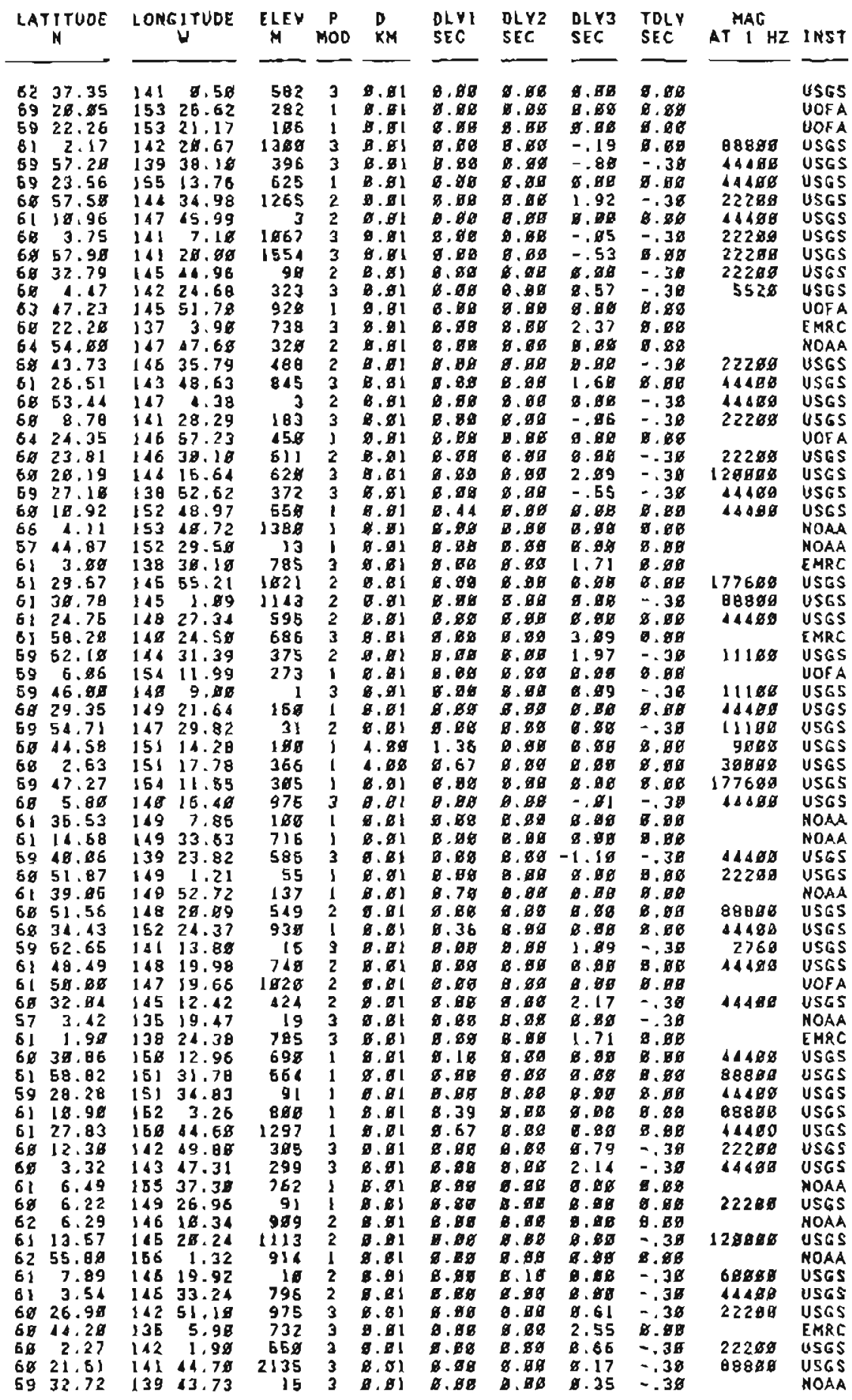

This table lists geographic coordinates and other pertinent information for stations used in the preparation of this catalog. P-MOD is the number of the $P$-wave velocity model assigned to the station (see text), where the numbers 1,2 , and 3 correspond to the western, central, and eastern models. $D$ is the thickness of the low-velocity surficial sedimentary layer in kilometers assigned in the calculation of travel-times to a given station. DLY1-3 are the station P-phase travel-time delays in seconds. TDLY is the telephone line delay in seconds. The magnification (MAG) of the vertical seismograph component is given at $1 \mathrm{~Hz}$. The institutions (INST) operating the stations other than the USGS are the NOAA Alaska Tsunami Warning Center, the Geophysical Institute of the University of Alaska (UOFA) and the Department of Energy, Mines and Resources, Canada (EMRC). 


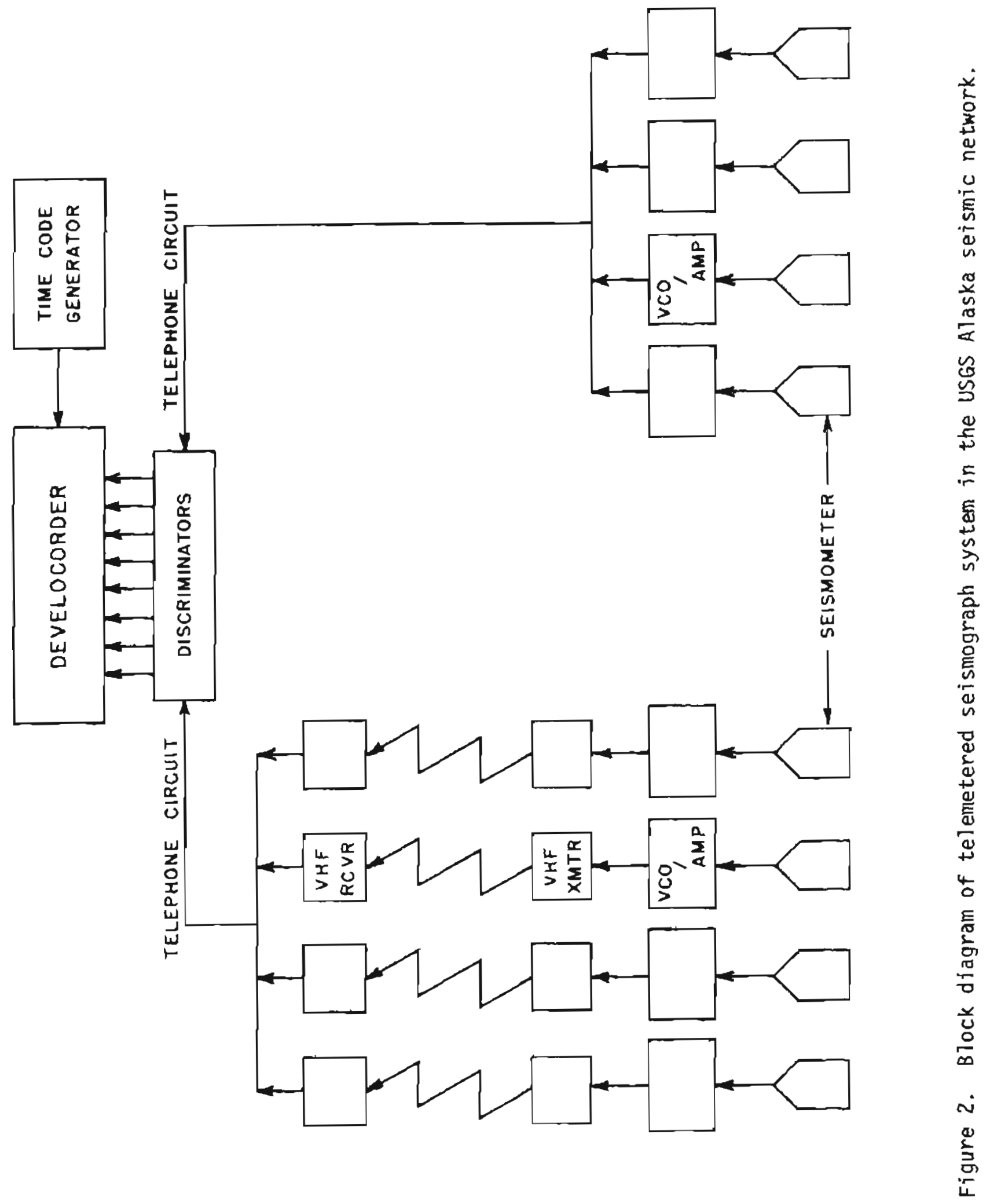


$1.0 \mathrm{~Hz}$ (Mark Products, Model $\mathrm{L}-4$ ), a package consisting of a pre-amplifier and a voltage-controlled oscillator (VCO model NCER 202, or AIVCO) and "air-cell" storage batteries (MCGraw-Edison, Model 5T-2-1000). The recentiy developed AlvCO units (Rogers and others, 1980) have been installed at nearly all of the USGS stations in southern Alaska. These crystal-referenced units have an automatic gain-ranging capability and provide daily information on the gain setting, geophone response, battery voltage, station identification and temperature. Data are telemetered via a combination of leased telephone circuits and VHF (162-174 MHz) radio links. The radio equipment consists of low-power transmitters $(100 \mathrm{~mW})$ and receivers adapted from $\mathrm{HT}-200$ Motorola handie-talkie transceivers. Yagi antennae with 9 db directional gain (Scala, Model CAS-150) are used. At some sites where AC power is available, basestation radio receivers (G.E. Model R46AP66B) with greater sensitivity and reliability are used. The central recording facility incorporates a bank of discriminators (NCER 3101 or Develco Model 6203), four $16 \mathrm{~mm}-\mathrm{fi} 3 \mathrm{~m}$ multichannel oscillographs (Teledyne Geotech Develocorder, Model 40000), a 14-channel analog tape recorder (Bell and Howel? Model VR3700B), and a time-code generator (Datum, Model 9100).

The principle of operation is as follows: The seismometer translates movement of the ground into an electrical voltage that is fed into the amplifier/vCO unit where the amplified voltage causes the frequency of an audio-band oscillator to fluctuate about its center frequency. The frequency-modulated (FM) tone from the amplifier/VCO unit is carried directly by voice-grade teleohone circuit to the recording site or alternately is fed through a VHF radio link onto a telephone circuit. At the recording site the FM seismic signal is demodulated by a discriminator. The demodulated signal, which is simply an amplified form of the initial signal from the seismometer, is recorded photographically on a multichannel osclilograph, together with time marks from a crystal-controlled chronometer. Twenty-four hours of data for 18 stations can be recorded on a single $43 \mathrm{~m}$-long roll of $16 \mathrm{~mm}$ film.

Signals from more than one seismograph can be transmitted on a single telephone circuit by employing VCO units with different center frequencies. In the standard configuration there is a $340 \mathrm{~Hz}$ separation between center frequencies and a fixed bandwidth of $250 \mathrm{~Hz}$. Up to eight seismic channels with center frequencies ranging from 680 to $3060 \mathrm{~Hz}$ may be placed on a single voice-grade telephone circuit.

Figure 3 illustrates the response characteristics of the entire seismic system from seismometer to $f i l m$ viewer. The response level at each station is adjusted in steps of 6 decibels so that the ambient seismic noise produces a small deflection of the trace on the $f 11 \mathrm{~m}$. As a result, the actual response for an individual station may differ from that of the typical station by a factor of 2, 4, 8, etc. The magnification of the typical station is about $6 \times 10^{4}$ at $\mathrm{I} \mathrm{Hz}$ and 106 at $10 \mathrm{~Hz}$. The gain of a station that has an AlvCO unit is automatically reduced by a factor of 10 when the fluctuations of the FM signal exceed a preset threshold.

The installation of a typical radio-linked station is shown in Figure 4. Degradation or interruption of data transmission due to inclement weather conditions is a major problem during the winter months. A record of station operation is presented in Figure 5.

\section{DATA PROCESSING}

The $16 \mathrm{~mm}$ films (four per day) are mailed weekly to Menlo Park where the 


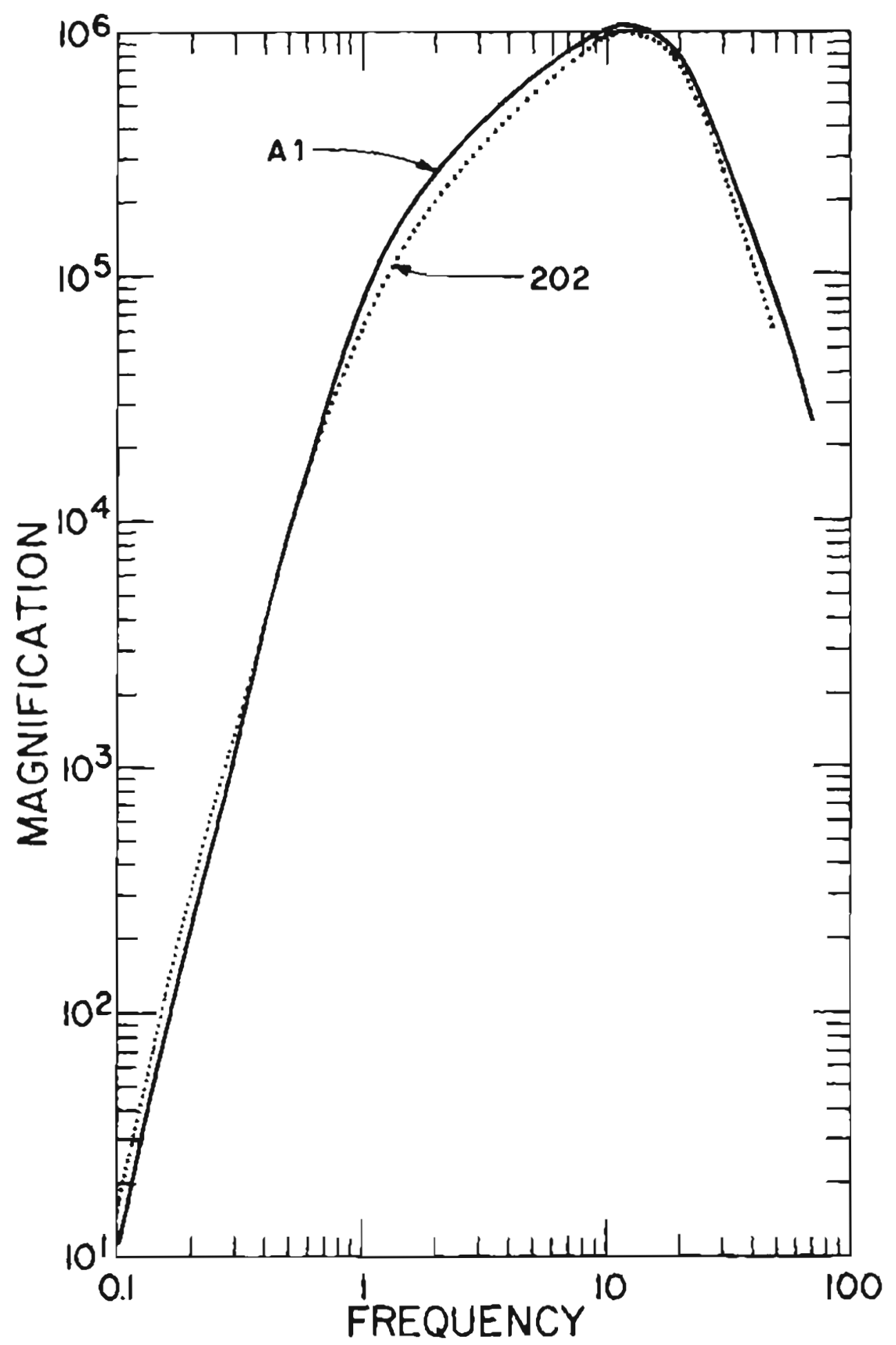

Figure 3. System response curves for typical USGS A7aska seismographs that incorporate the AlVCO unit (solid curve) and the older VCO model NCER 202 unit (dotted curve). 


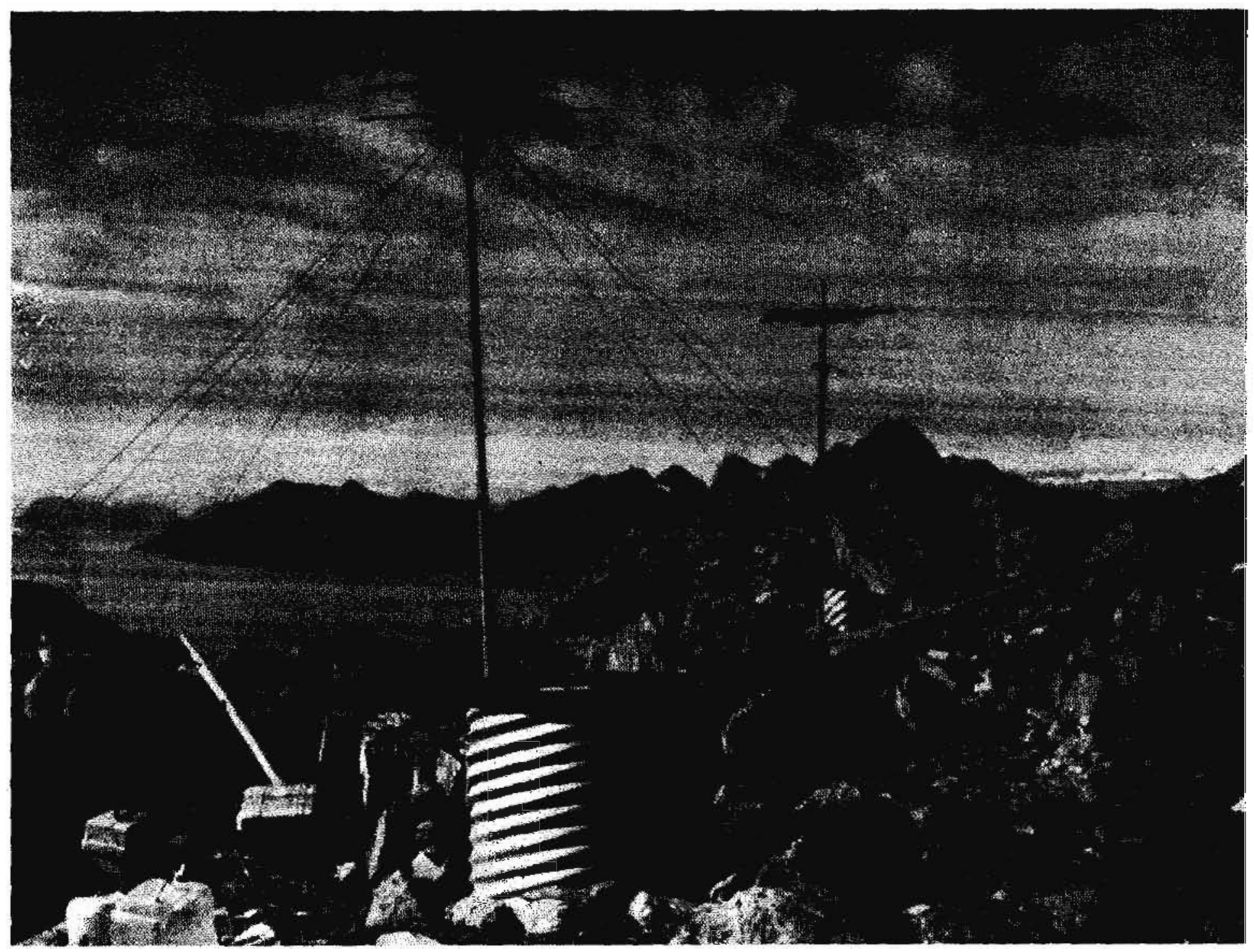

Figure 4. Installation of a typical seismograph station (VZW). VCo/amplifier unit, radio transmitter, and batteries are housed in a 30-inch diameter culvert partialiy set in the ground at the base of the antenna. Seismometer is buried in the ground about 30 meters from the culvert. Photograph was taken prior to installation of new antenna masts (seen lying on the ground). A repeater site is seen in the background. View is southwest toward Glacier Island (at left center of picture). 


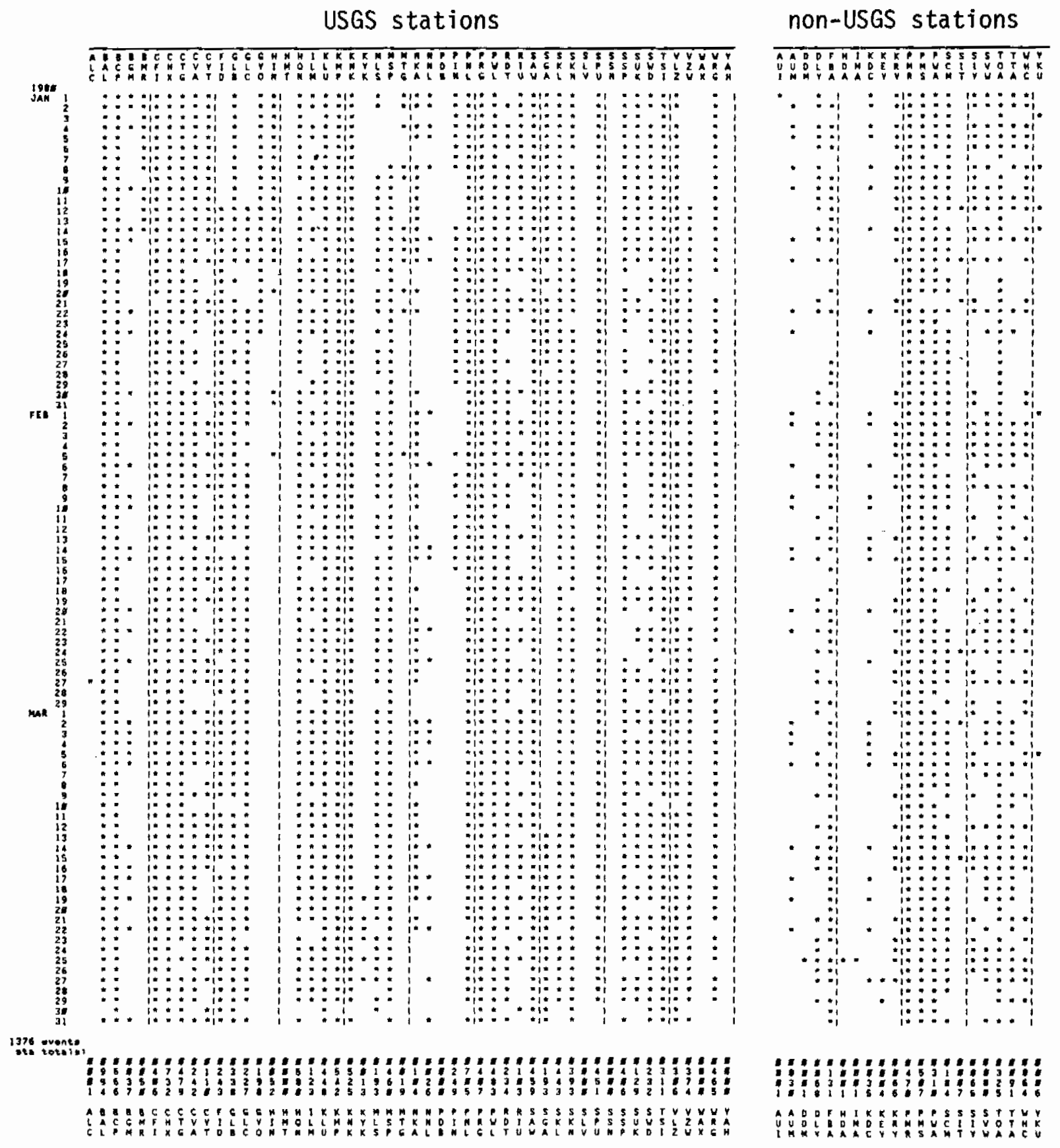

Figure 5. Record of station operation during the first quarter of 1980 . This figure indicates the days on which arrival times were obtained for locating earthquakes. The totals at the bottom indicate the number of events for which arrival times were obtained at each station. 
seismic data are processed by the following multi-step routine:

1. Scanning. The scan film, which has 18 stations distributed throughout the network is scanned to identify and note times of all seismic events whether of Tocal, regional, or teleseismic origin.

2. Timing. For the "well-recorded" local earthquakes identified in the scanning process, the following data are read from each station: $P$ - and Swave arrival times, direction of first motion, duration of signal in excess of a given threshold amplitude, and period and amplitude of maximum recorded signal. The criterion for choosing earthquakes to be timed is the duration of the signal, which is related to the magnitude. The network is divided into three regions--western, central and eastern--bounded approximately by 1560 to $1500 \mathrm{~W}, 1500$ to $1450 \mathrm{~W}$ and 1450 to $1380 \mathrm{~W}$, respectively. In the western and central regions, only events with signal durations longer than $80 \mathrm{~s}$ and $20 \mathrm{~s}$, respectively, are timed. In the eastern region, all earthquakes which are recorded by at least three stations and for which at least four clear arrivals can be read are timed. This criterion was established to facilitate processing the large number of earthquakes which are recorded by the network.

Timing is done by projecting the seismic traces onto a table and digitizing the onsets of the $P_{-}$and 5 -phases. The output from the digitizer, in the form of $x-y$ data pairs on punched computer cards, is converted into phase data by computer using the program DIGIT3 (written by P. Ward and W. Elisworth for use within the U.S. Geological Survey).

3. Initial computer processing. The phase data from the flims is batch processed by computer using the program HYPOELLIPSE (Lahr, 1980) to obtain origin times, hypocenters, magnitudes and, if desired, first-motion plots for fault-plane solutions.

4. Analysis of initial computer results. Each hypocentral solution is checked for large travel-time residuals and for a poor spatial distribution of stations. Arrival times that produce 7 arge residuais are re-read. For shocks with a poor distribution of stations, readings from additional stations outside the USGS network are sought.

5. Final computer processing. The poor hypocentral solutions are rerun with corrections and the new solutions are checked for large residuals that might be due to remaining errors. Corrections are made as required before the final computer run is made.

The earthquake locations are based on $P$ and $S$ arrivals. S arrivals are important for determining depths of events in the Benioff zone beneath cook Inlet. Unfortunately for some large events, $S$ cannot be read at any station because the traces on the film overlap each other or are too faint to follow.

The HYPOELLIPSE computer program determines hypocenters by minimizing differences between observed and computed travel-times through an iterative least-squares scheme. In many respects the program is similar to HYPO71 (Lee and Lahr, 1972), which has been used in the preparation of catalogs of central Ca]ifornia earthquakes since January 1969. An important feature available in HYPOELLIPSE is the calculation of confidence ellipsolds for each hypocenter. The ellipsoids provide valuable insight into the effect of network geometry on possible hypocentral errors. 


\section{VELOCITY MODELS}

Our experlence with locating earthquakes in southern Alaska suggests that significant lateral variations are present in the velocity structure across the network. Such variations might be expected by considering the complicated geology and tectonics of the region (eg., Plafker, 1967). Very little information in the form of direct measurement is available for the velocity structure in southern Alaska. In previous catalogs, only two P-wave velocity models consisting of horizontal layers of constant velocity were used to locate the earthquakes (eg. Stephens, and others, 1979). These velocity models were derived by minimizing the travel-time residuals for selected sets of earthquakes in the Cook Inlet region (Mode? $A$ of Matumoto and Page, 1969) and near Valdez. The models proved adequate for locating earthquakes as far east as Kayak Island, but earthquakes located farther to the east of ten had large travel-time residuals at nearby stations. An improved velocity model for the region east of Kayak Island was developed by minimizing the travel-time residuals for a selected set of aftershocks from the $1979 \mathrm{St}$. Elias earthquake that occurred north of I cy Bay (5tephens, and others, 1980). A significant difference between this model and the earlier ones is that it consists of a single layer of linearly increasing velocity over a half-space of constant velocity.

In the preparation of this catalog, the method of assigning velocity models to calculate theoretical travel-times to various stations is different from that used in some earlier catalogs. Previous $1 y$, the velocity model used was determined by the region in which the earthquake occurred and would then be the same for all stations for that event. In the revised procedure, each station always uses the same velocity model, and the model used is determined by the region in which the station is located. Thus, a station in the eastern region will use the eastern velocity model to calculate travel times from events that occur in the western, central and eastern parts of the network.

West of $148^{\circ} 45^{\prime}$ W the velocity model used is specified as follows:

\begin{tabular}{ccc} 
Layer & Depth $(\mathrm{km})$ & Pvelocity \\
\cline { 2 - 3 } 1 & $0-0$ & 2.75 \\
2 & $0-4$ & 5.3 \\
3 & $4-10$ & 5.6 \\
4 & $10-15$ & 6.2 \\
5 & $15-20$ & 6.9 \\
6 & $20-25$ & 7.4 \\
7 & $25-33$ & 7.7 \\
8 & $33-47$ & 7.9 \\
9 & $47-65$ & 8.1 \\
10 & below 65 & 8.3
\end{tabular}

The thickness, $D$, of the first layer is allowed to vary between stations to account for the presence of thick sections of low-velocity sediments beneath the stations NKA and NNL, which are located in the Cook Inlet basin. For these stations $D$ is $4 \mathrm{~km}$. For all other stations $D$ is $0.01 \mathrm{~km}$. It is recognized that a model comprised of uniform horizontal layers may be a poor representation of the actual velocity structure, particularly in the vicinity of a subduction zone (Mitronovas and Isacks, 1971; Jacob, 1972), a) though such 
a model does have the advantage of simplifying the computation of travel-times. In order to determine any bias that might result from this approximation, a set of events in the Berioff zone below Cook Inlet was relocated using a ray-tracing program of E. R. Engdahl that incorporates a more realistic, three-dimensional velocity model (Lahr, 1975). Hypocenter

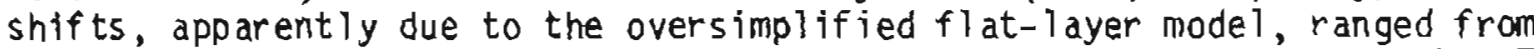
near zero at a depth of $60 \mathrm{~km}$ to as great as $25 \mathrm{~km}$ at the $160 \mathrm{~km}$ depth. The offsets were oriented in such a way that the dip of the Benloff zone would appear to be too great for locations based on a flat-layered model.

For earthquakes that occur between $148^{\circ} 45^{\prime} \mathrm{W}$ and $144^{\circ} 30^{\prime} \mathrm{W}$, the velocity model used to locate the events is specified by:

\begin{tabular}{ccc} 
Layer & Depth $(\mathrm{km})$ & Pvelocity $(\mathrm{km} / \mathrm{s})$ \\
\cline { 2 - 3 } & 0.0 & 2.75 \\
2 & 0.01 & 6.4 \\
3 & below 39 & 8.0
\end{tabular}

East of $144030^{\prime}$ the P-wave velocity of the layer increases linearly from $5.0 \mathrm{~km} / \mathrm{s}$ at the surface to $7.8 \mathrm{~km} / \mathrm{s}$ at $32 \mathrm{~km}$ depth, while the half-space has a velocity of $8.2 \mathrm{~km} / \mathrm{s}$.

P-phase travel-time delays are applied to stations in the network that have consistent and large residuals for the locations of large groups of earthquakes. Each station has three delays (DLYI, OLYZ and OLY3 of Table 1) assigned to it that correspond to the western, central, and eastern parts of the network. The particular delay that is used to locate an earthquake is determined by the region in which the earthquake occurs. For example, a station near Icy Bay that is used to locate an earthquake beneath Cook Inlet wi\}l be assigned a delay OLY1, but the same station will use DLY 3 to locate an earthquake that occurs beneath Icy Bay. Additional delays are applied at several statlons to correct for a satellite link in the relay of the signal. $\mathrm{S}$-phase delays are determined by multiplying the P-delay by 1.78 , the $\mathrm{P}$ to $\mathrm{S}$ velocity ratio.

The initial trial depths for earthquakes which occur in the western, central and eastern parts of the network are 75,30 and $15 \mathrm{~km}$, respectively, and refiect a progressive decrease in the range of depths of earthquakes from west to east. 


\section{MAGNI TUDE}

Magnitudes are determined from either the signal duration or the maximum trace amplitude. Eaton and others (1970) approximate the Richter local magnitude, whose definition is tied to maximum trace amplitudes recorded on standard horizonta? Wood-Anderson torsion seismographs, by an amplitude magnitude based on maximum trace amplitudes recorded on high-gain, highfrequency vertical seismographs such as those operated in the Alaskan network. The amplitude magnitude XMAG used in this catalog is based on the work of Eaton and his co-workers and is given by the expression (Lee and Lahr, 1972)

$$
X M A G=\log _{10} A-B_{1}+B_{2} \log _{10} D^{2}
$$

where $A$ is the equivalent maximum trace amplitude in millimeters on a standard Hood-Anderson seismograph, $D$ is the hypocentral distance in kilometers, and $\mathrm{B}_{1}$ and $\mathrm{B}_{2}$ are constants. Differences in the frequency response of the two seismograph systems are accounted for in $A$. It is assumed, however, that there is no systematic difference between the maximum horizontal ground motion and the maximum vertical motion. The terms $-B_{1}+B_{2} \log _{10} D^{2}$

approximate Richter's - $\log _{10} A_{0}$ function (Richter, 1958, $\mathrm{p}$. 342), which expresses the trace amplitude for an earthquake of magnitude zero as a function of epicentral distance.

For small, shallow earthquakes in central California, Lee and others (1972) express the duration magnitude FMAG at a given station by the relation

$$
\text { FMAG }=-0.87+2.00 \log _{10} T+0.0035 \mathrm{DEL}
$$

where $T$ is the signal duration in seconds from the $P$-wave onset to the point where the peak-to-peak trace amplitude on the Geotech Model $6585 \mathrm{film}$ viewer falls below $1 \mathrm{~cm}$ and DEL is the epicentral distance in kilometers.

Comparison of XMAG and FMAG estimates from equations (1) and (2) for 77 Alaskan shocks in the depth range 0 to $150 \mathrm{~km}$ and in the magnitude range 1.5 to 3.5 reveals a systematic linear decrease of FMAG relative to XMAG with increasing focal depth. To remove this discrepancy, a linear dependence on depth is added to the expression for FMAG as follows:

$$
F M A G=-1.15+2.00 \log _{10} T+0.007 Z+0.00350 E L
$$

where 2 is the focal depth in kilameters.

The magnitude preferentially assigned to each earthquake in this catalog is the FMAG estimate. The XMAG value is used only where no FMAG can be determined. 


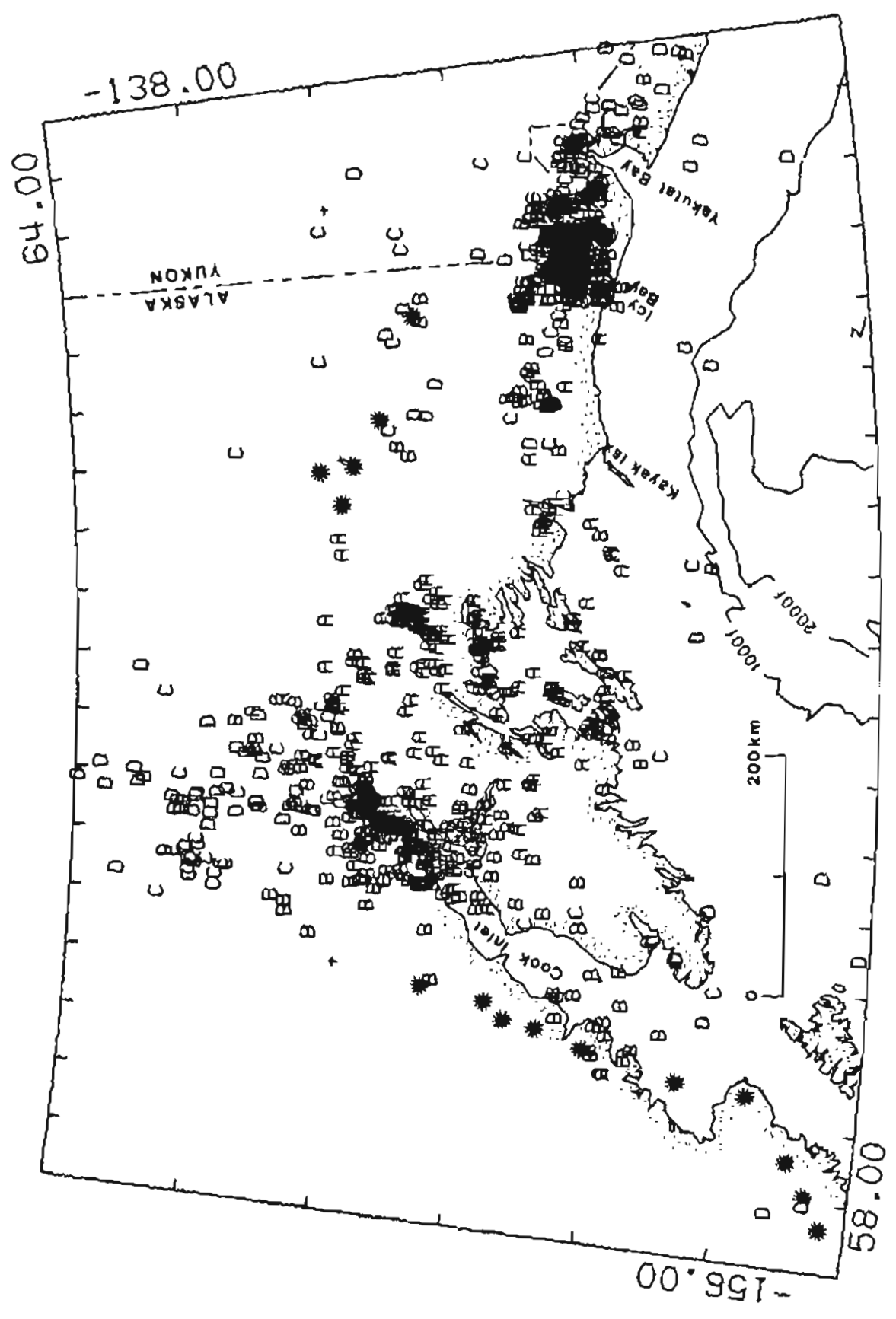

$a^{\frac{5}{4}}$

s. is

开苋

的

목 㟔

要

t

它要

-

d등.

$\infty$

펑

ᄃ

는

준완은

$1 \pm$

궁요

o

施要

舟

중 홍

究点

a

选点

s

造造

的

को

要

预乐过

웡잉

Q 氙先

的

궁회

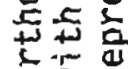

第等

号昰邑

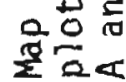

$\infty^{\circ}$

家 


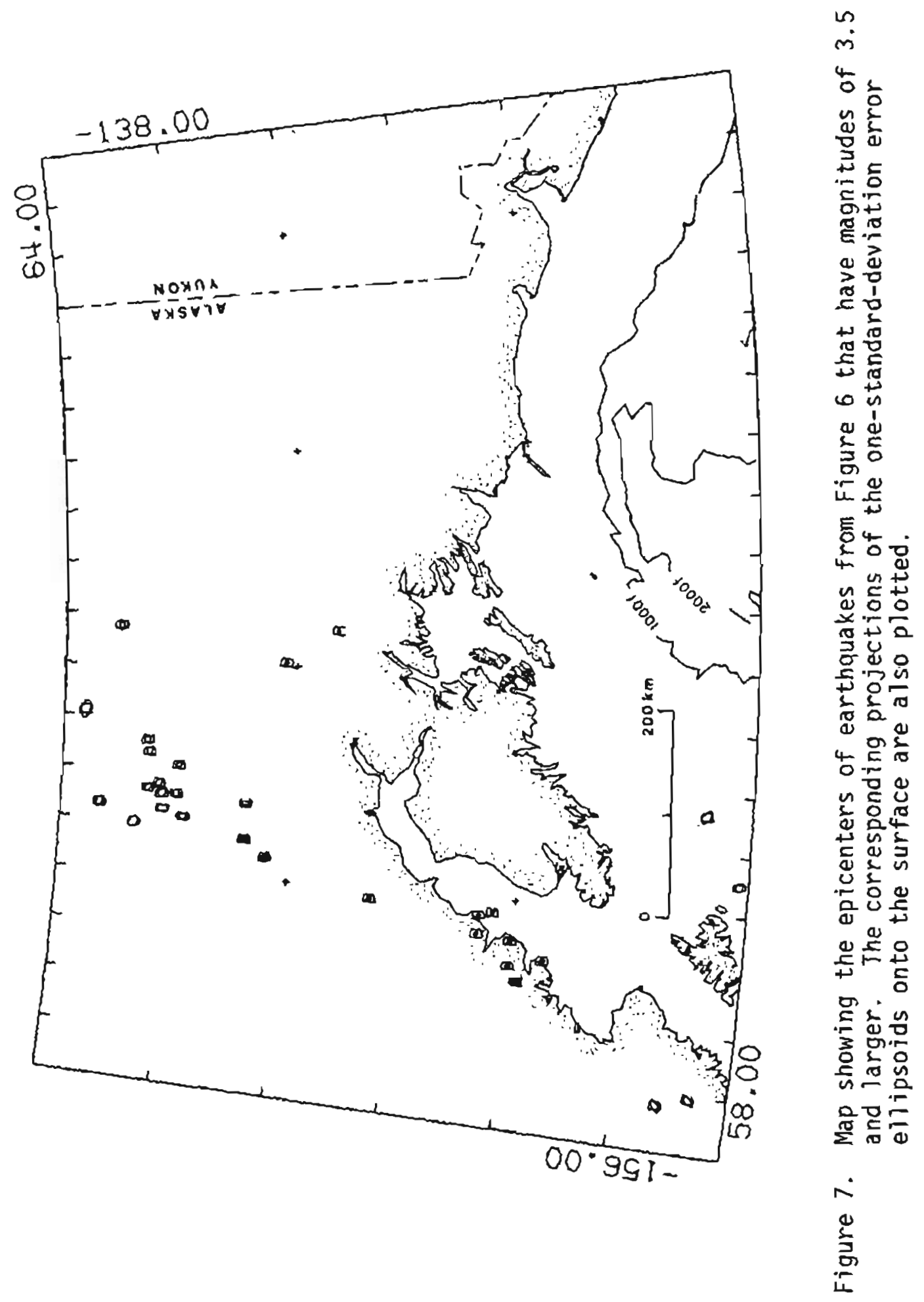




\section{ANALYSIS OF QUALITY}

Two types of errors enter into the determination of hypocenters: systematic errors limiting the accuracy of hypocenters and random errors limiting the precision. Systematic errors arise from an incorrect velocity mode $T$, misidentification of phases, or systematic timing errors and can be evaluated through controlled experiments such as locating the coordinates of a known explosion. Random errors result from random timing errors and are estimated for each earthquake through the use of standard statistical techniques.

For each earthquake, HYPOELLIPSE calculates the lengths and or ientations of the principal axes of the joint confidence ellipsoid. The one-standarddeviation confidence ellipsoid describes the region of space within which one is 68 percent confident that the hypocenter lies, assuming that the only source of error is random reading error. The ellipsoid is a function of the station geometry for each individual event, the velocity model assumed and the standard deviation of the random reading error. The standard deviation determined from repeated readings of the same phases by four seismologists is as small as 0.01 to $0.02 \mathrm{~s}$ for the most impulsive arrivals and as large as 0.10 to $0.20 \mathrm{~s}$ for emergent arrivals. The confidence ellipsoids are computed for a standard deviation of $0.16 \mathrm{~s}$ and therefore likely overestimate the $68 \%$ confidence regions. The standard deviation of the residuals for an individual solution is not used to calculate the confidence ellipsoid because it contains information not only about random reading errors but also about the incompatibility of the velocity model to the data. Thus, the confidence ellipsoid is a measure of the precision of the hypocentral solution. In a few extreme cases the value calculated for one of the ellipsoid axes becomes very 1 arge corresponding to a spatial direction with very great uncertainty. In these cases an upperbound length of $25 \mathrm{~km}$ is tabulated.

To fully evaluate the quality of a hypocenter one must consider both the confldence ellipsold and the root mean square (RMS) residual for the solution. The RMS residual reflects both systematic and random errors, but the random errors are typically much smaller. Hence the RMS residual is primarily a measure of the incompatibility of the velocity model, misinterpretation of phases and systematic timing errors. Interpretation of the RMS residual may depend upon the location of the earthquake. In areas where the velocity model is incompatible with the real earth, RMS residuals could be large and betray the incompatibllity; alternatively, the RMS residuals could be small and not reflect the error in a bad hypocenter. Where the velocity model is compatible, however, a large RMS residual would indicate probable misreadings of phases.

Other parameters provided by HYPOELLIPSE that are useful in evaluating the quality of a hypocentral solution are: GAP, the largest azimuthal separation between stations measured from the epicenter; 03, the epicentral distance of the third closest station; NP, the number of $P$ arrivals used in the solution; and NS, the number of $S$ arrivals used in the solution. If GAP exceeds $180^{\circ}$, the earthquake lies outside the network of avallable stations and the solution is generally less reliable than for events occurring inside the network. 


\section{DISCUSSION OF CATALOG}

Origin times, focal coordinates, magnitudes and related parameters for 1376 earthquakes from January-March 1980 are 1 isted in the Appendix. Epicenters for these shocks are plotted in Figure 6 . In Figure 7 , only the earthquakes with magnitudes greater than 3.5 are plotted. Vertical sections showing the depth distribution of all of the shocks are presented in Figures 8 and 9 .

We estimate that this catalog is reasonably complete for shocks larger than magnitude 3.5 in the western, 2.5 in the central, and 2.0 in the eastern regions of the area covered by the network. The minimum magnitude of the listed earthquakes ranges from 0.2 for shallow shocks to 2.3 for the deeper shocks.

The precision of the hypocenters or the relative accuracy of the locations of neighboring events is represented by the confidence ellipsoids. The precision of epicenters, expressed in terms of the maximum axes of the projected one-standard-deviation confidence ellipsoids (ERH), averages 4.3, 2.2 , and $2.3 \mathrm{~km}$, respectively, in the eastern, central, and western parts of the network. Similarly, the precision of focal depth (ERZ) averages about $5.5,3.4$, and $5.4 \mathrm{~km}$, respectively. The variation in the precision of hypocenter determination across the network is strongly influenced by differences in the station coverage in the different regions.

The absolute accuracy of the earthquake locations is difficuit to evaluate in the absence of known explosions. Hypocenter biases equal to and larger than the dimensions of the confidence elfipsoids are not unlixely from the oversimplffied velocity model assumed in the preparation of this catalog.

The dominant feature in the distribution of epicenters is the large number of aftershocks from the $1979 \mathrm{St}$. Elias earthquake in southeastern Alaska. All of the aftershocks were located at depths less than $30 \mathrm{~km}$, which is consistent with the depths found for aftershocks in the early part of the sequence (Stephens and others, 1980). It is interesting to note that the aftershocks plotted here appear to form spatial clusters similar to those observed in the early part of the sequence. All of the aftershocks have magnitudes below 3.5, as determined by comparing Figures 6 and 7 . However, the coda magnitudes for the aftershocks reported here are probably systematically low relative to other magnitude scales, as dyscussed by Stephens and others (1980).

The seismicity throughout the remainder of the network does not vary significantly from that described for previous quarters (stephens and others, 1979; Fogleman, and others, 1978; Lahr, and others, 1974). A well-defined Benioff zone dips to the northwest beneath the Cook Inlet region (Figure 9, sections $G-J$ ). The depth to the top of this zone varies from about $50 \mathrm{~km}$ beneath the western Kenal peninsula to about 115 beneath the active volcanoes west of Cook Inlet. The dip of the Benioff zone appears to increase from northeast to southwest, but the depth to the seismic zone beneath the active volcanoes--Augustine, Iliamna, Redoubt and Spurr-- is nearly constant at about $115 \mathrm{~km}$.

All of the seismic activity in the southern part of the network east of 
$1460 \mathrm{~W}$ occurs at depths less than about $35 \mathrm{~km}$. The number of larger magnitude earthquakes which occur in the east is considerabiy smaller than that in the western part of the network (Figure 7). Most of the seismic activity in the eastern part of the network appears to be concentrated beneath I cy Bay and northeast of Kayak IsI and.

The contents of the Appendix may be obtained in forms amenable to computer Input (punched cards or magnetic tape) by contacting the authors.

\section{ACKNOWLEDGEMENTS}

We thank Robert Eppley, Wayne Jorgensen and the entire staff of the NOAA Tsunami Warning Center for their assistance in maintaining our recording equipment in Palmer. Alaska, as well as making their seismic data available to us.

We also wish to thank Hans Pulpan of the Geophysical Instytute of the University of Alaska for a cooperative operation of southern Cook Inlet seismograph stations.

We are indebted to all of those who have spent time fabricating, installing, and malntaining the seismograph network in Aldska, particularly John Roger, Greg Condrotte and Willi an Wong.

Betty McIntire and the staff of the USGS Anchorage office has been of great assistance in solving logistic problems, both in the field and in the office.

This catalog is patterned after those prepared for central Cal ifornia and we gratefully acknowledge Ors. W. H. K. Lee and R. L. Wesson for development of many of the procedures and techniques used herein.

This study was supported jointly by the U.S. Geological Survey and by the National Oceanic and Atmospheric Administration, under which a multi-year program responding to needs of petroleum development of the Alaskan continental she if is managed by the Outer Continental She if Environmental Assessment Program (OCSEAP) Office. 


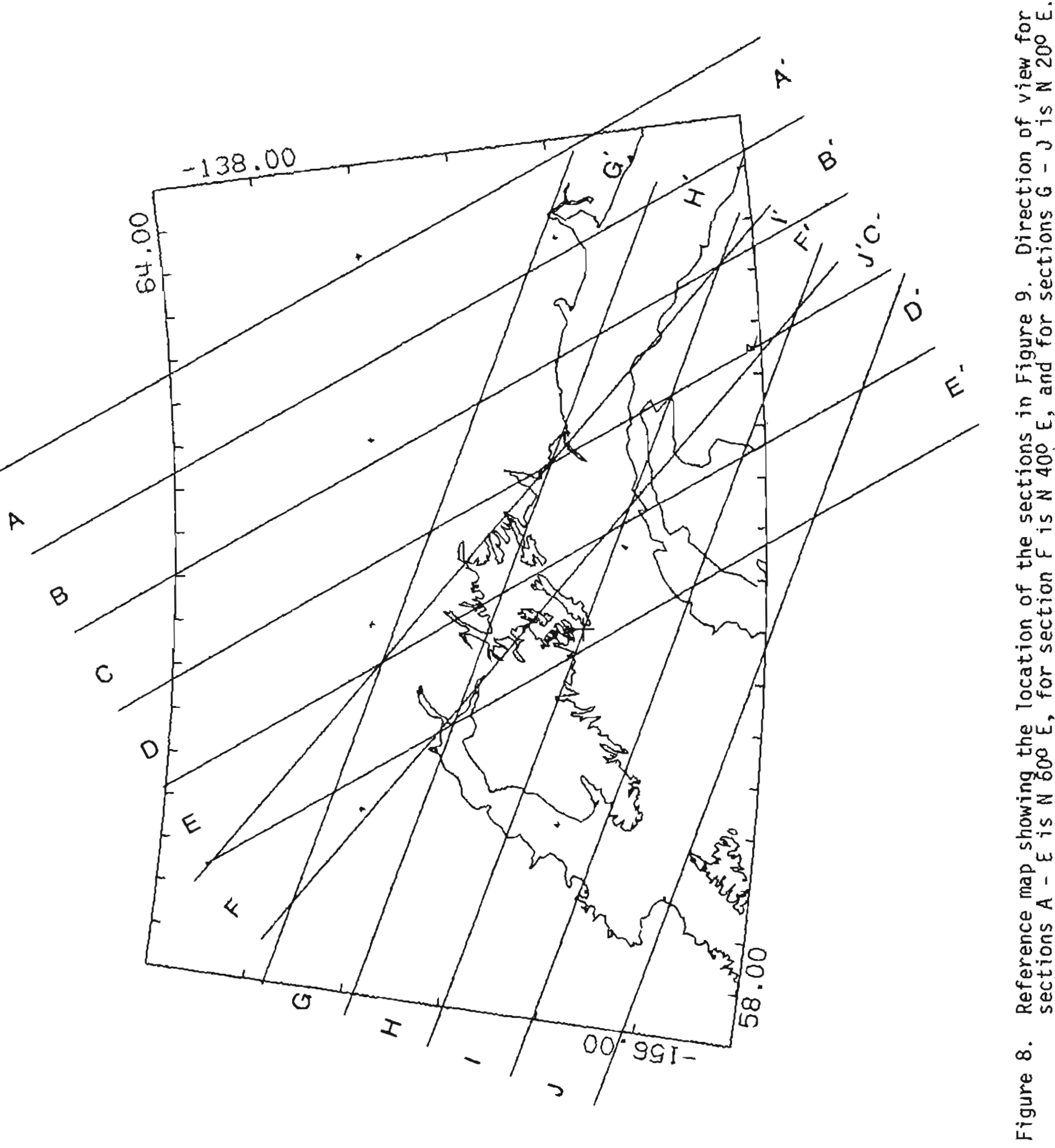




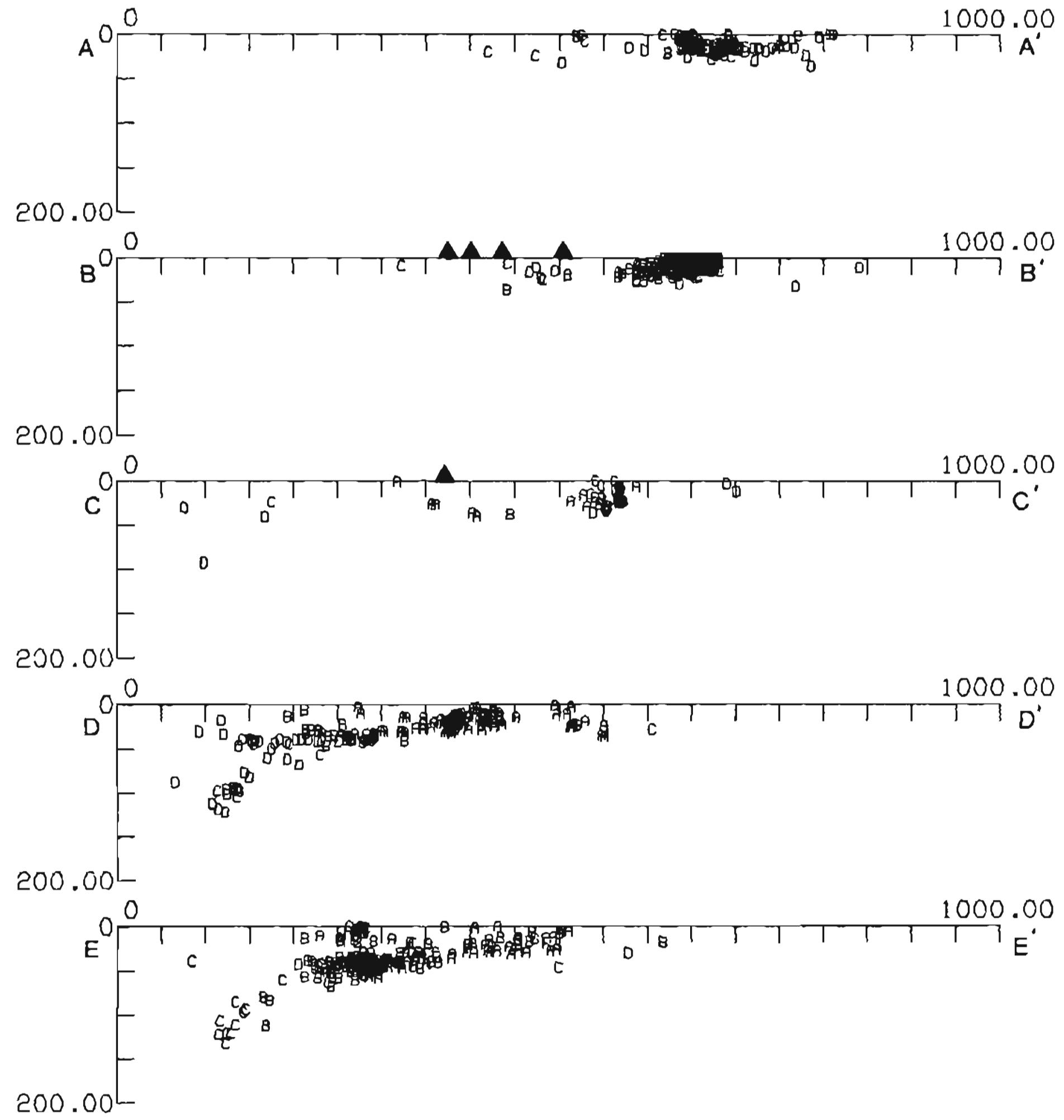

Figure 9. Vertical sections of hypocenters for the areas indicated in Figure 8. Quaternary volcanoes are plotted as triangles at zero depth. All distances are in kilometers. No vertical exaggeration. 


\section{REFERENCES}

Eaton, J. P., M. E. O'Neill, and 3. N. Murdock (1970). Aftershocks of the 1966 Parkfield-Cholame, California, earthquake: a detailed study, Bulletin Seismological Society of America 60, 1151-1197.

Fogleman, K., C. Stephens, J. C. Lahr, S. Helton, and M. Allan (1978). Catalog of earthquakes in southern Alaska, October-December 1977, U.S. Geological Survey, Open-F lle Report 78-1097, 28 p.

Jacob, K. H. (1972). Global tectonic implications of anomalous seismic $P$ traveltimes from the nuclear exploston Longshot, Journal of Geophysica? Research 77, 2556-2573.

King, P.B., compller (1969). Tectonic Map of North America, U.S. Geological Survey, scale $1: 5,000,000$.

Lahr, J. C. (1975). Detailed seismic investigation of Pacjfic-North American plate interaction in southern Alaska, Ph.D. dissertation, Columbia University, $141 \mathrm{p}$.

Lahr, J. C., E. R. Engdahl, and R. A. Page (1974). Locations and focal mechanisms of intermediate depth earthquakes below Cook inlet, Alaska, EOS $55,349$.

Lahr, J. C., 1980 HYPOELLIPSE/MULTICS: A computer program for determining local earthquake hypocentral parameters, magnitude, and first motion pattern, U.S. Geological Survey Open-File Report $80-59,59 \mathrm{pp}$.

Lee, W. H. X., and J.C. Lahr (1972). HYP071: a computer program for determining hypocenter, magnitude, and first motion pattern of local earthquakes, U.S. Geological Survey, Open-File Report, 100 p.

Lee, W. H. K., R. E. Bennett, and K. L. Meagher (1972). A method of estimating magnitude of local earthquakes from signal duration, U.S. Geological Survey, Open-File Report, $28 \mathrm{p}$.

Matumoto, T., and R. A. Page (1969). Microaftershocks following the Alaska earthquake of 28 March 1964: "Determination of hypocenters and crustas velocities in the Kenai Peninsula-Prince William Sound area", The Prince Willian Sourd, Alaska, Earthquake of 1964 and Aftershocks, vol, $2 B$ \& $C$, U. S. Coast and Geodetic Survey Publication 10-3, W.S. Govt. Printing office, Washington, 157-173.

Meyers, H. (1976). A historical summary of earthquake epicenters in and near Alaska, NOAA Technical Menorandum EDS NGSDC-1, 570.

Mitronovas, W., and B. L. Isacks (1971). Seismic velocity anomalies in the upper mantle beneath the Tonga-Kermadec island arc. Journal of Geophys 1cal Research, 76, 7154-7180.

Plafker, G. (1967). Geologic map of the Gulf of Alaska Tertiary Province, Alaska, U.S. Geological Survey Miscellaneous Investigations Map I-84, scale $1: 500,000$.

Richter, C.F. (1958). Elementary Seismology, W. H. Freeman and Co., San Francisco, 768 pp.

Rogers, J. A., S. Maslak, and J. C. Lahr (1980). A seismic electronic system with autonatic calibration and crystal reference, U.S. Geological Survey Open-F ti e Report 80-324, 130 p.

Stephens, C. D., J.C. Lahr, K. A. Fogleman, M. A. All an, and S. M. Helton (1979). Catalog of earthquakes in southern Alaska, January-March 1978, U.S. Geological Survey Open-File Report 79-718, 31p.

Stephens, C. D., J.C. Lahr, K. A. Fogleman, and R. B. Horner (1980). The St. Elías, Alaska earthquake of 28 February 1979: regional recording of aftershocks and short term pre-earthquake seismicity, Bulletin Seismological Society of America, 70, 1607-1633. 


\section{APPENOIX}

\section{Catalog of Earthquakes}

Earthquakes from southern Alaska are listed in chronological order. The following data are given for each event:

(1) Origin time in Universal Time (UT): date, hour (HR), minute (MN), and second (SEC). To convert to Alaska Standard Time (AST) subtract ten hours.

(2) Epicenter in degrees and minutes of north latitude (LAT N) and west longitude (LONG W).

(3) DEPTH, depth of focus in kilameters.

(4) MAG, duration magnitude (FMAG) of the earthquake, if available, otherwise amplitude magnitude (XMAG, Indicated by "a").

(5) NP, number of $P$ arrivals used in locating earthquake.

(6) NS, number of $S$ arrivals used in locating earthquake.

(7) GAP, largest azimuthal separation in degrees between stations.

(8) 03 , eptcentral distance in kilometers to the third closest station to the epicenter.

(9) RMS, root-mean-square error in seconds of the traveltime residuals:

$$
R M S=\sqrt{\sum_{i}\left(R_{P_{i}}^{2}+R_{S_{j}}^{2}\right) /(N P+N S)}
$$

where $R_{p}$ and $R_{S_{j}}$ are the observed minus the computed arrival times of $p$ - and $d_{-w a v e s}$ respectively at the $j-t h$ station.

(10) ERH, largest horizontal deviation in xilometers from the hypocenter within the one-standard-deviation confidence ellipsold. This quantity is a measure of the eplcentral precision for an event. Values of ERH that exceed $25 \mathrm{~km}$ are tabulated as $25 \mathrm{~km}$.

\section{Projection of ellipsoid} onto horizontal plane:

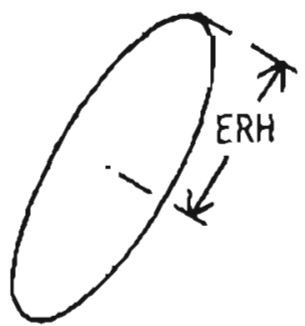


(11) ERZ, largest vertical deviation in kilometers from the hypocenter within the one-standard-deviation confidence ellipsoid. This quantity is a measure of the depth precision for an event. Values of ERZ that exceed $25 \mathrm{~km}$ are tabulated as $25 \mathrm{~km}$.

Projection of ellipsoid onto vertical plane:

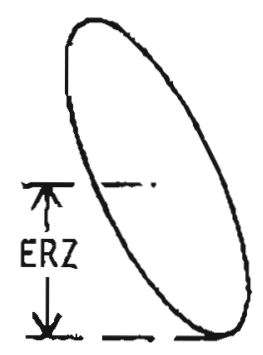

(12) Q, quality of the hypocenter. This index is a measure of the precision of the hypocenter (see section Analysis of Quality) and is calculated from ERH and ERZ as follows:

$$
\begin{array}{lll}
Q & \leq \frac{E R H}{2.5} & \leq \frac{E R Z}{2.5} \\
A & \leq 5.0 & \leq 5.0 \\
B & \leq 5.0 & \leq 10.0 \\
C & \leq 10.0 & >10.0
\end{array}
$$

(13) AZ1, OIP1, and SEI are the azimuth in degrees (clockwise from north), dip in degrees, and standard error in. kilometers of the most nearly horizontal of the three orincipal axes of the one-standarddeviation error ellipsoid. Values of SE1 that exceed $25 \mathrm{~km}$ are tabulated as $25 \mathrm{~km}$.

(14) AZ2, DIP2, and SE2 are defined as above, but correspond to the principal axis of intermediate dip.

(15) AZ3, DIP3, and SE3 are defined as above, but correspond to the most nearly vertical princtpal axis.

Magnitudes and felt reports listed below an event were obtained from the Preliminary Determination of Eplcenters of the USGS National Earthquake Information Service (NEIS), or the Department of Energy, Mines and Resources, $\mathrm{Canada}$ (EMR). The body-wave (mb) and surface-wave (MS) magnitudes are those determined by the NEIS.

A letter code after the depth indicates as follows:

C - Solution was constrained based on EMRC source.

D - Depth was constrained by a geophysicist.

P - Solution was constrained based on POE source.

W - Station weighting modified (for events outside of network). 
DRIGIN TIME LATN LONOH DEPTH MAG NF NS OAP DJ RMS ERH ERI O AII DLPI SEI AZZ DIFZ SER AZ3 DIP3 GEJ

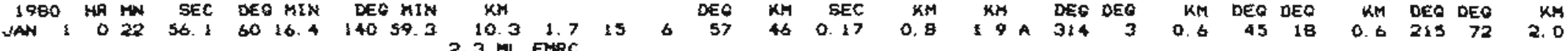

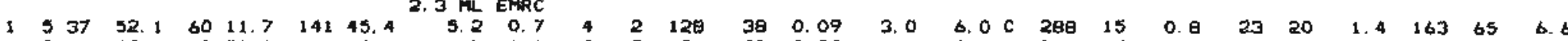

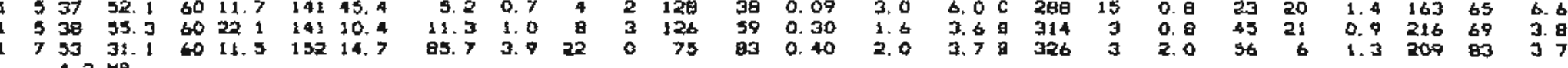

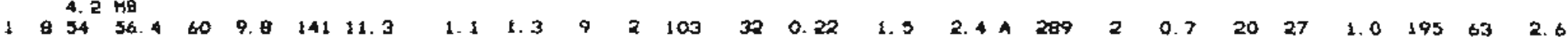

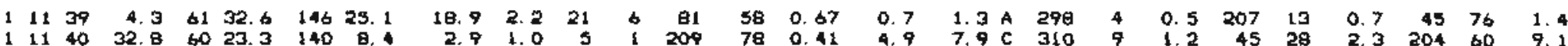

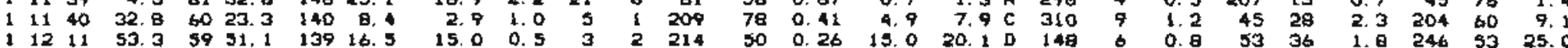

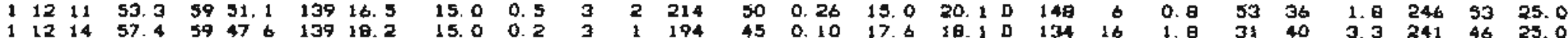

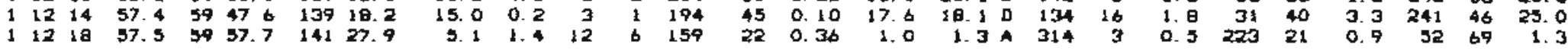

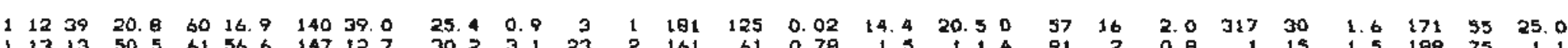

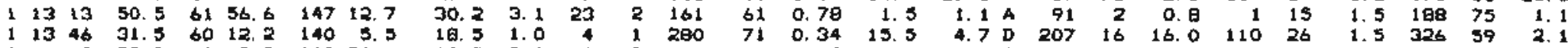

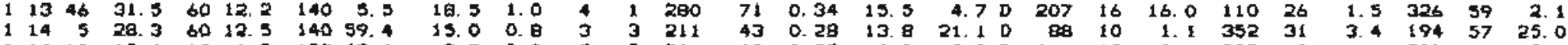

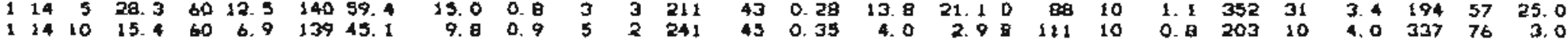

1143919.8 so $13,6 \quad 14039$ o. 0.952149

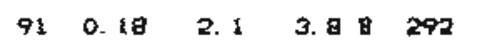
117370.4 to $41.9 \quad 143 \quad 9.3$

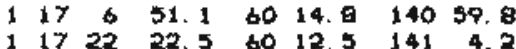

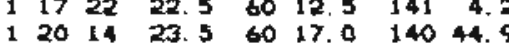

$12318 \quad 6.4 \quad 6021.6 \quad 14041$. 1.6 $182 \quad 69 \quad 4.0$ $\begin{array}{rrrrrrrr}2 & 3 & 36 & 50.4 & 60 & 13.6 & 146 & 56.3 \\ 2 & 4 & 0 & 50.7 & 30 & 45.2 & 149 & 46.3\end{array}$ $\begin{array}{llllllll}2 & 6 & 14 & 47.0 & 59 & 56.0 & 152 & 50.2 \\ 2 & 6 & 32 & 29.0 & 61 & 1.0 & 149 & 34.6\end{array}$ $\begin{array}{lllllllll}2 & 6 & 30 & 29.9 & 61 & 1.9 & 149 & 34.6 \\ 2 & 7 & 58 & 33.4 & 60 & 0.3 & 140 & 34.9\end{array}$ 2 日 20 40.1 41 13. 9 146 31. $\begin{array}{rrrrr}12.4 & 2.3 & 20 & 7 & 12 \\ 41.5 & 1.5 & 6 & 7 & 8\end{array}$ 64 0. 15 $\begin{array}{rrrrrrr}1.3 & 100 & 4 & 1.4 & 294 & 66 & 3.1\end{array}$ $\begin{array}{lllllll}1.1 & 10 & 39 & 1.3 & 180 & 52 & 5.6\end{array}$ $0.6 \quad 147 \quad 82 \quad 2.0$ 917 7. 6021.0 140 40.

$\begin{array}{rrrrr}97.9 & 3.4 & 20 & 3 & 95 \\ 47.3 & 1.6 & 9 & 7 & 90\end{array}$ 15.5 2. 1 11 4 135 $\begin{array}{rrrrr}14.0 & 2.0 & 22 & 10 & 60 \\ 1.3 & 1.9 & 21 & 7 & 35\end{array}$ 56 o. 32

21055 38. 8 60 20. 1 141 37.6

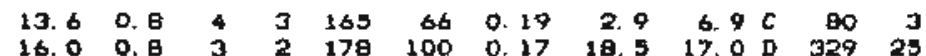

$\begin{array}{rrrrrrr}2.2 & 349 & 22 & 0.7 & 177 & 69 & 7.1 \\ 0.9 & 80 & 37 & 2.7 & 214 & 43 & 25.0\end{array}$

2 16 3553.3 60 17.0 141 16.

$219 \quad 13 \quad 52.4$ 59 $59.9 \quad 14131.9$

2231736.0 61 42. 2150 8. $\begin{array}{llllllll}3 & 0 & 16 & 38.0 & 60 & 17.1 & 141 & 3.0\end{array}$ $\begin{array}{llllllll}3 & 1 & 10 & 2 . & 59 & 12.3 & 142 & 50 .\end{array}$ $\begin{array}{llllllll}3 & 2 & 24 & 14.4 & 39 & 23.5 & 142 & 39.2 \\ 3 & 6 & 45 & 31.9 & 60 & 11.4 & 140 & 37.0\end{array}$ $3724 \quad 33.0 \quad 5934.4 \quad 13911.2$ $\begin{array}{llllllll}3 & 11 & 37 & 19.3 & 60 & 21.3 & 140 & 19.4\end{array}$ $313714.0 \quad 4220.4 \quad 149 \quad 8.3$

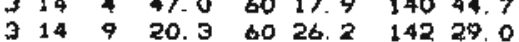

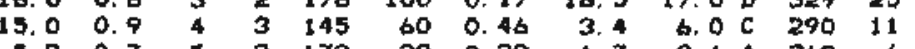

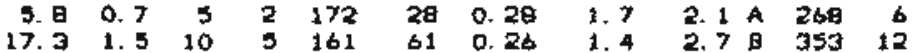
\begin{tabular}{rrrrrrrr}
0.7 & 17 & 74 & 1.3 & 7 & 55 & 2.9 \\
\hline 12 & 07 & 1.1 & 230 & 69 & 2.9
\end{tabular}

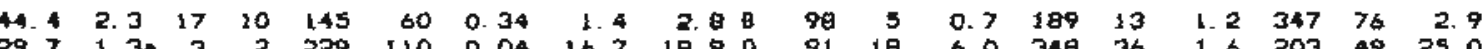

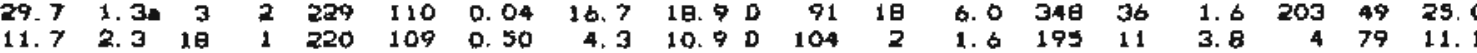

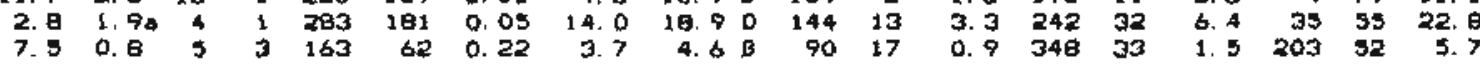

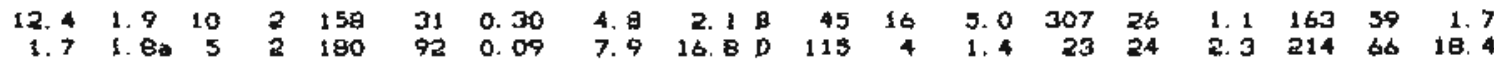

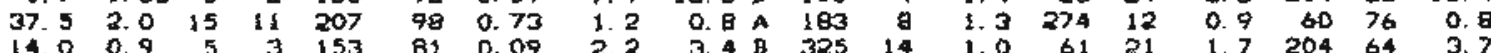

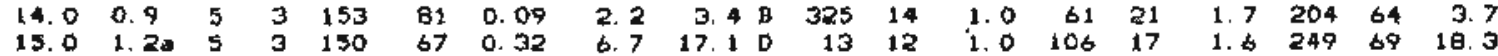


SOUTHERN ALABKA EAGTHQUANES, JAN - MAR 1980

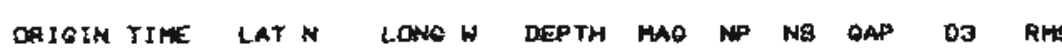

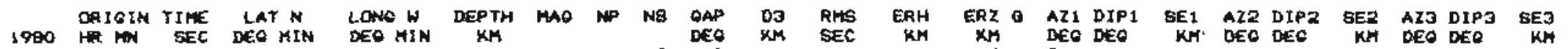

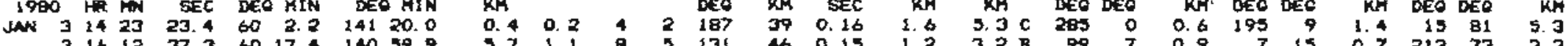

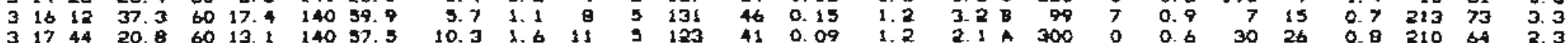

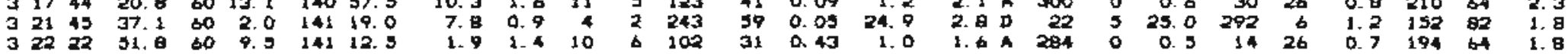

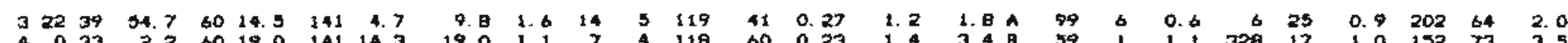

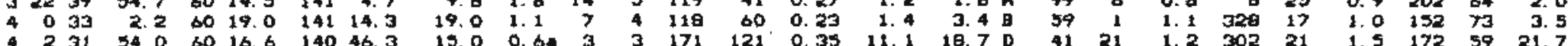

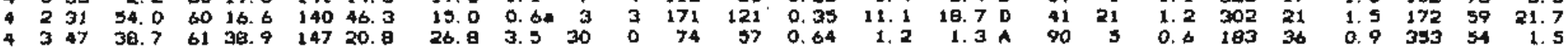
3. 7 MO 4359 10.3 \$1 39. 4.47 20.4

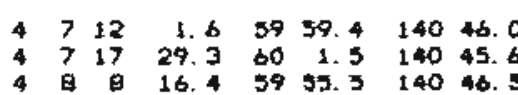
$\begin{array}{cccccccc}4 & 9 & 16.4 & 59 & 53.5 & 140 & 46 . \\ 4 & 93 & 37.1 & 59 & 97.0 & 140 & 46 .\end{array}$ $\begin{array}{rrrrrrr}4 & 93 & 37.1 & 59 & 57.0 & 140 & 46.6 \\ 4 & 9 & 32.1 & 59 & 55.4 & 140 & 46.3\end{array}$ $\begin{array}{rrrrrrrr}4 & 9 & 1 & 18.3 & 59 & 53.8 & 140 & 49.1 \\ 4 & 9 & 15 & 23.3 & 00 & 11.0 & 141 & 3.7\end{array}$ 4947 8. 1 bo 14.814017 .0

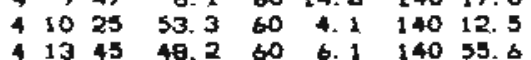

$41412 \quad 29.0$ b0 15. 114039.4 $41417 \quad 40.4$ bo $14.6 \quad 14042.6$

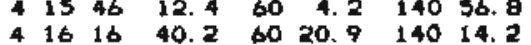

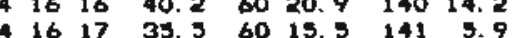
29.03 .019 FELT AT ANCHOHAOE 0.66

\begin{tabular}{|c|c|c|c|c|c|c|}
\hline $\begin{array}{l}\text { G. } 1 \\
0.3 \\
0.2 \\
3.6 \\
0.9\end{array}$ & $\begin{array}{l}1.6 \\
2.0 \\
0.9 \\
1.2 \\
0.9\end{array}$ & $\begin{array}{r}11 \\
16 \\
5 \\
5 \\
5\end{array}$ & $\begin{array}{l}6 \\
1 \\
2 \\
3 \\
3\end{array}$ & $\begin{array}{l}129 \\
121 \\
192 \\
191 \\
192\end{array}$ & $\begin{array}{l}31 \\
31 \\
46 \\
44 \\
46\end{array}$ & $\begin{array}{l}0.26 \\
0.30 \\
0.12 \\
0.20 \\
0.21\end{array}$ \\
\hline & 1. 2 & 6 & 3 & 200 & 46 & 0.47 \\
\hline 10. 1 & 1.4 & i1 & 日 & 112 & 35 & 0.23 \\
\hline $\begin{array}{l}10.2 \\
10.3\end{array}$ & $\begin{array}{l}0.7 \\
1.4\end{array}$ & 宣 & $\frac{2}{7}$ & $\begin{array}{l}104 \\
143\end{array}$ & $\begin{array}{l}91 \\
91\end{array}$ & $\begin{array}{l}0.15 \\
0.49\end{array}$ \\
\hline 10.6 & 1. 2 & 9 & 4 & 128 & 31 & 0. 20 \\
\hline
\end{tabular}

97

O. 18740

1.2 b 50 1.8

$\begin{array}{rrrrrrr}11.3 & 0.8 & 4 & 2 & 179 & 96 & 0.05 \\ 13.1 & 0.5 & 4 & 2 & 175 & 100 & 0.02 \\ 10.4 & 0.1 & 4 & 1 & 147 & 96 & 0.13\end{array}$

$\begin{array}{rrrrrrr}10.4 & 0.1 & 4 & 1 & 147 & 90 & 0.13 \\ 3.1 & 0.7 & 4 & 1 & 199 & 126 & 0.29\end{array}$

$\begin{array}{rrrrrrr}416 & 26 & 24.9 & 63 & 4.9 & 150 & 0.8 \\ 417 & 0 & 31.6 & 60 & 9.9 & 141 & 39.6\end{array}$

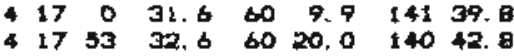

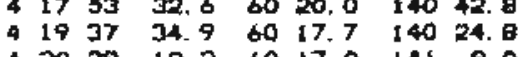

$104,3,3$

$\begin{array}{llll}1.3 & 1.8 & \text { A } & \text { Bb } \\ 1.3 & 1.9 A & 277 \\ 2.1 & 3.3 & \text { E } & 289\end{array}$

0.6 $354 \quad 18$

$1.219472 \quad 1.9$

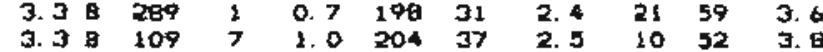

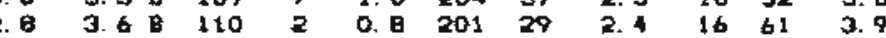

4203918.26017 .0 iी 8.0

$\begin{array}{rrrrrr}0.7 & 5 & 1 & 129 & 170 & 0.44 \\ 0.72 & 0.14\end{array}$

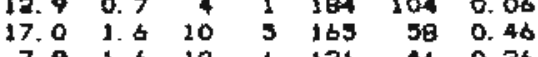

$50.046 .96015 .5 \quad 14115.9$ $=10$ 3e. 1 59 37. 139 36. 3 $\begin{array}{rrrrrrrr}5 & 1 & 42 & 17.0 & 60 & 10.3 & 141 & 1.9 \\ 3 & 2 & 47 & 47.5 & 60 & 5.4 & 140 & 44.6\end{array}$ 460.36

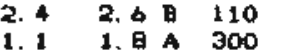

2. $3 \quad 472 \quad 2.7$ $\begin{array}{lllllllll}3.0 & 0.6 & 30 & 28 & 0.7 & 210 & 62 & 2.0\end{array}$

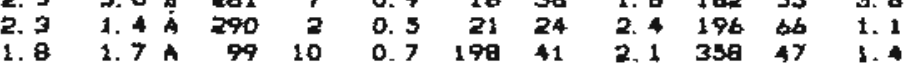

$5395 \quad 9.4 \quad 6020.7 \quad 141 \quad 11.6$ 5347 46.0 5020.9 141 24.

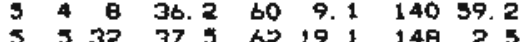
$\begin{array}{llllllll}5 & 5 & 32 & 37.5 & 62 & 19.1 & 148 & 2.5 \\ 3 & 7 & 23 & 45.3 & 60 & 12.9 & 141 & 4.2\end{array}$ $51210 \quad 9.6 \quad 6034.7 \quad 14131.3$ $\begin{array}{lllllll}12 & 37 & 5.2 & 60 & 17.2 & 141 & 10.8\end{array}$ $\begin{array}{lllllllll}5 & 13 & 6 & 35.0 & 60 & 9.3 & 141 & 20.3\end{array}$

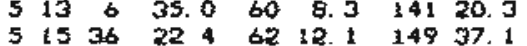

$\begin{array}{lllll}4.0 & 0.7 & 3 & 4 & 142 \\ 2.3 & 0.3 & 4 & 3 & 149\end{array}$

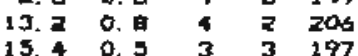
$\begin{array}{lllll}1.7 .7 & 0.9 & 3 & 3 & 297 \\ 1 & 8 & 5 & 139\end{array}$

9. $7 \quad 1.2 \quad 10 \quad 6 \quad 123$ $\begin{array}{ccccc}0.3 & 12 & 9 & 96\end{array}$ $\begin{array}{rrrrr}40.3 & 2.1 & 25 & 5 & 104 \\ 10.9 & 0.9 & 7 & 5 & 147\end{array}$ $\begin{array}{rllll}75.0 & 1.2 & 9 & 8 & 110 \\ 7.3 & 0.7 & 7 & 5 & 150\end{array}$

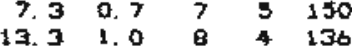
$\begin{array}{lllll}13.3 & 1.0 & 9 & 4 & 136 \\ 16.5 & 0.6 & 4 & 1 & 153\end{array}$

\subsection{8}

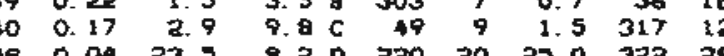

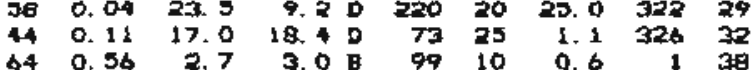

$58 \quad 0.40$ 75 0. 35

1. O 1.4A 291 $99 \begin{array}{llllllll}55 & 2.0 & 9.4 \mathrm{C} & 94 & 3 & 0.6 & 315 & 39\end{array}$ $\begin{array}{llllllllllllll}62 & 0.43 & 1.0 & 2.4 & \text { A } & 212 & 5 & 1.0 & 121 & 10 & 0.8 & 320 & 79 & 2.5 \\ 35 & 0.11 & 1.8 & 3.3 & 0 & 314 & 12 & 0.6 & 50 & 24 & 0.6 & 200 & 63 & 3.7\end{array}$

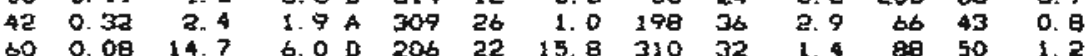

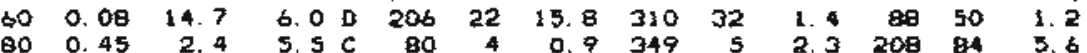


OP IOIN TIME LAT N
HR MN SONG WI JNA 3 is 37 11.9 62 B.9 147 17.6 $\$ 10931.25955 .414047 .2$

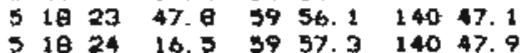

DEPTH HAQ NP NS GAF MM MA GAF DJ RMS 30. 2.2 it 4207 102 0.79

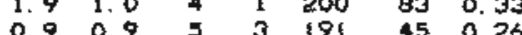
7.8 2.2 $17 \quad 4133$

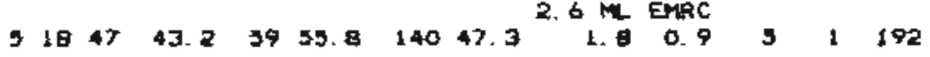
340.46 450.08 51046 40. 3 60 $15.9 \quad 14111.7$

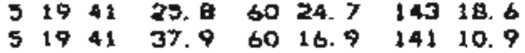
5 2010 103.3 59 55.5 140 47. a. $4 \quad 1,2 \quad 9 \quad 4 \quad 146$ $52010 \quad 43.35955 .514047 .2$ 5. 7 0. 7

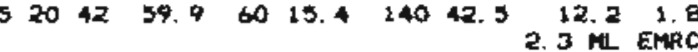

530.17 s) 0.06

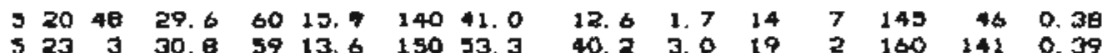
04759.75957 .314116 .0 $6 \quad 1 \quad 0 \quad 56.8$ 60 $18.3 \quad 140 \quad 39$.

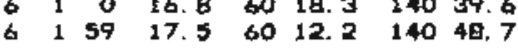

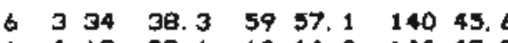
$\begin{array}{rrrrrrr}5.9 & 0.5 & 3 & 2 & 195 & 123 & 0.02 \\ 12.6 & 0.9 & 6 & 2 & 153 & 71 & 0.03\end{array}$

ERH ERI O AZI DIPI SE1 AI2 OIPZ SEZ AZ3 DIP3 SE3

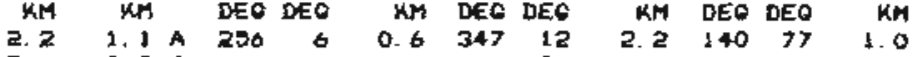

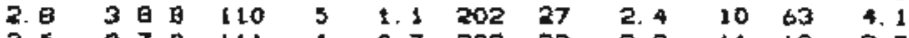

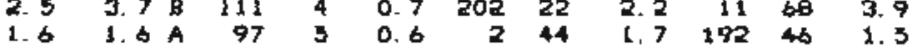

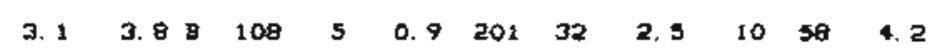
41392.6 5014.0 14040.3

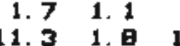
2. 4 M EMAC

$6450 \quad 14.3 \quad 60 \quad 15.9 \quad 140 \quad 36.6$ $\begin{array}{llllllll}6 & 4 & 54 & 58.3 & 60 & 46.3 & 140 & 56.4 \\ 6 & 5 & 5 & 56.0 & 62 & 30.1 & 149 & 36.3\end{array}$

9. 1.613 \& 131.420 .13 $\begin{array}{llllllll}6 & 5 & 24 & 35.9 & 61 & 44.0 & 149 & 44.1 \\ 6 & 11 & 10 & 15.6 & 60 & 2 \% .4 & 141 & 21.0\end{array}$ 611 21 17.2 60 $30.1 \quad 145 \quad 4.7$

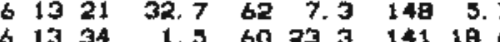

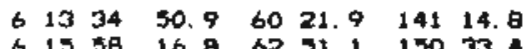

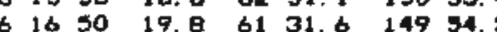
6 $1919 \quad 37.5$ 61 $2.2146 \quad 16.2$ $62076.56020 .0 \quad 141 \quad 13.4$

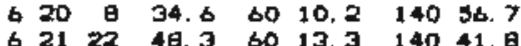

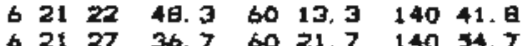
$62127 \quad 36.7 \quad 6021.7 \quad 140 \quad 54.7$

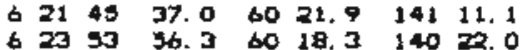

702345.5 10 17.9 146 33.6 $7229 \quad 9.16074 .5 \quad 14246.6$ $7 \quad 12 \quad 49.6 \quad 6016.514046 .7$ $7+2659.46016 .214055 .6$ $76+36.55957 .2 \quad 14118.6$ 7 (1) 18.4 59 37.5 141 13. 7749 1.6 59 59.6 140 12.

\subsection{9}

$3.4 \begin{array}{rrrr}1.0 & 4 & 3 & 169\end{array}$

$\begin{array}{rrrrr}32.3 & 1.3 & 13 & 4 & 137 \\ 2.3 & 17 & 5 & 196\end{array}$

$\begin{array}{llllllll}11.7 & 0.7 & 3 & 2 & 207 & 96 & 0.10\end{array}$ $\begin{array}{lllllll}93.3 & 3.0 & 14 & 3 & 119 & 199 & 0.39\end{array}$ $\begin{array}{lllllll}1.9 & 13 & 4 & 66 & 42 & 0.22\end{array}$ 17.0 0.8 5 I 154 5 5. 0.10 $\begin{array}{rrrrrrr}\text { 2. } 1 & 2.08 & 97 & 8 & 0.9 & 1 & 35 \\ 2.0 & 3.28 & 75 & 15 & 0.9 & 337 & 26\end{array}$

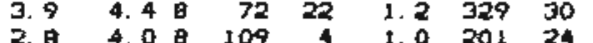

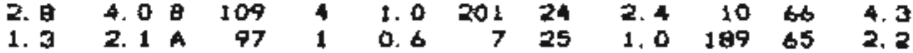

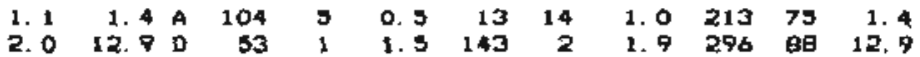

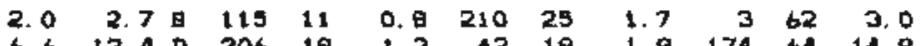

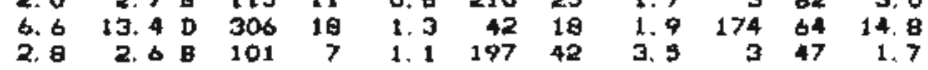
$9.60 .6 \quad 3 \quad 3 \quad 147$ 7.90 .7 $77 \quad 0.17 \quad 4.6$

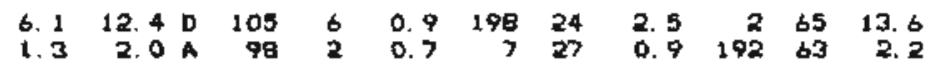

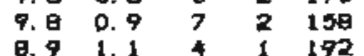

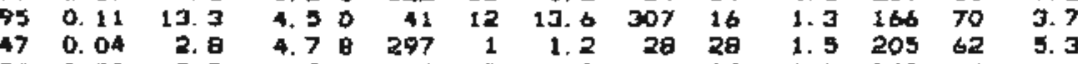

1.O 2. O A $332 \quad 12$

$\begin{array}{lllllll}0.7 & 66 & 15 & 0.6 & 205 & 70 & 2.1\end{array}$

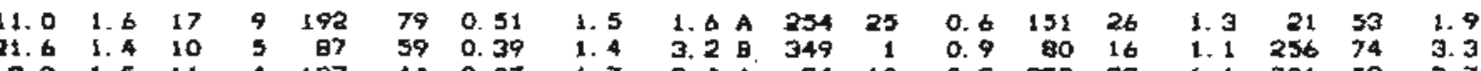

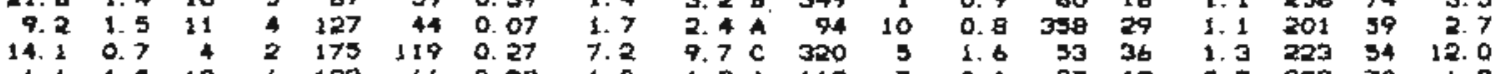
b. 1.512 a 172 0.27

5. 0 1. 4 11 4 123

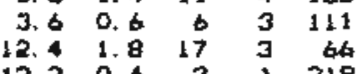
23 0. 32 1.0 $59.76 \quad 1.0 \quad 2.5$. 170 . 3

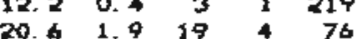

$\begin{array}{lllllllll} & 0.77 & 19.2 & 19.9 & 125 & 17 & 0.7 & 00 & 11\end{array}$

$1.1 \quad 17469 \quad 1.3$ o. 2757928 $2.41153 \quad 250$ 
SOUTHERN ALASHA EARTHOUAKES. JAN - MAR 1990

\begin{tabular}{|c|c|c|c|c|c|c|c|c|c|c|c|c|c|c|c|c|c|c|c|c|c|c|c|c|c|c|}
\hline 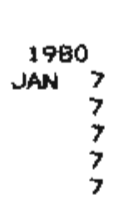 & $\begin{array}{l}\text { OR E } \\
\text { HR } \\
11 \\
12 \\
13 \\
13 \\
14\end{array}$ & $\begin{array}{l}10 I N \\
M N \\
27 \\
30 \\
1 \\
59 \\
28\end{array}$ & $\begin{array}{r}\text { TIME } \\
\text { SEC } \\
5.9 \\
19.9 \\
6.9 \\
7.9 \\
51.7\end{array}$ & $\begin{array}{l}\text { LA } \\
D E 0 \\
60 \\
60 \\
60 \\
60 \\
60\end{array}$ & $\begin{array}{l}A T N \\
\text { MIN } \\
4.6 \\
13.3 \\
20.8 \\
16.2 \\
16.1\end{array}$ & $\begin{array}{c}\text { LON } \\
\text { DEQ } \\
139 \\
141 \\
141 \\
140 \\
141\end{array}$ & $\begin{array}{r}\text { NG H } \\
\text { OMIN } \\
23.7 \\
4.9 \\
13.8 \\
48.4 \\
6.9\end{array}$ & $\begin{array}{c}\text { DEPTH } \\
\text { KM } \\
16.4 \\
0.6 \\
4.4 \\
14.2 \\
7.7\end{array}$ & $\begin{array}{l}0.4 \\
0.7 \\
1.0 \\
0.6 \\
1.2\end{array}$ & $\begin{array}{l}4 \\
7 \\
6 \\
5 \\
7\end{array}$ & $\begin{array}{r}\text { NS } \\
2 \\
3 \\
2 \\
2 \\
3\end{array}$ & $\begin{array}{l}\text { DAP } \\
\text { DEO } \\
245 \\
144 \\
155 \\
163 \\
153\end{array}$ & $\begin{array}{l}\text { D3 } \\
\text { KH } \\
48 \\
18 \\
56 \\
74 \\
54\end{array}$ & $\begin{array}{r}\text { RME } \\
\text { EEC } \\
0.41 \\
0.25 \\
0.18 \\
0.15 \\
0.12\end{array}$ & $\begin{array}{l}\text { ERH } \\
\text { KA } \\
7.9 \\
1.1 \\
2.1 \\
3.1 \\
1.9\end{array}$ & $\begin{array}{l}\text { ERI } \\
\text { MMH } \\
5.9 \\
3.1 \\
4.0 \\
3.2 \\
3.0\end{array}$ & $\begin{array}{l}C \\
B \\
0 \\
0 \\
B\end{array}$ & $\begin{array}{r}M 21 \\
D E 0 \\
120 \\
281 \\
104 \\
294 \\
72\end{array}$ & $\begin{array}{c}\text { DIP } 1 \\
\text { DEO } \\
20 \\
0 \\
6 \\
11 \\
14\end{array}$ & $\begin{array}{l}\text { BE } 1 \\
\text { KA } \\
1.4 \\
0.8 \\
1.0 \\
0.9 \\
0.9\end{array}$ & $\begin{array}{r}\text { AZ2 } \\
\text { DEC } \\
225 \\
11 \\
11 \\
35 \\
335\end{array}$ & $\begin{array}{c}\text { DIP2 } \\
\text { DEA } \\
36 \\
14 \\
24 \\
42 \\
25\end{array}$ & $\begin{array}{l}\text { SES } \\
\text { KM } \\
9.6 \\
0.9 \\
1.1 \\
1.7 \\
1.0\end{array}$ & $\begin{array}{r}A Z 33 \\
D E C \\
7 \\
191 \\
207 \\
192 \\
196\end{array}$ & $\begin{array}{c}\text { DIP3 } \\
\text { DEQ } \\
47 \\
76 \\
65 \\
46 \\
61\end{array}$ & $\begin{array}{r}\text { ges } \\
\mathrm{KH} \\
2.3 \\
3.1 \\
4.3 \\
4.1 \\
3.5\end{array}$ \\
\hline $\begin{array}{l}7 \\
7 \\
7 \\
7 \\
7\end{array}$ & $\begin{array}{l}16 \\
17 \\
20 \\
21 \\
21\end{array}$ & $\begin{array}{l}20 \\
40 \\
23 \\
20 \\
50\end{array}$ & $\begin{array}{r}7.0 \\
50.0 \\
22.6 \\
16.5 \\
51.7\end{array}$ & $\begin{array}{l}60 \\
60 \\
60 \\
60 \\
60\end{array}$ & $\begin{array}{r}15.8 \\
16.1 \\
14.9 \\
4.7 \\
16.2\end{array}$ & $\begin{array}{l}140 \\
140 \\
141 \\
139 \\
t 40\end{array}$ & $\begin{array}{l}43.4 \\
58.0 \\
11.5 \\
22.5 \\
28.4\end{array}$ & $\begin{array}{r}12.3 \\
12.2 \\
5.2 \\
24.9 \\
0.2\end{array}$ & & $\begin{array}{l}6 \\
5 \\
6 \\
4 \\
5\end{array}$ & $\begin{array}{l}4 \\
2 \\
2 \\
3 \\
1\end{array}$ & $\begin{array}{l}144 \\
246 \\
177\end{array}$ & $\begin{array}{l}44 \\
44 \\
52 \\
49 \\
59\end{array}$ & $\begin{array}{l}0.33 \\
0.11 \\
0.09\end{array}$ & $\begin{array}{l}2.6 \\
4.3 \\
2.1 \\
6.2 \\
4.9\end{array}$ & $\begin{array}{l}3.6 \\
3.6 \\
6.9\end{array}$ & $\begin{array}{l}B \\
C \\
C\end{array}$ & $\begin{array}{r}93 \\
2 \notin 2 \\
73 \\
121 \\
300\end{array}$ & $\begin{array}{r}5 \\
5 \\
16 \\
7 \\
9\end{array}$ & $\begin{array}{l}0.8 \\
1.0 \\
0.8 \\
1.1 \\
1.1\end{array}$ & $\begin{array}{r}359 \\
15 \\
336 \\
213 \\
35\end{array}$ & $\begin{array}{l}36 \\
34 \\
22 \\
16 \\
30\end{array}$ & $\begin{array}{l}1.6 \\
1.5 \\
1.2 \\
\text { 1. } \\
\text { 2. } 0\end{array}$ & $\begin{array}{r}199 \\
185 \\
196 \\
\quad 8 \\
195\end{array}$ & $\begin{array}{l}56 \\
62 \\
72 \\
58\end{array}$ & $\begin{array}{l}3.6 \\
7.4 \\
4.0 \\
3.9 \\
7.9\end{array}$ \\
\hline $\begin{array}{l}7 \\
8 \\
9 \\
8 \\
8\end{array}$ & $\begin{array}{r}22 \\
1 \\
4 \\
4 \\
5\end{array}$ & $\begin{array}{r}55 \\
46 \\
0 \\
48 \\
26\end{array}$ & $\begin{array}{r}50.2 \\
6.5 \\
7.1 \\
40.6 \\
42.4\end{array}$ & $\begin{array}{l}60 \\
62 \\
60 \\
60 \\
60\end{array}$ & $\begin{array}{r}41.0 \\
7.9 \\
4.4 \\
13.1 \\
0.5\end{array}$ & $\begin{array}{l}141 \\
142 \\
140 \\
140 \\
140\end{array}$ & $\begin{array}{r}1.8 \\
23.8 \\
20.7 \\
59.3 \\
12.5\end{array}$ & $\begin{array}{r}17.9 \\
20.0 \\
20.3 \\
9.4 \\
14.5\end{array}$ & $\begin{array}{l}0.1 . \\
2.1 \\
1.1 \\
0.7 \\
0.5\end{array}$ & $\begin{array}{l}2 \\
6 \\
3 \\
4 \\
3\end{array}$ & $\begin{array}{l}3 \\
3 \\
1 \\
2 \\
1\end{array}$ & $\begin{array}{l}327 \\
370 \\
292 \\
148 \\
215\end{array}$ & $\begin{array}{r}91 \\
42 \\
70 \\
42 \\
59\end{array}$ & $\begin{array}{l}0.15 \\
1.19 \\
0.1 \\
0.02 \\
0 .\end{array}$ & $\begin{array}{r}17.6 \\
6.3 \\
16.5 \\
\text { B. } 5 \\
17.5\end{array}$ & $\begin{array}{r}9.6 \\
11.2 \\
10.0\end{array}$ & D & $\begin{array}{r}140 \\
14 \\
9 \\
277 \\
123\end{array}$ & $\begin{array}{r}1 \\
11 \\
30 \\
0 \\
19\end{array}$ & $\begin{array}{r}2.1 \\
9.4 \\
19.8 \\
1.2 \\
1.2\end{array}$ & $\begin{array}{r}234 \\
109 \\
259 \\
6 \\
229\end{array}$ & $\begin{array}{l}44 \\
25 \\
31 \\
37 \\
39\end{array}$ & $\begin{array}{l}\text { 2. } \\
\text { 2. } \\
4.9 \\
1.9 \\
2.9\end{array}$ & $\begin{array}{r}46 \\
262 \\
133 \\
197 \\
12\end{array}$ & $\begin{array}{l}46 \\
52 \\
44 \\
53 \\
46\end{array}$ & $\begin{array}{r}25.9 \\
4.7 \\
1.9 \\
13.9 \\
25.0\end{array}$ \\
\hline $\begin{array}{l}a \\
\theta \\
\theta \\
8 \\
\theta\end{array}$ & $\begin{array}{r}6 \\
7 \\
10 \\
13 \\
13\end{array}$ & $\begin{array}{l}29 \\
32 \\
47 \\
21 \\
45\end{array}$ & $\begin{array}{l}50.8 \\
15.9 \\
54.0 \\
45.5 \\
15.2\end{array}$ & $\begin{array}{l}60 \\
60 \\
34 \\
61 \\
60\end{array}$ & $\begin{array}{l}16.3 \\
11.9 \\
35.9 \\
47.3 \\
14.9\end{array}$ & $\begin{array}{l}141 \\
141 \\
139 \\
147 \\
140\end{array}$ & $\begin{array}{r}4.3 \\
1.1 \\
21.1 \\
28.5 \\
37.2\end{array}$ & $\begin{array}{r}0.3 \\
13.0 \\
17.3 \\
31.4 \\
12.2\end{array}$ & $\begin{array}{l}0 . \\
0 . \\
2 \\
3 \\
0 .\end{array}$ & $\begin{array}{r}5 \\
4 \\
16 \\
26 \\
4\end{array}$ & $\begin{array}{l}2 \\
1 \\
3 \\
2 \\
4\end{array}$ & & $\begin{array}{l}45 \\
44 \\
31 \\
69 \\
83\end{array}$ & $\begin{array}{l}0.22 \\
0.26 \\
0.79 \\
0.71 \\
0.10\end{array}$ & $\begin{array}{r}1.0 \\
11.0 \\
1.6 \\
1.6 \\
8.0\end{array}$ & $\begin{array}{r}3.9 \\
13.2 \\
1.1 \\
1.1 \\
9.7\end{array}$ & $\stackrel{A}{c}$ & $\begin{array}{r}268 \\
267 \\
180 \\
2 \\
109\end{array}$ & $\begin{array}{r}2 \\
6 \\
19 \\
9 \\
12\end{array}$ & $\begin{array}{l}0.8 \\
1.3 \\
1.6 \\
1.6 \\
1.0\end{array}$ & $\begin{array}{r}350 \\
23 \\
287 \\
95\end{array}$ & $\begin{array}{r}9 \\
41 \\
40 \\
16 \\
40\end{array}$ & $\begin{array}{l}0.8 \\
1.9 \\
0.9 \\
0.9 \\
1.5\end{array}$ & $\begin{array}{l}164 \\
190 \\
71 \\
244 \\
212\end{array}$ & $\begin{array}{l}82 \\
49 \\
44 \\
71 \\
49\end{array}$ & $\begin{array}{r}3.9 \\
17.6 \\
1.2 \\
1.1 \\
11.7\end{array}$ \\
\hline $\begin{array}{l}\mathrm{\theta} \\
\mathbf{6} \\
\mathrm{\theta} \\
\mathrm{\theta} \\
\theta\end{array}$ & $\begin{array}{l}14 \\
17 \\
17 \\
18 \\
18\end{array}$ & $\begin{array}{r}30 \\
18 \\
55 \\
2 \\
52\end{array}$ & $\begin{array}{l}16.7 \\
21.2 \\
50.0 \\
55.0 \\
28.0\end{array}$ & $\begin{array}{l}61 \\
60 \\
60 \\
60 \\
60\end{array}$ & $\begin{array}{r}26.9 \\
1.9 \\
12.3 \\
2.1 \\
0.9\end{array}$ & $\begin{array}{l}146 \\
140 \\
141 \\
141 \\
140\end{array}$ & $\begin{array}{l}42.2 \\
35.1 \\
15.7 \\
17.9 \\
14.0\end{array}$ & $\begin{array}{l}18.3 \\
12.3 \\
10.3 \\
15.1 \\
20.3\end{array}$ & $\begin{array}{l}1.8 \\
1.2 \\
1.4 \\
1.1\end{array}$ & $\begin{array}{r}16 \\
10 \\
9 \\
8 \\
4\end{array}$ & $\begin{array}{l}3 \\
2 \\
2 \\
3 \\
3\end{array}$ & & $\begin{array}{l}54 \\
51 \\
47 \\
55 \\
61\end{array}$ & $\begin{array}{l}0.59 \\
0.29 \\
0.12 \\
0.20 \\
0.32\end{array}$ & $\begin{array}{l}1.0 \\
2.0 \\
1.8 \\
1.4 \\
5.9\end{array}$ & $\begin{array}{l}1.9 \\
2.5 \\
2.1 \\
2.4 \\
3.3\end{array}$ & & $\begin{array}{l}106 \\
104 \\
310 \\
350 \\
109\end{array}$ & $\begin{array}{r}1 \\
6 \\
3 \\
12 \\
7\end{array}$ & $\begin{array}{l}0.6 \\
0.6 \\
1.0 \\
0.6 \\
0.9\end{array}$ & $\begin{array}{r}196 \\
10 \\
43 \\
04 \\
15\end{array}$ & $\begin{array}{r}3 \\
33 \\
40 \\
17 \\
23\end{array}$ & $\begin{array}{l}\text { 1. } 0 \\
1.6 \\
1.0 \\
1.1 \\
6.7\end{array}$ & $\begin{array}{l}750 \\
203 \\
216 \\
227 \\
224\end{array}$ & & $\begin{array}{l}\text { 2. } 8 \\
2.6 \\
2.6 \\
2.4\end{array}$ \\
\hline $\begin{array}{l}\text { 日 } \\
\text { 日 } \\
\text { 日 } \\
\text { 日 } \\
9\end{array}$ & $\begin{array}{r}19 \\
19 \\
20 \\
21 \\
1\end{array}$ & $\begin{array}{l}17 \\
29 \\
11 \\
32 \\
35\end{array}$ & $\begin{array}{l}57.6 \\
12.0 \\
33.7 \\
43.2 \\
19.3\end{array}$ & $\begin{array}{l}59 \\
60 \\
60 \\
50 \\
60\end{array}$ & $\begin{array}{l}54.4 \\
42.3 \\
15.5 \\
37.4 \\
44.1\end{array}$ & $\begin{array}{l}153 \\
147 \\
140 \\
137 \\
140\end{array}$ & $\begin{array}{r}24.3 \\
36.0 \\
56.6 \\
6.7 \\
42.2\end{array}$ & $\begin{array}{r}145.8 \\
27.7 \\
3.4 \\
11.7 \\
67.6\end{array}$ & & $\begin{array}{r}21 \\
18 \\
5 \\
7 \\
19\end{array}$ & $\begin{array}{r}1 \\
10 \\
3 \\
1 \\
1\end{array}$ & $\begin{array}{l}140 \\
117 \\
169 \\
356 \\
238\end{array}$ & $\begin{array}{r}90 \\
69 \\
109 \\
206 \\
129\end{array}$ & $\begin{array}{l}0.35 \\
0.39 \\
0.22 \\
1.02 \\
0.46\end{array}$ & $\begin{array}{r}2.8 \\
0.8 \\
1.0 \\
25.0 \\
6.5\end{array}$ & $\begin{array}{r}6.1 \\
1.9 \\
4.2 \\
48.6 \\
10.0\end{array}$ & $\begin{array}{l}\text { D } \\
\text { D }\end{array}$ & $\begin{array}{r}282 \\
329 \\
43 \\
260\end{array}$ & $\begin{array}{r}5 \\
4 \\
10 \\
1 \\
2\end{array}$ & $\begin{array}{r}2.7 \\
0.5 \\
1.1 \\
25.0 \\
2.0\end{array}$ & $\begin{array}{r}32 \\
192 \\
62 \\
312 \\
350\end{array}$ & $\begin{array}{r}13 \\
5 \\
18 \\
43 \\
14\end{array}$ & $\begin{array}{l}\text { 2. } \\
\text { o. } \\
\text { o. } \\
\text { s. } \\
\text { b. } 2\end{array}$ & $\begin{array}{l}234 \\
51 \\
211 \\
134 \\
162\end{array}$ & $\begin{array}{l}76 \\
94 \\
69 \\
47 \\
76\end{array}$ & $\begin{array}{r}6.3 \\
1.9 \\
4.5 \\
25.0 \\
10.2\end{array}$ \\
\hline 9 & 5 & 7 & 日. 1 & 60 & 49.0 & 139 & 31.7 & 2.0 .5 & $\begin{array}{r}1.9 \\
\text { EARC }\end{array}$ & 0 & 5 & 129 & 100 & .63 & 1. 4 & 6. & C & 100 & 3 & 1. & \$0 & 3 & .4 & 235 & 06 & a. 2 \\
\hline $\begin{array}{l}7 \\
9 \\
9 \\
9\end{array}$ & $\begin{array}{l}3 \\
5 \\
7 \\
9\end{array}$ & $\begin{array}{l}13 \\
39 \\
22 \\
14\end{array}$ & $\begin{array}{r}10.7 \\
5.9 \\
44.2 \\
41.0\end{array}$ & $\begin{array}{l}60 \\
60 \\
59 \\
60\end{array}$ & $\begin{array}{l}12.4 \\
14.4 \\
56.2 \\
12.9\end{array}$ & $\begin{array}{l}140 \\
142 \\
140 \\
141\end{array}$ & $\begin{array}{l}59.5 \\
34.4 \\
51.7 \\
29.4\end{array}$ & $\begin{array}{r}11.1 \\
3.7 \\
3.7 \\
5.4\end{array}$ & $\begin{array}{l}1.5 \\
1.4 \\
1.6 \\
1.6\end{array}$ & $\begin{array}{l}10 \\
10 \\
12 \\
14\end{array}$ & $\begin{array}{l}4 \\
3 \\
2 \\
5\end{array}$ & $\begin{array}{r}97 \\
136 \\
101\end{array}$ & $\begin{array}{l}39 \\
34 \\
41 \\
37\end{array}$ & $\begin{array}{l}\text { o. } 26 \\
0.27 \\
0.34\end{array}$ & $\begin{array}{l}1.5 \\
1.2 \\
1.7 \\
1.4\end{array}$ & $\begin{array}{l}\text { 2. } 5 \\
2.3 \\
1.9 \\
1.9\end{array}$ & $\hat{A}$ & $\begin{array}{l}345 \\
310 \\
109 \\
206\end{array}$ & $\begin{array}{r}19 \\
10 \\
8 \\
13\end{array}$ & $\begin{array}{l}0.7 \\
0.6 \\
0.6 \\
0.7\end{array}$ & $\begin{array}{l}8.2 \\
43 \\
14 \\
23\end{array}$ & & $\begin{array}{l}0.9 \\
1.0 \\
1.0 \\
1.0\end{array}$ & $\begin{array}{l}215 \\
192 \\
212 \\
173\end{array}$ & & $\begin{array}{l}\text { 2. } 8 \\
2.4 \\
2.0 \\
2.1\end{array}$ \\
\hline $\begin{array}{l}9 \\
9 \\
9 \\
9 \\
9\end{array}$ & $\begin{array}{l}7 \\
13 \\
14 \\
19 \\
15\end{array}$ & $\begin{array}{l}23 \\
12 \\
38 \\
19 \\
48\end{array}$ & $\begin{array}{r}32.5 \\
3.4 \\
10.9 \\
27.5 \\
30.9\end{array}$ & $\begin{array}{l}62 \\
60 \\
61 \\
60 \\
62\end{array}$ & $\begin{array}{l}\text { 5B. } 6 \\
11.6 \\
30.9 \\
14.9 \\
47.6\end{array}$ & $\begin{array}{l}149 \\
141 \\
150 \\
141 \\
149\end{array}$ & $\begin{array}{r}33.7 \\
10.8 \\
7.8 \\
18.9 \\
40.8\end{array}$ & $\begin{array}{r}40.9 \\
7.1 \\
41.5 \\
6.5 \\
49.9\end{array}$ & $\begin{array}{l}1.6 \\
2.1 \\
0.1 \\
2.4\end{array}$ & $\begin{array}{r}12 \\
15 \\
5 \\
10\end{array}$ & $\begin{array}{l}3 \\
4 \\
7 \\
2 \\
3\end{array}$ & $\begin{array}{r}113 \\
99 \\
153 \\
155\end{array}$ & $\begin{array}{r}196 \\
35 \\
55 \\
46 \\
132\end{array}$ & $\begin{array}{l}0.30 \\
0.30 \\
0.09 \\
0.41\end{array}$ & $\begin{array}{l}2.5 \\
1.6 \\
1.1 \\
2.4 \\
3.9\end{array}$ & $\begin{array}{r}2.8 \\
4.4 \\
25.0\end{array}$ & $\begin{array}{l}0 \\
0 \\
0\end{array}$ & $\begin{array}{r}153 \\
95 \\
257 \\
321 \\
268\end{array}$ & $\begin{array}{r}1 \\
10 \\
0 \\
16 \\
0\end{array}$ & $\begin{array}{l}2.3 \\
0.6 \\
0.8 \\
0.0 \\
2.2\end{array}$ & $\begin{array}{r}350 \\
267 \\
97 \\
339\end{array}$ & $\begin{array}{r}4 \\
41 \\
5 \\
19 \\
3\end{array}$ & $\begin{array}{l}\text { 1. } \\
\text { 1. } 6 \\
\text { 5. } 8\end{array}$ & $\begin{array}{l}257 \\
197 \\
347 \\
194 \\
178\end{array}$ & $\begin{array}{l}85 \\
65 \\
97\end{array}$ & $\begin{array}{r}25.0 \\
2.1 \\
2.0 \\
4.0 \\
25.0\end{array}$ \\
\hline $\begin{array}{r}9 \\
9 \\
9 \\
9 \\
10\end{array}$ & $\begin{array}{l}15 \\
16 \\
17 \\
21 \\
5\end{array}$ & $\begin{array}{l}50 \\
44 \\
54 \\
30 \\
94\end{array}$ & $\begin{array}{r}9.9 \\
29.9 \\
24.6 \\
41.5 \\
23.1\end{array}$ & $\begin{array}{l}60 \\
59 \\
60 \\
60 \\
50\end{array}$ & $\begin{array}{l}37.7 \\
13.4 \\
16.0 \\
47.3 \\
36.5\end{array}$ & $\begin{array}{l}147 \\
198 \\
141 \\
147 \\
155\end{array}$ & $\begin{array}{r}39.3 \\
13.0 \\
1.4 \\
40.7 \\
12.5\end{array}$ & $\begin{array}{r}1.9 \\
0.0 \\
1.4 \\
29.0 \\
144.6\end{array}$ & $\begin{array}{l}2.1 \\
1.3 \\
0.7 \\
2.0 \\
4.2\end{array}$ & $\begin{array}{r}20 \\
3 \\
5 \\
13 \\
7\end{array}$ & $\begin{array}{l}3 \\
2 \\
1 \\
4 \\
2\end{array}$ & $\begin{array}{r}62 \\
349 \\
166 \\
129 \\
234\end{array}$ & $\begin{array}{r}68 \\
114 \\
61 \\
74 \\
106\end{array}$ & $\begin{array}{l}0.26 \\
0.21 \\
0.47 \\
0.29 \\
0.24\end{array}$ & $\begin{array}{r}0.9 \\
24.7 \\
2.1 \\
2.4 \\
6.8\end{array}$ & $\begin{array}{r}1.2 \\
7.8 \\
5.3 \\
2.3 \\
10.5\end{array}$ & D & $\begin{array}{l}156 \\
20 \% \\
357 \\
260 \\
325\end{array}$ & $\begin{array}{r}12 \\
9 \\
13 \\
0 \\
0\end{array}$ & $\begin{array}{r}0.9 \\
25.0 \\
0.9 \\
0.7 \\
2.9\end{array}$ & $\begin{array}{r}255 \\
117 \\
90 \\
170 \\
35\end{array}$ & $\begin{array}{l}24 \\
12 \\
14 \\
16 \\
21\end{array}$ & $\begin{array}{l}\text { 9. } 9 \\
1.3 \\
\text { 2. } 2 \\
5.9\end{array}$ & $\begin{array}{r}45 \\
335 \\
226 \\
350 \\
235\end{array}$ & $\begin{array}{l}75 \\
71 \\
74 \\
69\end{array}$ & $\begin{array}{r}1.3 \\
6.7 \\
5.6 \\
2.4 \\
11.0\end{array}$ \\
\hline
\end{tabular}




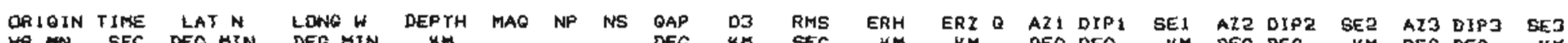

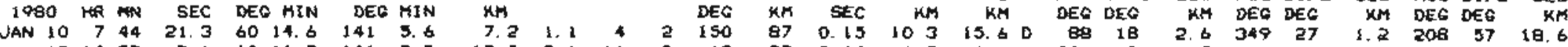

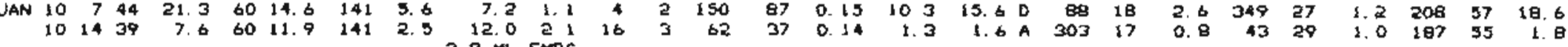

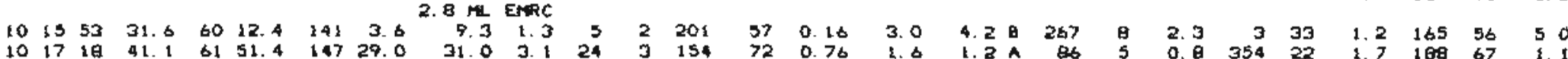

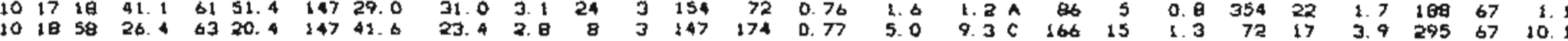

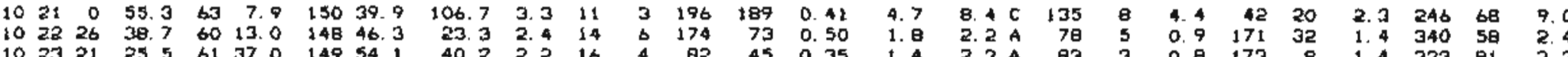

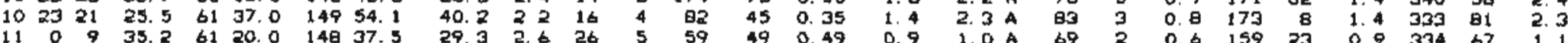

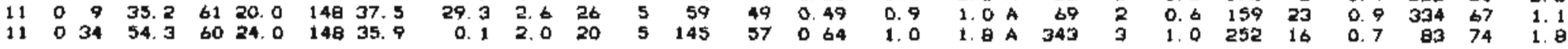

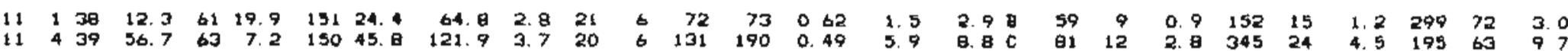

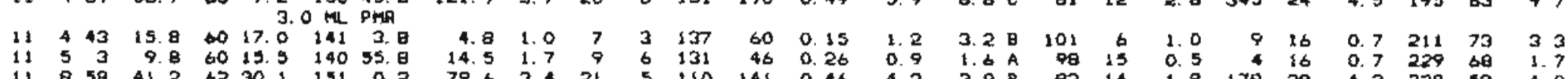

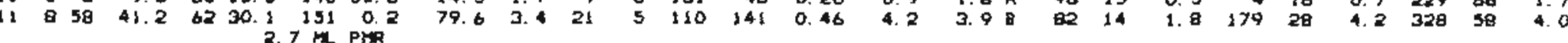

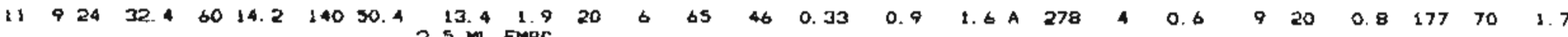

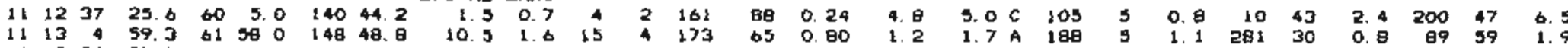

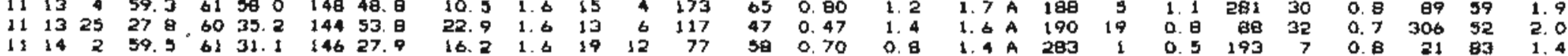

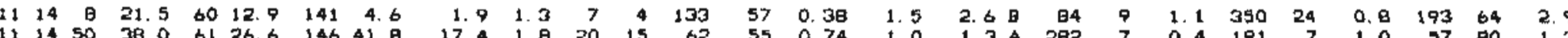

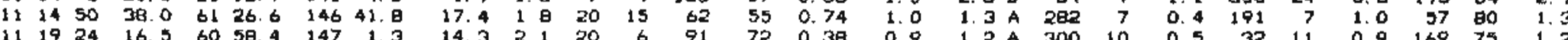

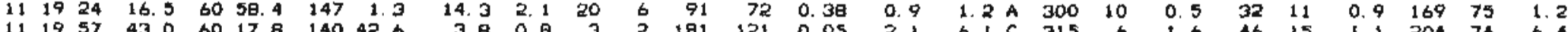

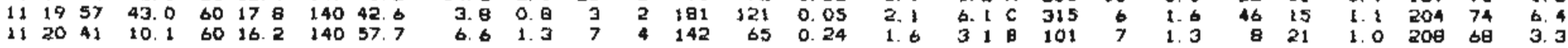

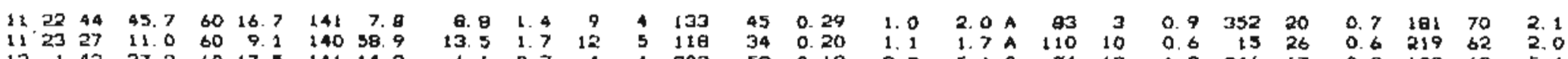

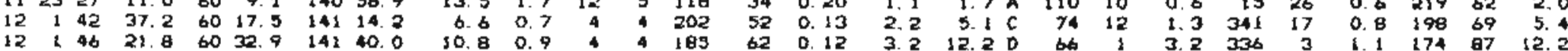

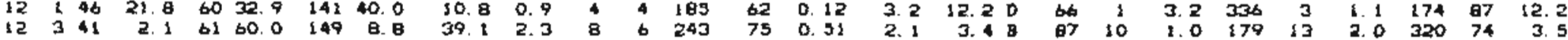

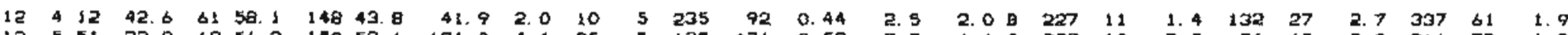

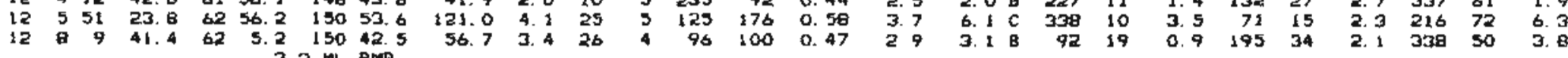

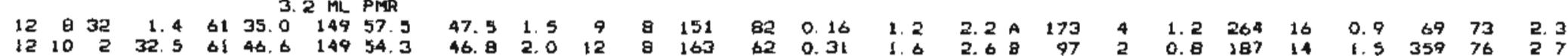

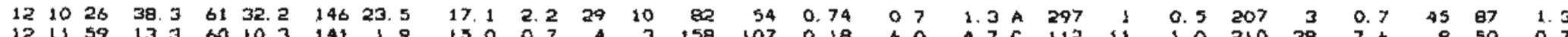

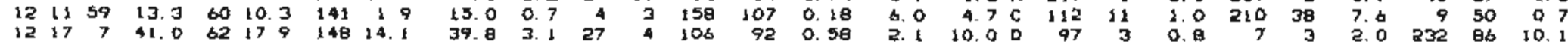

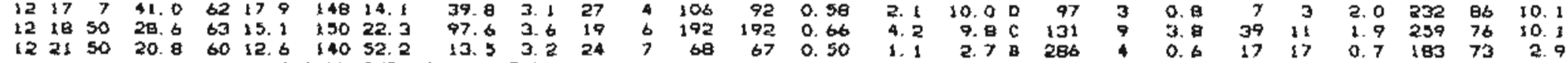

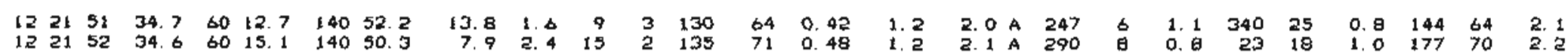

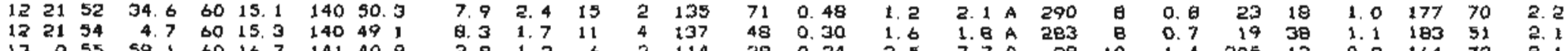

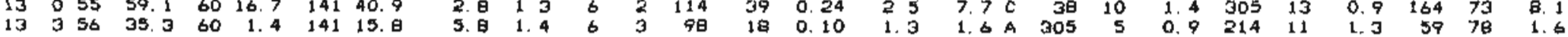




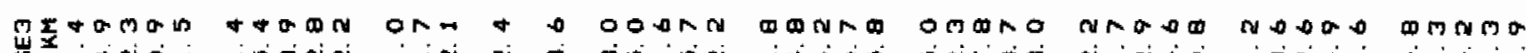

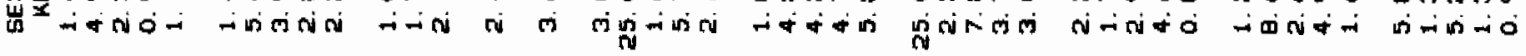

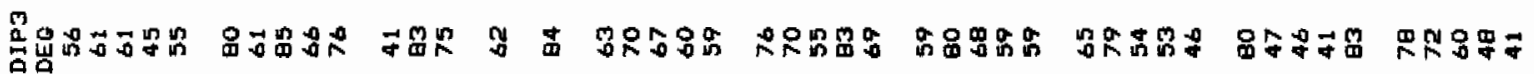

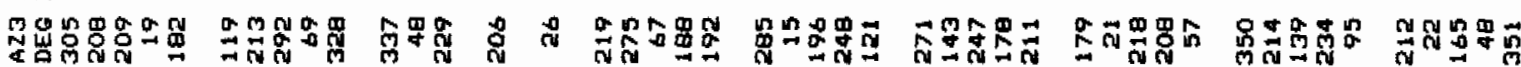

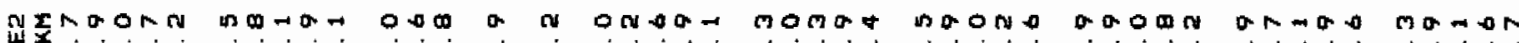
㟧

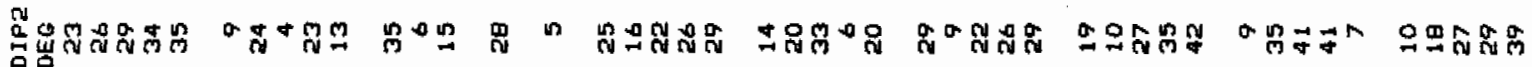

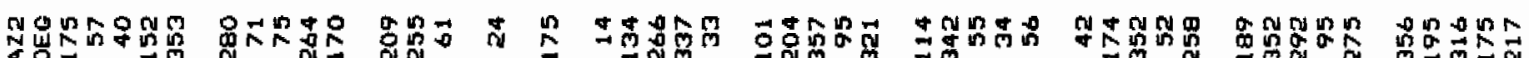

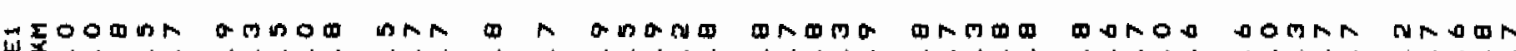

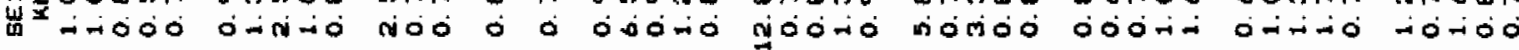

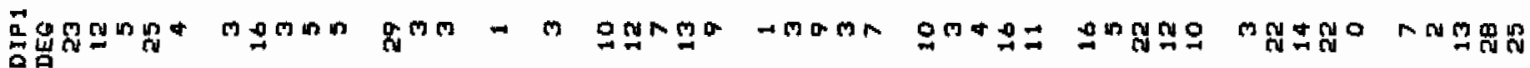

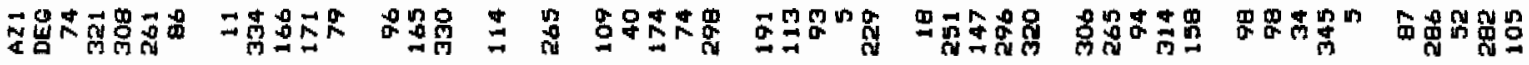

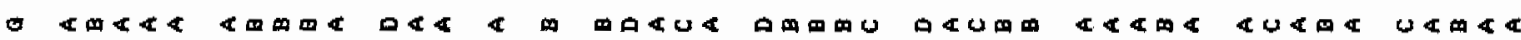

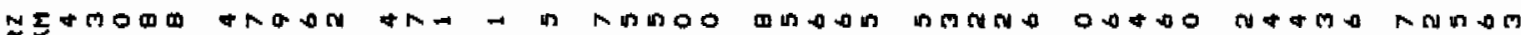
यุ

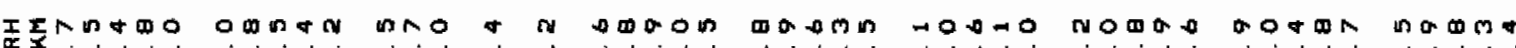
III -

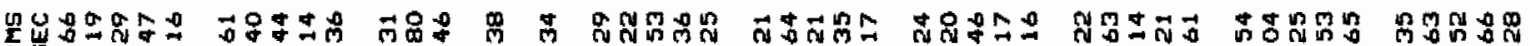

íóó ióó

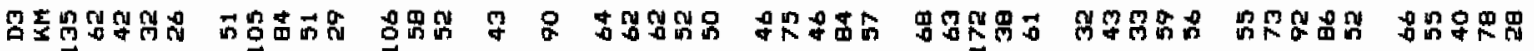

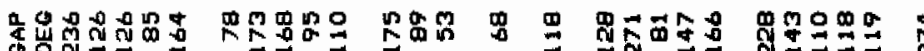

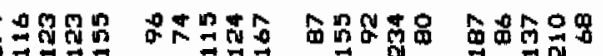

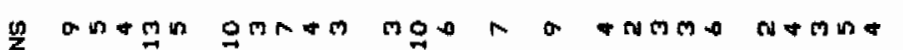

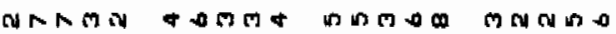

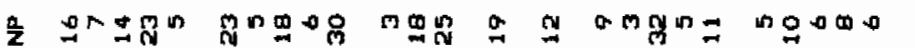

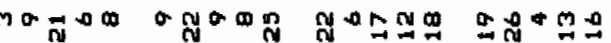

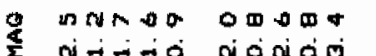

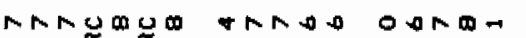

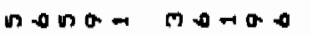

On 0 कात

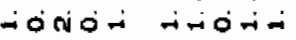

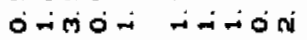

0 or on

0.0 in 0 o 0

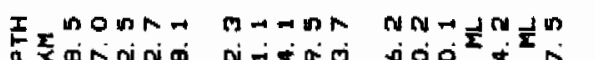

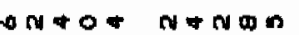

notor $n-\infty \circ$

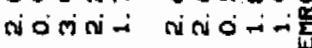

岩

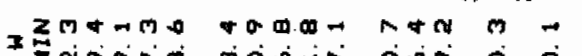

की

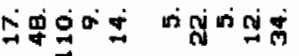

omon NaOd

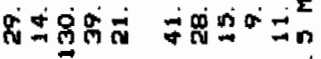

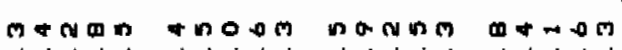

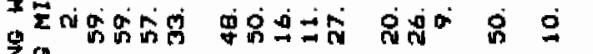

OMnO Un DNR

界-q日日

$\rightarrow$ in

मूंकुण तु

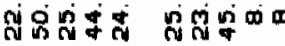

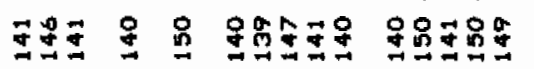

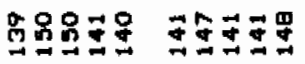

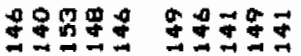

Mnd 0 क

OMN NO ONN+0

0 moन $400 \mathrm{O}$

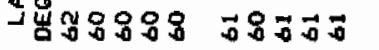

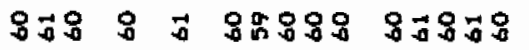

- món

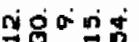

कि

ixim

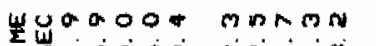

-0+ a n tanno noma

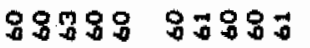

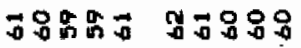

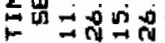

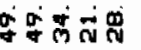

该寉

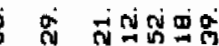

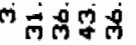

N⿴囗十力 人on

DOM-O MMNNO

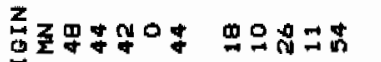

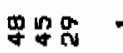

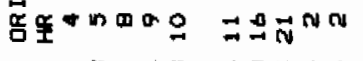

$\operatorname{tin} 0$

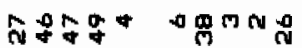

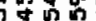

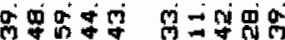

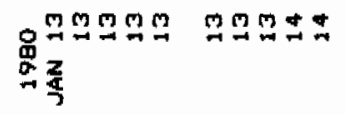

a gavar

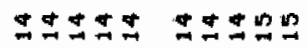

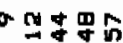

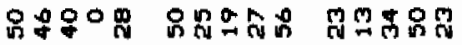

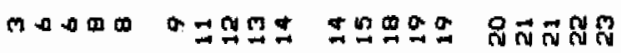

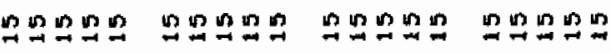


SONTHERN ALASKA EARTHOUAKES, JAN - MAR 1980

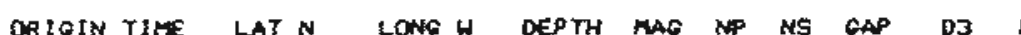
1980 HR MN SEC DEE MIN DEG MIN KM TH JAN 16 042 G. 0 Q $0021.0 \quad 14120.3$ 10.

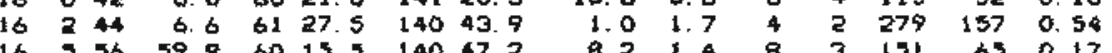

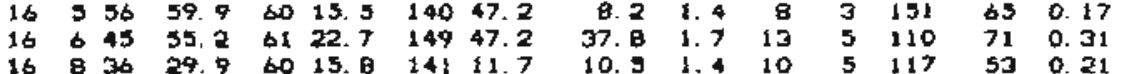

117

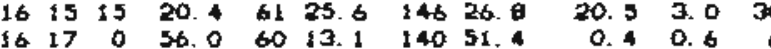

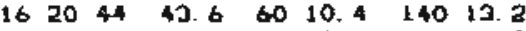
17339205 60 10.5142 10. 3

$17519 \quad 3.2$ 60 39.7 147 39.9

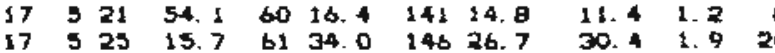
17 6 $16 \quad 34.2 \quad 5913.6 \quad 136$ i3. 1

$17749 \quad 56.4$ 60 $1.5 \quad 14116.5 \quad 4.00 .9$

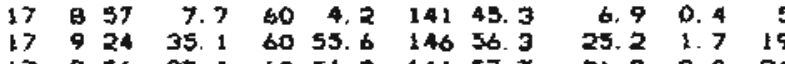
17 . 59 37. $6054.714657,7$

$171746 \quad 40.9 \quad 5726.9 \quad 15053.2$ 21.93 .034

$\begin{array}{ll}1 & 277 \\ 0 & 125\end{array}$

53 0. 21

ERH ERI O ALI DIP1 SEI AIZ DIPZ SE2 AL3 DIPJ SE3

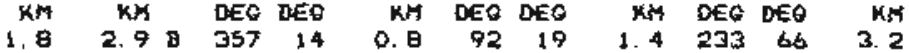

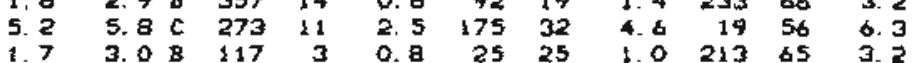

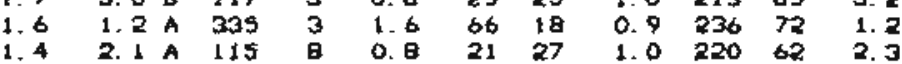

17 18 $\begin{aligned} & 3.6 \\ & 12\end{aligned}$

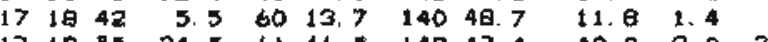

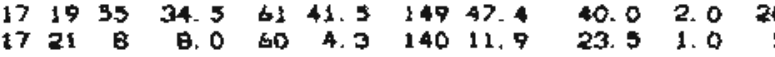

$\begin{array}{llllllll}17 & 21 & 36 & 56.7 & 60 & 2.1 & 140 & 36.0\end{array}$

$18 \quad 141 \quad 59.7$ 60 5.7 140 35.5

$\begin{array}{lllllllll}10 & 2 & 49 & 34 & 5 & 59 & 57.0 & 141 & 37.6 \\ 19 & 3 & 35 & 37 & 0 & 61 & 17.4 & 149 & 56.3\end{array}$

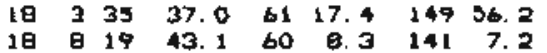

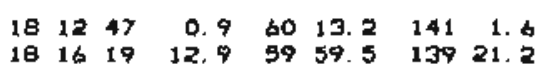

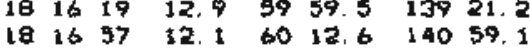

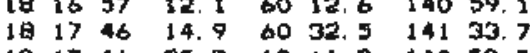

$\begin{array}{rr}\text { A. } 3 & \text { 1. } 3 \\ \text { 13. } 7 & \text { 0. }\end{array}$

0. $1.430 \quad 2106$

$\begin{array}{rrrrr}36.7 & 2.3 & 18 & 7 & 52 \\ 0.8 & 0.9 & 4 & 1 & 183\end{array}$

b7 $011310.6 \quad 23.10$ 26 10

o. $9 \quad 2127$

$191746 \quad 35.98011 .9030$. 5

4.40 .9

$\begin{array}{ll}15.0 & 0.5 \\ 15.0 & 0.6\end{array}$

$\begin{array}{rl}19.0 & 1.3 \\ 4.7 & 1.3\end{array}$

$\begin{array}{llllllll}19 & 1 & 16 & 46.6 & 60 & 39.6 & 141 & 23.5\end{array}$

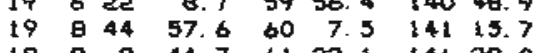

$1999 \quad 44.7$ 61 33.1 146 290

10. 0.9 7. 5 . 1.5 28. 0 2. 319

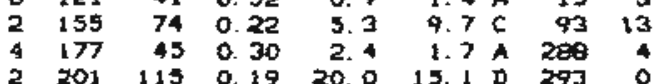

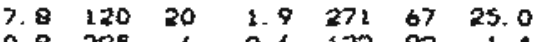
$\begin{array}{lllllll}0.9 & 285 & 6 & 0.6 & 132 & 93 & 1.4 \\ 1.2 & 357 & 24 & 1.4 & 209 & 62 & 10.5\end{array}$

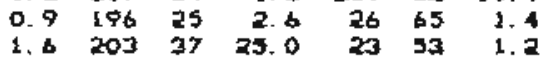

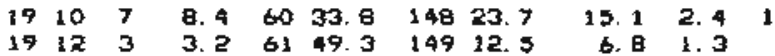
$9 \quad 1240 \quad 12.6 \quad 59 \quad 10.6 \quad 130 \quad 16.9$ $\begin{array}{llllllll}20 & 3 & 51 & 12.9 & 60 & 16.4 & 140 & 7.2 \\ 20 & 7 & 42 & 11.1 & 59 & 56.9 & 139 & 47.5\end{array}$

o. 1.2 $\begin{array}{rr}\text { a. } 1 & 0.9 \\ 10.0 & 1.1\end{array}$ $\begin{array}{lllllll}0.8 & 170 & 42 & 5.5 & 955 & 40 & 1.1 \\ 0.9 & 96 & 21 & 1.2 & 220 & 60 & 2.9 \\ 0.7 & 184 & 18 & 6.9 & 20 & 71 & 1.1\end{array}$ $\begin{array}{rrrrrrr}0.7 & 194 & 16 & 0.9 & 20 & 75 & 2.1 \\ 3.2 & 49 & 42 & 10.0 & 214 & 47 & 2.2\end{array}$

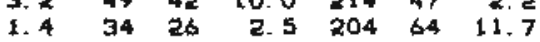
$\begin{array}{rrrrrrr}0.9 & 12 & 30 & 2.9 & 206 & 51 & 1.4 \\ 2.0 & 255 & 6 & 0.9 & 117 & 79 & 1.6\end{array}$

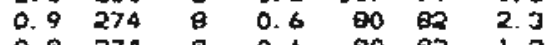
$\begin{array}{lrrrrrr}0.9 & 274 & 9 & 0.6 & 80 & 42 & 1.2 \\ 0.4 & 351 & 25 & 0.8 & 207 & 72 & 2.1\end{array}$ $\begin{array}{lllllll}25.0 & 45 & 30 & 25.0 & 183 & 52 & 25.0\end{array}$

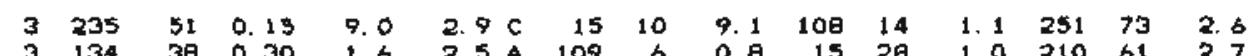

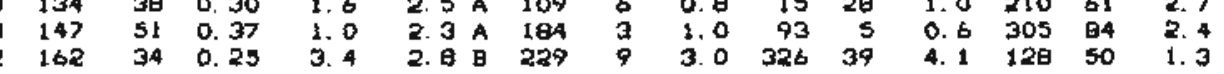
$\begin{array}{rrrrrrrrrrrrrr}39 & 0.44 & 1.9 & 2.1 & \text { A } & 106 & 3 & 0.7 & 197 & 22 & 1.7 & 9 & 66 & 2.2\end{array}$

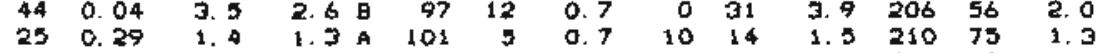

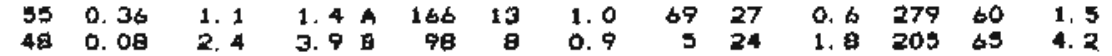
$\begin{array}{rrrrrrrrrrrrrrr}146 & 45 & 0.09 & 1.7 & 3.2 & 1 & 313 & 9 & 1.0 & 46 & 24 & 0.1 & 206 & 65 & 3.5 \\ 261 & 66 & 0.07 & 15.0 & 20.1 & 0 & 13 & 14 & 5.1 & 112 & 33 & 1.1 & 263 & 53 & 25.0\end{array}$

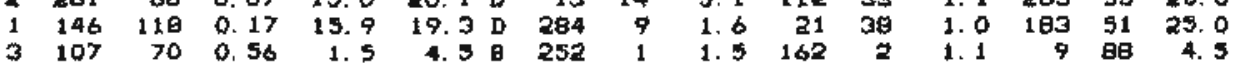

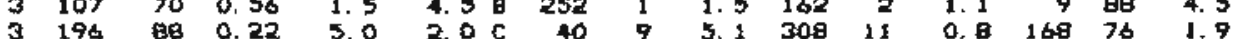

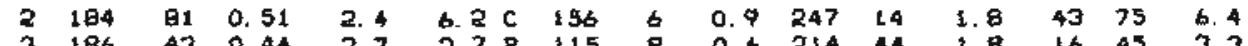
$\begin{array}{llllllllllllll}351 & 56 & 0.06 & 3.7 & 1.98 & 206 & 22 & 3.9 & 105 & 25 & 0.6 & 332 & 56 & 1.3\end{array}$

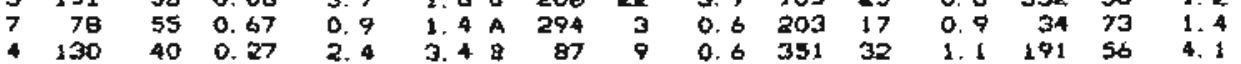

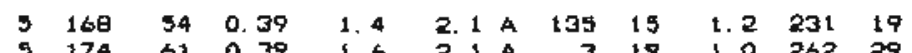

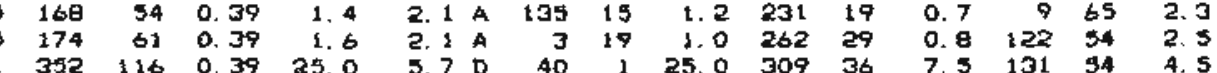

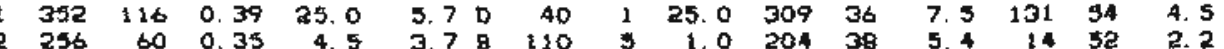

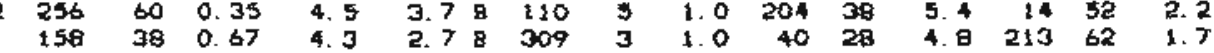


SOUTHERN ALASKA EAATHOUAKEE, JAN - MAF 1900

DRTOIN TIME LAT N LONE W DEPTH MAG NP NG GAP DJ RME 1960 HR MN BEC DEG MIN DEQ MIN KM MAO NP NB DEP

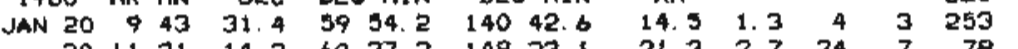
$\begin{array}{llllllll}20 & 11 & 41 & 49.6 & 60 & 15.3 & 140 & 40.4\end{array}$

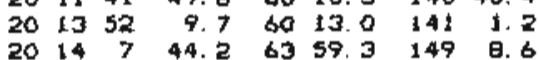

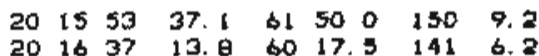
20 16 37 13. 1 6 60 17.5 141 6. $\begin{array}{llllllll}21 & 1 & 95 & 33.1 & 60 & 14.6 & 141 & 16.5 \\ 21 & 1 & 55 & 24.1 & 60 & 55.8 & 147 & 27.1\end{array}$ $21 \quad 320 \quad 46.7$ 60 54.9 147 27.7 $24 \quad 4 \quad 23 \quad 0.3 \quad$ b0 $16.8 \quad 140 \quad 493$

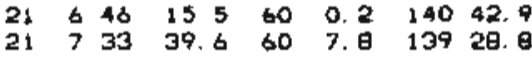
249 16 $56.6 \quad 6014.0 \quad 141 \quad 1.6$

2) $1726 \quad 10.7 \quad 60 \quad 16.7 \quad 141 \quad 3.3$ 211634 40. 1 60 13.0 141 1.2

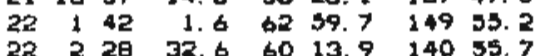

350.56 $\begin{array}{llll}4 & 152 & 46 & 0.21 \\ 44 & 0.10\end{array}$ 30. 0 2. 6 6

13. 317

4. $0.65 \%$

10. 0.5 \& 340

$\begin{array}{lllll}20.0 & 2.2 & 20 & 6 & 129 \\ 29.0 & 1.6 & 15 & 7 & 132\end{array}$

12.983143

13. 3 0.9

1.0

B. 5 0.

7. 0.7

Q. 7 3. 6

$22596 \quad 12.6 \quad 6056.4 \quad 14926.3$ $22 \quad \begin{array}{lllllll}56 & 26.6 & 60 & 9.1 & 140 & 53.1 \\ 25 & 536 & 7.3 & 60 & 5.4 & 140 & 51.0\end{array}$ $\begin{array}{rrrrrrrr}22 & 5 & 36 & 7.3 & 60 & 15.4 & 140 & 51.0 \\ 22 & 7 & 45 & 41.4 & 60 & 7.0 & 141 & 5.7\end{array}$
$22746 \quad 26.7$ bo $7.6 \quad 141 \quad 5.7$ 2. 日.9L EMAC

$22 \quad$ 日 $17 \quad 57.9$ 60 7.9 141 5 .

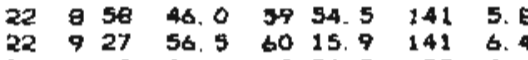
22110046.1 60 20.5 152 14.6

22 16 $7 \quad 0.4 \quad 6013.5 \quad 14058.4$ $\begin{array}{llllllll}22 & 16 & 41 & 10.7 & 59 & 56.1 & 145 & 51.2 \\ 22 & 18 & 54 & 59.3 & 60 & 7.7 & 141 & 4.8\end{array}$ $\begin{array}{llllllll}22 & 18 & 56 & 17.0 & 61 & 25.3 & 149 & 56.7 \\ 22 & 19 & 22 & 55.9 & 59 & 14.3 & 139 & 55.1\end{array}$

$\begin{array}{llllllll}22 & 20 & 44 & 0.9 & 61 & 1.4 & 149 & 56.0\end{array}$ $22 \quad 23 \quad 1 \quad 51.8$ 61 $30.9 \quad 150$ 15. $\begin{array}{llllllll}23 & 0 & 33 & 51.3 & 59 & 54.9 & 139 & 45.2 \\ 23 & 1 & 49 & 28.5 & 60 & 14.8 & 141 & 4.1\end{array}$

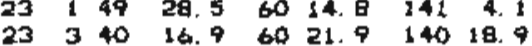
$2347 \quad 46.8 \quad 6014.5 \quad 14044$. 23457 51.5 $60 \quad 7.3 \quad 14026$. 23 i2 19 32. 0 60 1.3141 9. $\begin{array}{llllllll}23 & 12 & 19 & 32.0 & 60 & 1.3 & 141 & 9.1 \\ 23 & 12 & 19 & 41.0 & 60 & 13.7 & 141 & 0 .\end{array}$

\begin{abstract}
. 1 ML EMRC
\end{abstract}
$\begin{array}{lllll}7.5 & 1.6 & 9 & 4 & 103\end{array}$

$\begin{array}{lllll}0.7 & 1.3 & 6 & 3 & 209\end{array}$

86. 1 3. 4 24 3 日3

$26.7 \quad 1.2 \quad 5 \quad 4 \quad 152$

3. 30.92525149

日. 1 1. 5 5 3129

$\begin{array}{lllll}32 . & 2.4 & 14 & 12 & 61 \\ 3.5 & 3 & 291\end{array}$

54. 5 1.6 6 क 120

43. 2 2.0 $19 \quad 9 \quad 105$

$\begin{array}{lllll}15.5 & 0.9 & 4 & 1 & 264 \\ 15.0 & 0.9 & 3 & 3 & 277\end{array}$

$\begin{array}{lllll}19.0 & 0.9 & 3 & 3 & 227 \\ 10.9 & 1.0 & 6 & 1 & 197\end{array}$

$\begin{array}{rrrrr}9.9 & 1.0 & 5 & 3 & 166 \\ 12.9 & 1.3 & 4 & 1 & 304\end{array}$

$\begin{array}{llll}12.9 & 1.9 & 4 & 304 \\ 14.9 & 0.9 & 4 & 156\end{array}$

$\begin{array}{lllll}15.1 & 0.8 & 3 & 2 & 227\end{array}$
ERT O AZ1 DIPI SE1 AZE DIPE EEZ AZ3 DIP3 BE3 K. B C OEG DEQ KH DEG DEE KH DEG DEG MH $\begin{array}{llllllllll}1.4 A & 261 & 3 & 0.7 & 171 & 5 & 09 & 22 & 84 & 1.5\end{array}$

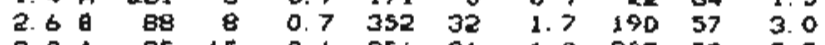
$\begin{array}{rrrrrrr}0.7 & 356 & 31 & 1.9 & 207 & 55 & 2.3 \\ 1.5 & 94 & 27 & 10.1 & 327 & 52 & 25.0\end{array}$ $\begin{array}{rrrrrrrrrrrrrr}73 & 0.65 & 2.0 & 1.7 & \text { A } & 291 & 12 & 0.7 & 17 & 25 & 2.0 & 168 & 42 & 1.6 \\ 52 & 0.19 & 1.6 & 4.0 & 8 & 305 & 5 & 0.9 & 37 & 18 & 0.9 & 200 & 71 & 4.2\end{array}$ $\begin{array}{llllllllllllll}0 . & 12 & 7.5 & 11.0 & \mathrm{D} & 100 & 11 & 0.9 & 3 & 32 & 0.9 & 207 & 56 & 13.3\end{array}$

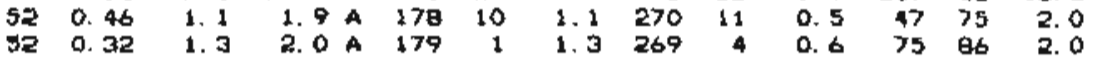
$42 \begin{array}{llllll}4.15 & 1.3 & 2.8 & B & 321 & 0\end{array}$ $\begin{array}{lllllll}32 & 0.18 & 11.0 & 8.40 & 121 & 13 \\ 45 & 0.25 & 2.1 & 2.8 & 0 & 320 & 23\end{array}$ 2. 8 : 32923 $\begin{array}{lllllll}\text { 1. } & 54 & 21 & 0.9 & 209 & 67 & 3.1 \\ 0.5 & 23 & 26 & 1.2 & 193 & 64 & 1.5\end{array}$

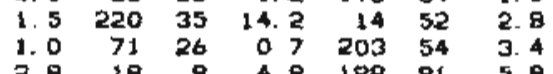
$\begin{array}{llllllllllllll}50 & 0.19 & 1.9 & \text { J. } 3 & 8 & 325 & 13 & 1.0 & 60 & 23 & 0.8 & 200 & 63 & 3 .\end{array}$

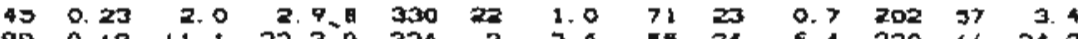

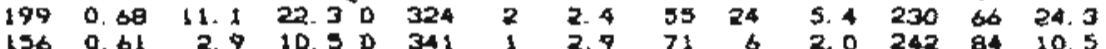

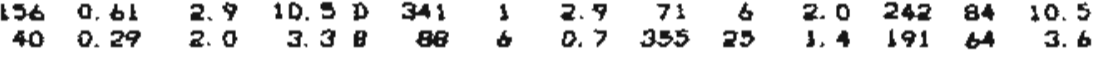

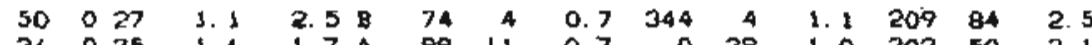

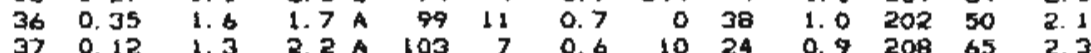

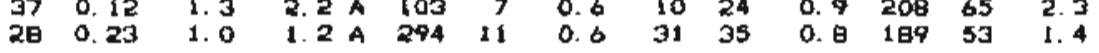

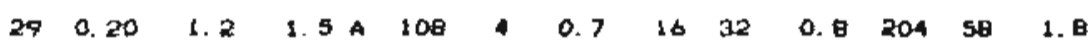

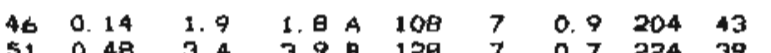

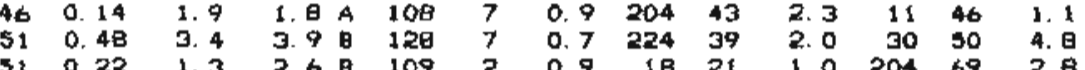

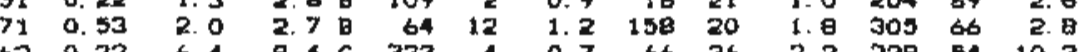

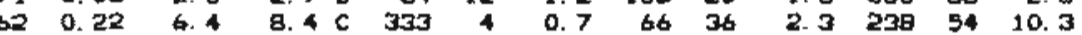

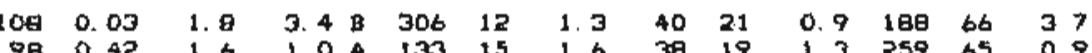

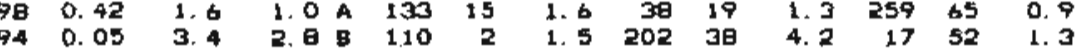

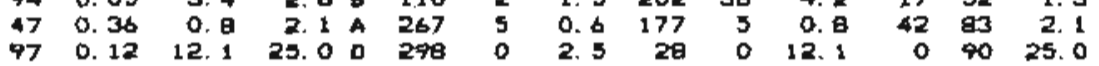
$\begin{array}{llllllllllllll}67 & 0.12 & 1.9 & 4.5 & 8 & 250 & 1 & 1.5 & 340 & 2 & 1.9 & 133 & 88 & 4.5 \\ 60 & 0.42 & 1.1 & 2.4 & 4 & 263 & 0 & 0.6 & 173 & 7 & 1.0 & 353 & 03 & 2.4\end{array}$ $\begin{array}{llllllllllllll}52 & 0.03 & 6.4 & 2.4 & \text { A } & 263 & 0 & 0.6 & 173 & 7 & 1.0 & 353 & 03 & 2.4 \\ 4 & 0.20 & 1.4 & 9.4 & 320 & 1 & 1.5 & 54 & 29 & 4.0 & 219 & 60 & 11.0\end{array}$

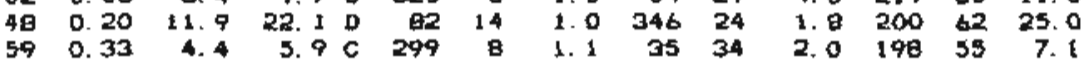
$47 \begin{array}{lllllllllll}0.34 & 2.2 & 3.5 & 9 & 81 & 9 & 0.9 & 346 & 27 & 1.3 & 1889\end{array}$

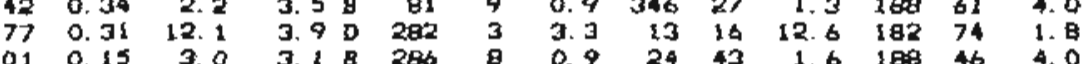

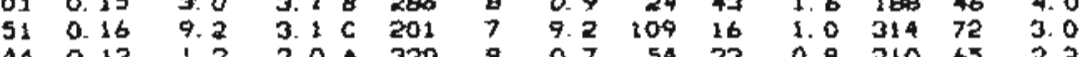




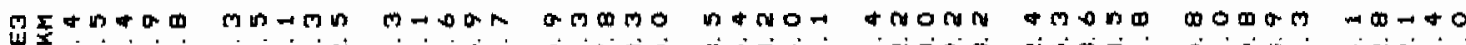
岩

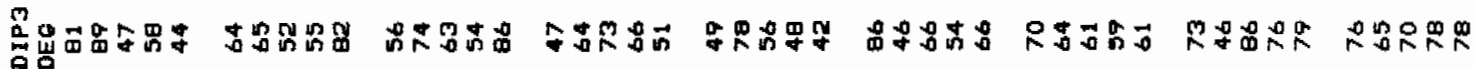

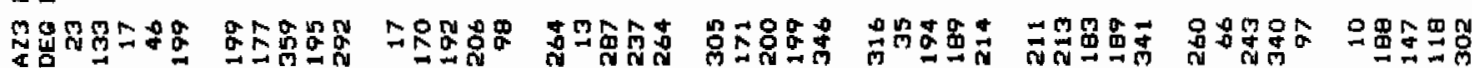

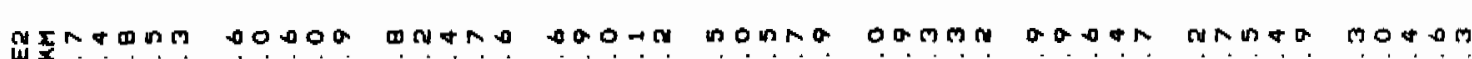
岁

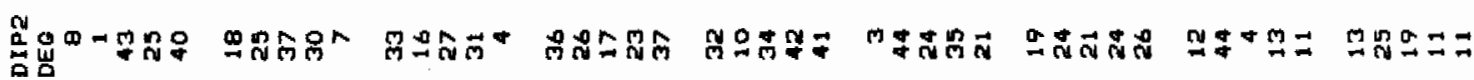

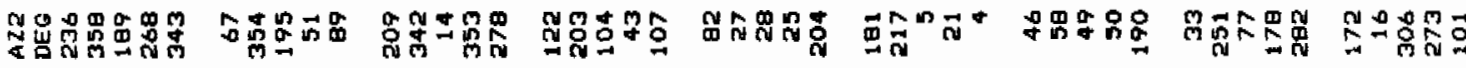

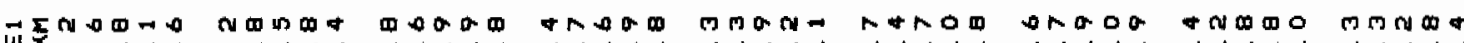

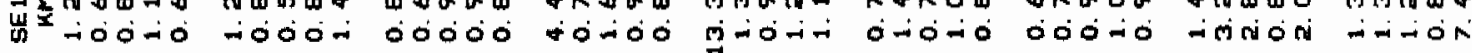

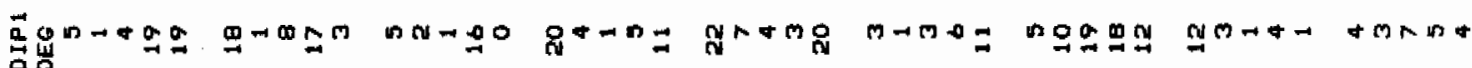

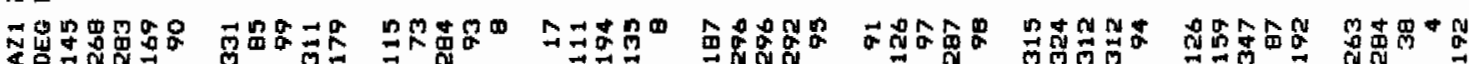

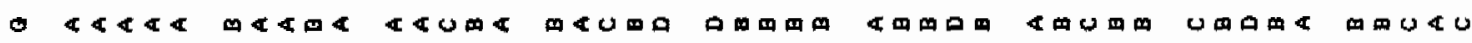
N5tnNM-

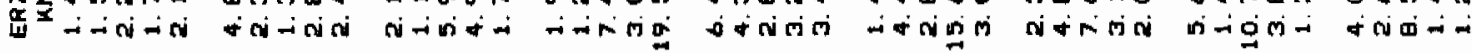

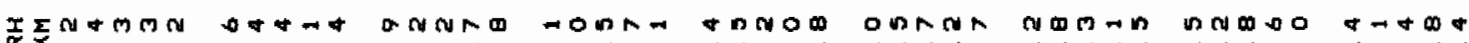
造

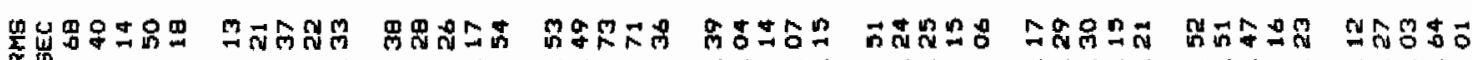

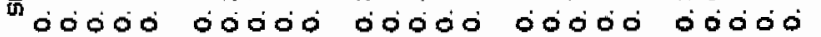

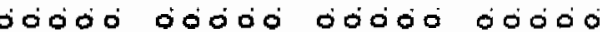

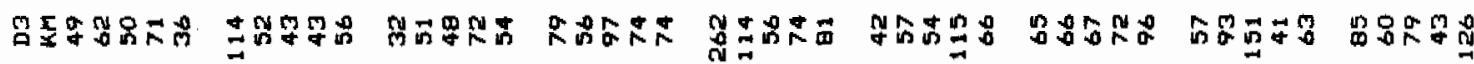

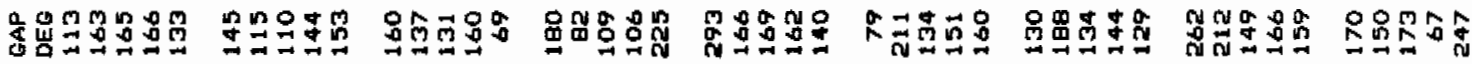

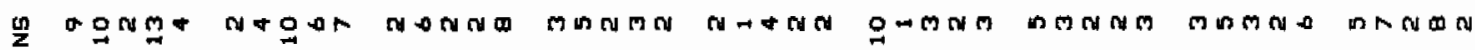

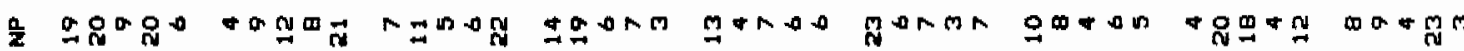

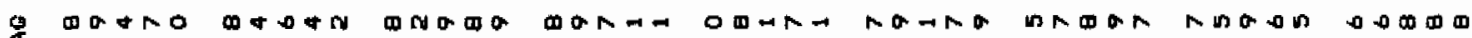
I

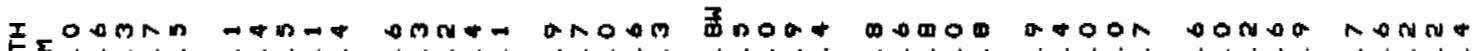

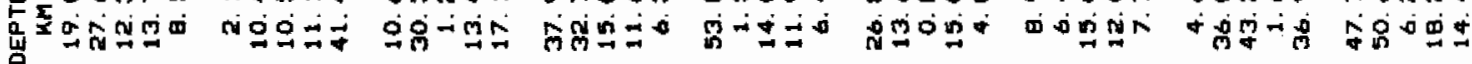

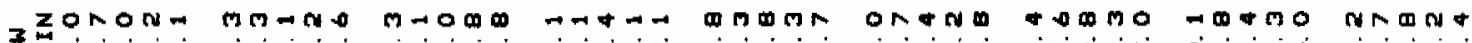

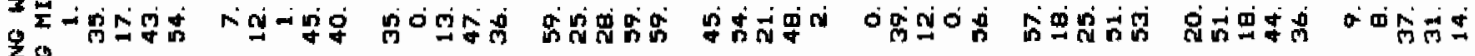

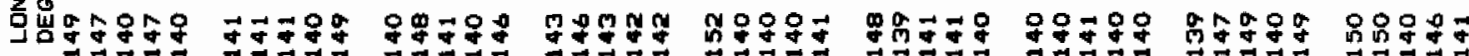

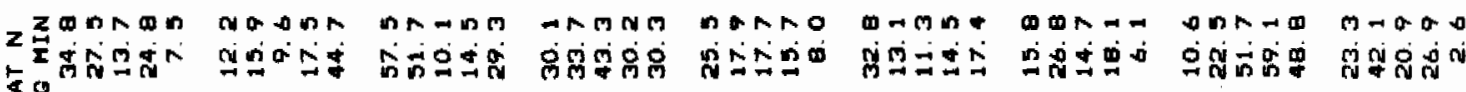

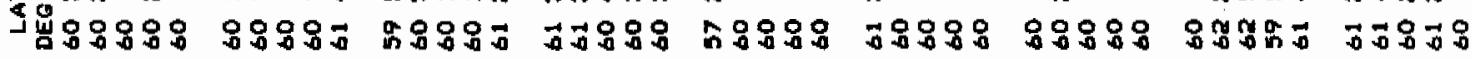

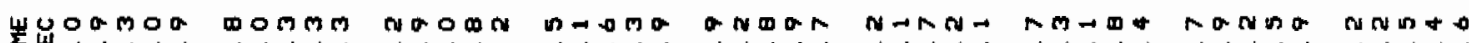

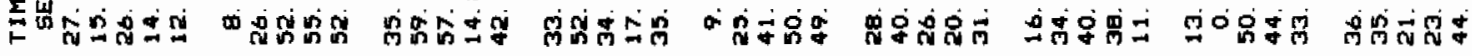

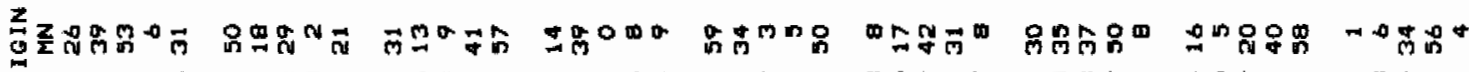

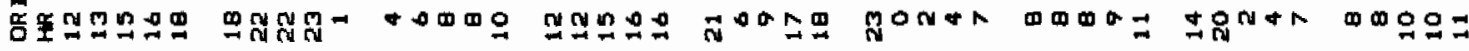

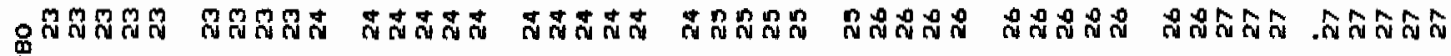
遂 
1960 URIOIN TIRE LAT N

19BO HAR MN SEC DEG MIN

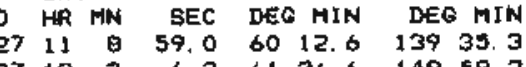

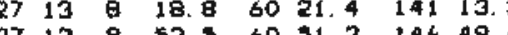
271414.960242145 .

$271451 \quad 1.1$ ol $52.7 \quad 14939.4$ $271934 \quad 97.3$ 60 15.14049 .1 $\begin{array}{llllllll}27 & 19 & 5 & 36.5 & 59 & 22.6 & 438 & 44.7\end{array}$ $\begin{array}{rrrrrrrr}27 & 19 & 52 & 46.4 & 60 & 16.3 & 140 & 59.9 \\ 27 & 20 & 51 & 2.1 & 60 & 17.5 & 140 & 45.9\end{array}$

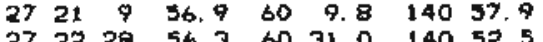
2722 26 $54.360 \quad 31.0 \quad 140 \quad 52.5$

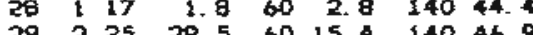

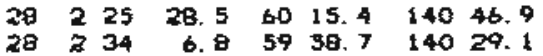

$2842917.4 \quad 6019.1 \quad 14043.5$ 287 a $58.6 \quad 6014.8 \quad 140 \quad 36.6$ 28 11 30 13.0 60 17.9 140 43.7

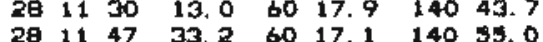

$281355 \quad 40.9$ t0 $140 \quad 14057.0$ 28 14 59 28.1 62 3. B 147 47. $28 \quad 1012$ 36.2 6011.7 141 9.9

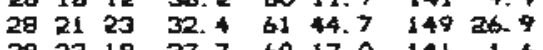

DEPTH

$\begin{array}{ll}\text { 13. } 1 & 0.8\end{array}$

$\begin{array}{rrrrr}13.1 & 0.8 & 3 & 3 & 330 \\ 47.7 & 1.9 & 17 & 6 & 78 \\ 13.9 & 1.4 & 9 & 2 & 122\end{array}$ $11.6 \quad 19 \quad 9 \quad 102$

5. $81.913 \div 16$ 11.5 $1.1 \quad 7 \quad 2 \quad 336$ 23. $4 \begin{array}{llll}1.3 & 5 & 2 & 337\end{array}$ $\begin{array}{lllll}\text { 12. } 1.5 & 1.3 & 7 & 2 & 128 \\ 11.9 & 1.5 & 7 & 3 & 144\end{array}$

9. 7 1. 0

$+2138$

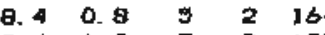
$\begin{array}{rrrrr}13.4 & 1.2 & 7 & 3 & 139 \\ 2.6 & 0.9 & 6 & 3 & 143\end{array}$

$0.9 \quad 0.9$ 13. 31.4 10.41 .17272129 $\begin{array}{ll}1 & 272 \\ 2 & 129 \\ 2 & 140\end{array}$ 3. 1 0.7

7.9 1.2 $6.72 .0 \quad 16 \quad 5 \quad 132$ 6.0 1. 272 91.094160 $282319 \quad 37.7 \quad 6017.0 \quad 141,11.6$ $\begin{array}{rrrrrrrr}29 & 0 & 13 & 50.5 & 60 & 14.9 & 140 & 56.5 \\ 27 & 11 & 14 & 51.7 & 62 & 37.1 & 149 & 3.2\end{array}$

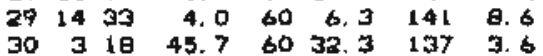
$\begin{array}{rrr}9.3 & 0.7 & 5 \\ 39.0 & 2.6 & 27\end{array}$ 4. 0.9

30435 35. 2 60 27.5 1513.3 2. 34.3 M.1 19

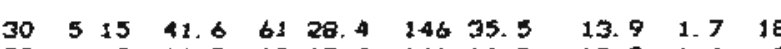

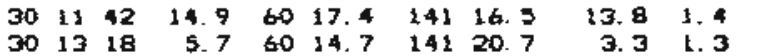

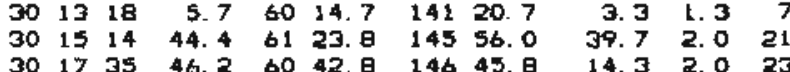

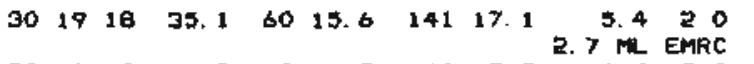

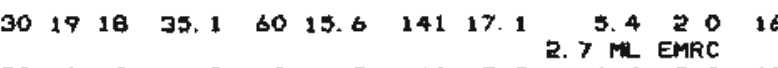

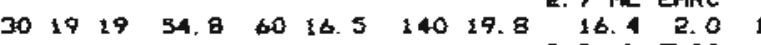
$\begin{array}{lllllllll}30 & 1956 & 20.1 & 61 & 1.9 & 147 & 37.6 & 20.0 & 1.7\end{array}$ $302274 \quad 8161537 \quad 14970.541 .51 .9$

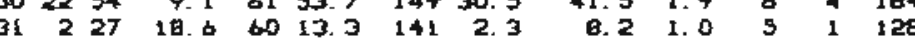

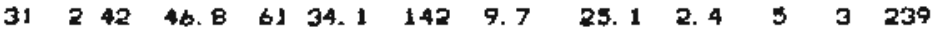
2. 5 HATC $\begin{array}{llllllll}31 & 5 & 52 & 16.5 & 61 & 37.1 & 142 & 2 .\end{array}$

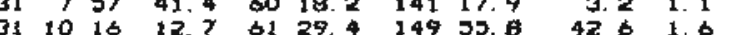
$\begin{array}{lllllllllll}31 & 10 & 16 & 12.7 & \text { b1 } & 29.4 & 149 & 55.6 & 42.6 & 1.6 & 5 \\ 31 & 13 & 57 & 53.9 & 61 & 7.3 & 149 & 30.8 & 44.3 & 1.8 & 15\end{array}$ $2 \quad 155$ $94 \quad 0.1 \mathrm{~B}$ 45 o. 25 a 12.74 63 0.24

$70 \quad 0.47$ 74 0. 32 $\begin{array}{lll}81 & 0 & 12 \\ 63 & 0.99\end{array}$ 73 0.17

$\begin{array}{ll}78 & 0.25 \\ 59 & 0.15\end{array}$ 45 0. 18 7 . 0.11 37 0. 36 $020.11 \quad 10.8$ $\begin{array}{ll}71 & 0.12 \\ 78 & 0.21\end{array}$ $\begin{array}{ll}1 & 0.12 \\ 0 & 0.21\end{array}$

$73 \quad 0.19$ $\begin{array}{ll}9.9 .40 \\ 0.6 & 0.07\end{array}$ 56 0. 62 as 0.12

$5 \quad 155 \quad 80 \quad 0.19$ $6 \quad 1351160.53$ $\begin{array}{rrrr}2 & 164 & 86 & 0.10 \\ 3 & 193 & 157 & 0.29\end{array}$ $75 \quad 0.94$ $46 \quad 0.58$ $\begin{array}{ll}73 & 0.06 \\ 80 & 0.21\end{array}$ 370.63

49 o. $2 t$

57 0. 37

430.34 $\begin{array}{ll}72 & 0.22 \\ 84 & 0.05\end{array}$ $89 \quad 0.74$

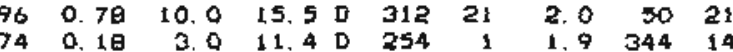
$34414 \quad 1.0 \quad 160$ 76 11.

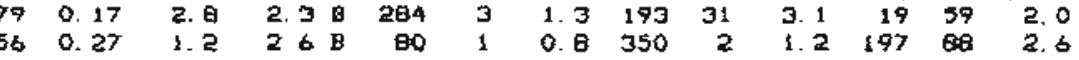


ORICIN TINE UAT N LOHE H DEPTH MUC ND NG GAP DO RES

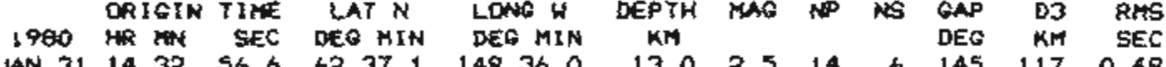
JAN $31 \quad 1432 \quad \$ 6.6 \quad 6237.1 \quad 14936.0$ 1736 20. 41 br. 14926. 71250241 61 39.31433 .8

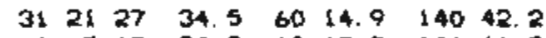

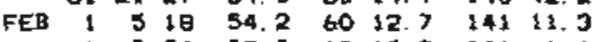

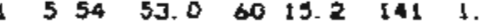

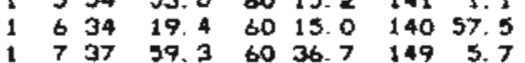

$1 \quad 8 \quad 32 \quad 27.4 \quad 6125.7 \quad 14722.8$ $1920 \quad 49.7$ 61 30.415017 .6

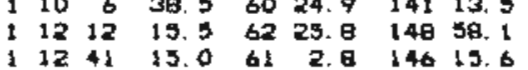

1132132.56013 .114113 .6

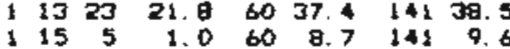

118251.060 6. 19147.4

₹20 12 30. 8 60 $17.2 \quad 14023.5$

$2213 \quad 26.5$ b1 44.5 149 46.5

242931.7 60 9.4 141 26.5

$\begin{array}{rrrrrrr}\text { 13. } 0 & 2.5 & 14 & 4 & 145 & 117 & 0.49 \\ 34 & 1.9 & 16 & 3 & 179 & 76 & 0.40\end{array}$

$39.51 .3 \quad 9 \quad 6 \quad 176 \quad 440.55$

16. $1.25 \%$ i 53 st 0.24

17. $3 \quad 1.9 \quad$ o $4 \quad 142 \quad 87 \quad 0.06$

$4.61 .7 \quad$ b 114 51 0.34 $\begin{array}{rrrrrrr}7.1 & 0.9 & 3 & 2 & 166 & 114 & 0.16 \\ 10.7 & 1.5 & 7 & 3 & 129 & 11 & 0.20\end{array}$

18. $4 \quad 1.7 \quad 19 \quad 3 \quad 135$

$61 \quad 0.53$

$\begin{array}{llllll}1.7 & 11 & 4 & 123 & 84 & 0.20\end{array}$

\section{1.7}

$4 \quad 112$

78 O. 18

$\begin{array}{rllllll}7.2 & 1.3 & 5 & 2 & 165 & 69 & 0.13\end{array}$

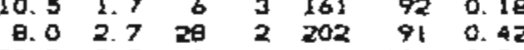

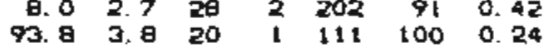

17. $02.0 \quad 6 \quad 1 \quad 166 \quad 890.17$

$\begin{array}{lllllll}40.2 & 1.8 & 15 & 9 & 156 & 57 & 0.28\end{array}$

4 ML EMRC

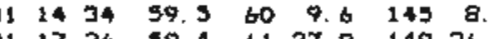

$\begin{array}{lllllll}10.0 & 1.5 & 7 & 3 & 129 & 91 & 0.20 \\ 20 & 1.0 & 7 & 5 & 143 & 50 & 0.47\end{array}$

$\begin{array}{rrrrrrr}29.9 & 1.6 & 7 & 3 & 126 & 62 & 0.15 \\ 19 & 2.2 & 13 & 215 & 108 & 0.53\end{array}$

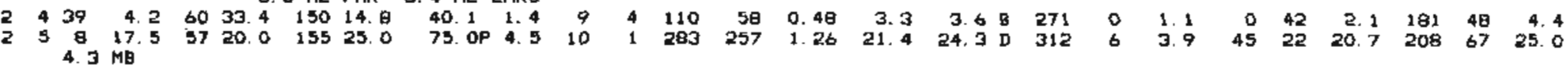

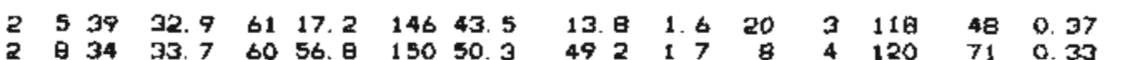

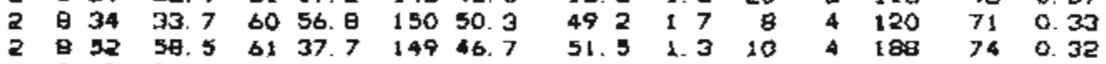

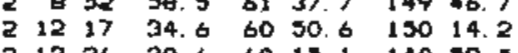

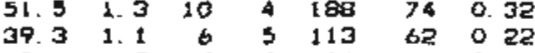

$\begin{array}{llllllll}2 & 13 & 45 & 49.7 & 62 & 49.1 & 148 & 10.7 \\ 2 & 14 & 31 & 13.3 & 50 & 55.0 & 139 & 53.6\end{array}$

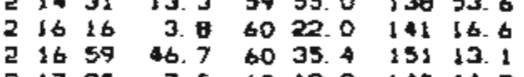

5. 92.2 16

$\begin{array}{rrr}5.9 & 2.2 & 16 \\ 29.3 & 1.0 & 3\end{array}$

$\begin{array}{llll}113 & 62 & 0 & 22 \\ 128 & 02 & 0.04\end{array}$

5261.4

$324130 \quad 0.55$

$\begin{array}{rrrrrrrrr}5 & 246 & 130 & 0.55 & 4.9 & 3.3 & 16 & 160 & 30 \\ 2 & 263 & 52 & 0.07 & 12.5 & 23.2 & 0 & 139 & 6 \\ 2 & 119 & 67 & 0.23 & 1.2 & 3.0 & 8 & 347 & \\ 5 & 124 & 00 & 0.39 & 2.5 & 7.0 & 1.37 & 187 & \end{array}$

$\begin{array}{llllllll}2 & 1725 & 7.5 & 60 & 18.9 & 140 & 44.2\end{array}$

44. 2 2.1 1

$\begin{array}{llllllll}2 & 19 & 3 & 16.2 & \text { bl } & 51.1 & 150 & 9 \\ 2 & 20 & 46 & 53.9 & \text { d } & 25.6 & 146 & 30\end{array}$

2 $21 \quad 1$ 5日. 0 क2 19.9 14957.2

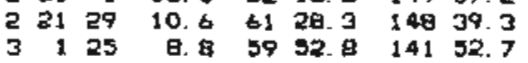

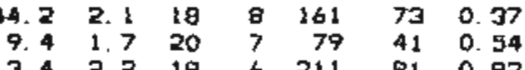

$\begin{array}{rrr}13.4 & 2.2 & 19 \\ 30.2 & 2.1 & 22 \\ 0.9 & 0.5 & 4\end{array}$

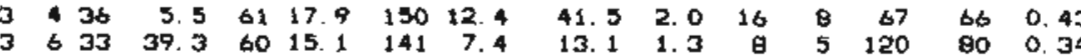

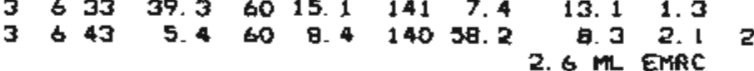

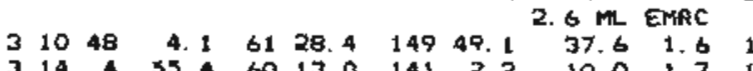

ERZ O AZ1 DIPL BEI ALZ DIPZ SEZ ALZ DIPJ EE3

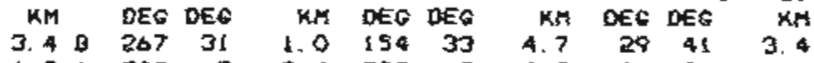

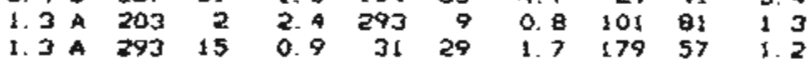

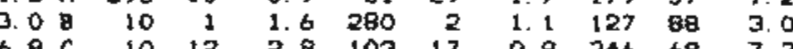

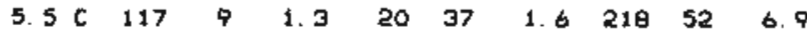

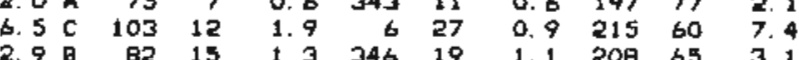

$\begin{array}{lllllllllll}\text { t. } 6 \text { A } & 203 & 18 & 0.6 & 16 & 14 & 1.2 & 156 & 72 & 17 \\ 4.9 \text { G } & 101 & 1 & 0.9 & 191 & 4 & 1.5 & 357 & 06 & 4 & 7\end{array}$

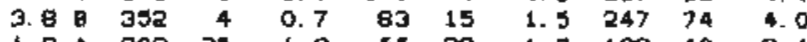

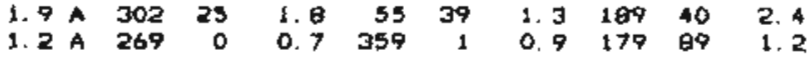

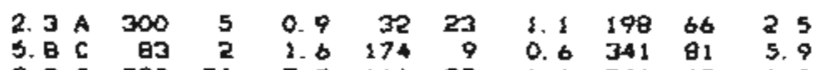

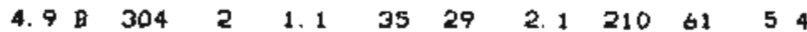

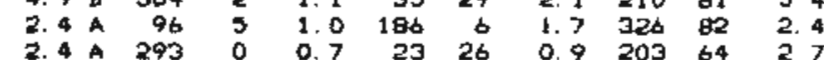

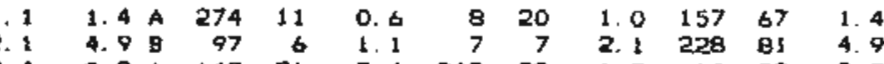

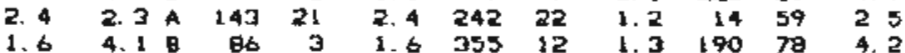

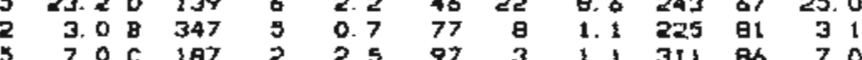

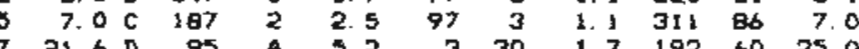

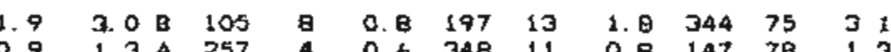

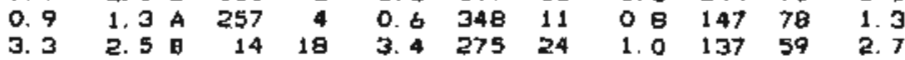

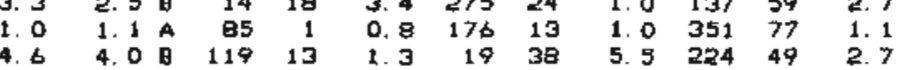

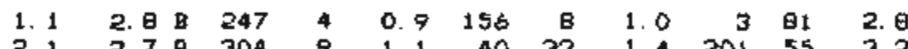

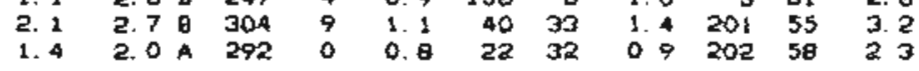
$\begin{array}{lllllllllll}5 & 1.4 A & 117 & 24 & 1.0 & 9 & 35 & 1.6 & 233 & 45 & 1.4\end{array}$

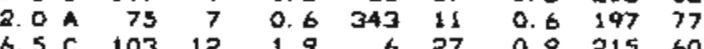

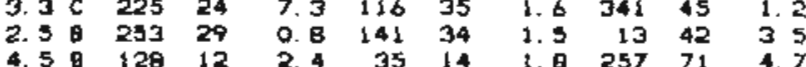


ORIGIN JIME LAT N LONG W DEPTH MAO NP NS GAP 03 RHS 1960 3 16 7 43. 9 60 12.4 141 1.

$328 \quad 5 \quad 27.4$ 63 49 \& 14854.5

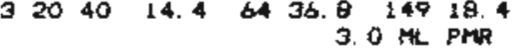

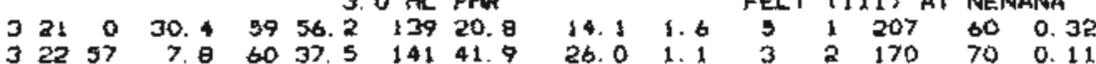
G. 127,2 OEC KH SEC $\begin{array}{lllllll}92.4 & 3.7 & 13 & 3 & 171 & 236 & 0.50\end{array}$ 14. FELT IIIIY AT NENANA

ERH ERI O AZ1 DIPI SEI AZE DIP2 EEZ AZJ DIPS SEO

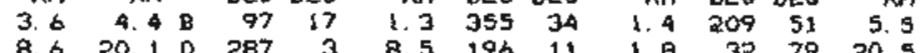

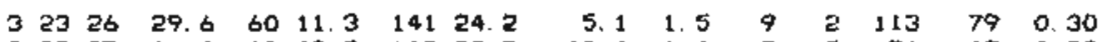

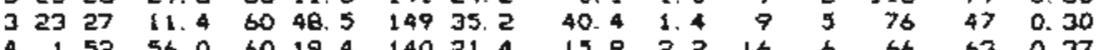

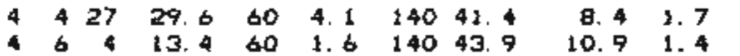

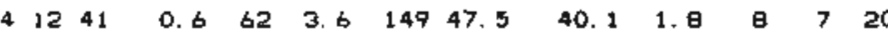

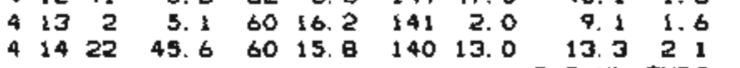

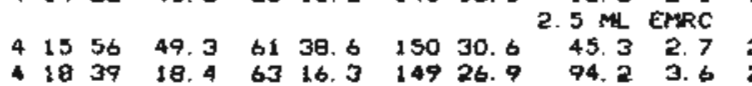

$423755.96050 .8 \quad 14939.4$ $504721 .+6010.514113$. 5 a 24 o. 3 b2 15.9 149 12.6

$\begin{array}{llllllll}5 & 10 & 13 & 19.9 & \text { b. } & 10.3 & 147 & 55.6\end{array}$ $\begin{array}{rrrrrrrr}5 & 10 & 30 & 40.8 & 62 & 5.0 & 147 & 52.5 \\ 5 & 10 & 40 & 42.0 & 60 & 15.7 & 141 & 9.5\end{array}$ $51045 \quad 18.6 \quad 62 \quad 3.0 \quad 14755.1$

$\begin{array}{rrrrrrrrrr}34.1 & 1.5 & 12 & 7 & 90 & 57 & 0.35 & 1.6 & 2.2 & 297 \\ 15.0 & 0.9 & 4 & 3 & 572 & 98 & 0.23 & 19.1 & 16.30 & 75\end{array}$

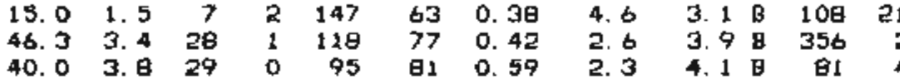

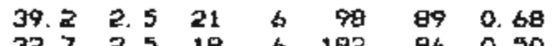

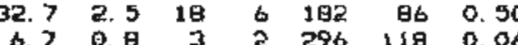
अ. 3 2.6 $20 \quad 5 \quad 182$ 80 0.53 $\begin{array}{llll}2.2 & 7.9 & C & 165 \\ 2.4 & 1.9 & \text { A } & 344\end{array}$ $4.6 \quad 418$ 26 5. 326 30 25.0 95 49 250 $\begin{array}{lllllll}1.2 & 225 & 12 & 52 & 69 & 77 & 2.0 \\ 1.4 & 244 & 14 & 3.5 & 44 & 75 & 25.0\end{array}$ $\begin{array}{llllllll}3 & 0.7 & 352 & 16 & 1.0 & 103 & 74 & 2.7\end{array}$ 0.8306 19 1.2 168 66 3.3 $\begin{array}{lllllll}0.9 & 207 & 34 & 4.9 & 18 & 56 & 1.9 \\ 0.7 & 202 & 22 & 3.3 & 357 & 66 & 1.9\end{array}$ $\begin{array}{lllllll}1.2 & 357 & 32 & 1.6 & 196 & 57 & 5.6\end{array}$

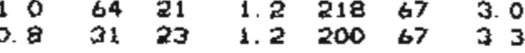
$\begin{array}{rrrrrrr}0.8 & 197 & 15 & 1.1 & 345 & 72 & 3.3 \\ 23 & 111 & 3 & 3.5 & 291 & 87 & 20.5\end{array}$ $\begin{array}{lllllll}0.9 & 329 & 29 & 1.3 & 142 & \text { bl } & \text { 2. } 4\end{array}$ $\begin{array}{lllllll}2.4 & 321 & 35 & 1.1 & 194 & 40 & 25.0\end{array}$ 2.6 265 3 1.3 119 bs 3.9 0.92032310 ab 3.9 $\begin{array}{lllllll}2.1 & 75 & 5 & 1.0 & 277 & 05 & 7.0\end{array}$

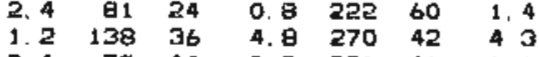
$51324 \quad 4356024.014118$.

$51452 \quad 45.9 \quad 60 \quad 14.9 \quad 14046$. 51546 2. 2 b2 $21.4 \quad 14930.1$ $518 \quad 7 \quad 27.9$ 60 15.6 140 12. 9

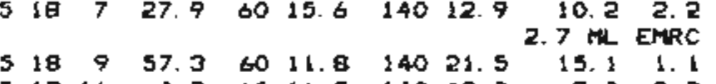
\begin{tabular}{lllllll}
$12 t$ & 63 & 0.34 & 1.9 & $4.3 \mathrm{~B}$ & 318 \\
\hline
\end{tabular} 518 16 4.9 to 16.5 140 4 a. $\begin{array}{lllllll}51924 & 36.3 & \text { to } 4.8 & 141 & 4.6\end{array}$ $647 \quad 159$ 60 $17.0 \quad 141 \quad 16.5$ b 99 15. 3 61 39.1 149 44.2 3. 8 1.5

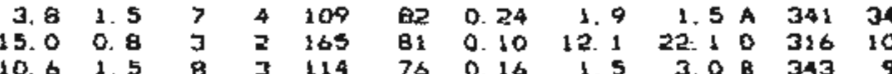

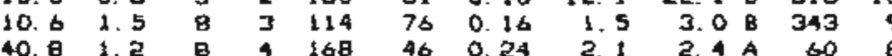
54531.3 60 12.3 14121.6 5.0 1.0

$\begin{array}{rrrrrrrr}6 & 0 & 24.6 & 00 & 17.5 & 141 & 16.3 \\ 6 & 10 & 20 & 17.1 & \text { al } & 32.4 & 150 & 13.1\end{array}$ $6 \quad 1344$ 56. 0 b0 39.9 131 55 . 5 $\begin{array}{llllllll}6 & 14 & 7 & 21.7 & 60 & 16.9 & 141 & 17.2 \\ 6 & 15 & 0 & 21.5 & 60 & 27.5 & 147 & 37.7\end{array}$ b $1720 \quad 43.6$ bo 67.0 141 3.5 $6173936.35951 .8 \quad 15151$.

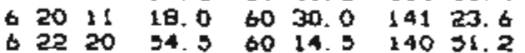

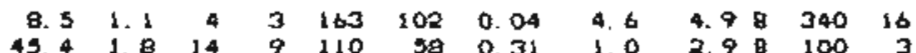

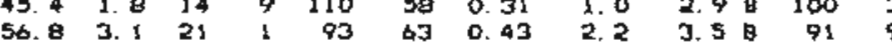

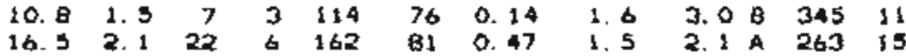

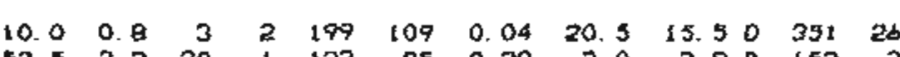

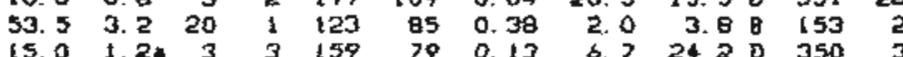
7214 o. 9 b: $37,3 \quad 147 \quad 42$ $\begin{array}{lllllll}2.4 & 79 & 44 & 0.9 & 251 & 46 & 1.6 \\ 1.1 & 49 & 14 & 1.4 & 212 & 75 & 4.4\end{array}$

$\begin{array}{llllllll}0.9 & 32 & 21 & 1 & 3 & 189 & 67 & 2.6\end{array}$

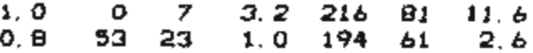
$\begin{array}{rrrrrrr}9.3 & 224 & 11 & 4.5 & 84 & 76 & 1.9 \\ 0.9 & 26 & 73 & 1.5 & 193 & 56 & 4.1\end{array}$ $\begin{array}{lllllll}1.1 & 223 & 35 & 2.3 & 101 & 37 & 0.9\end{array}$ $\begin{array}{lllllll}6.7 & 51 & 26 & 1.7 & 207 & 62 & 25.0 \\ 0.7 & 75 & 17 & 1.2 & 206 & 71 & 3 .\end{array}$ $\begin{array}{rrrrrrr}0.7 & 75 & 17 & 1.2 & 226 & 71 & 3 \\ 1.3 & 325 & 34 & 1.7 & 162 & 35 & 2.7\end{array}$

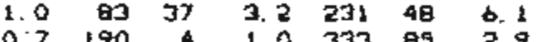
$\begin{array}{rrrrrrr}0.7 & 190 & 4 & 1.2 & 373 & 85 & 2.9\end{array}$ $\begin{array}{rrrrrrr}0.7 & 79 & 17 & 1.3 & 223 & 70 & 3.1 \\ 9.7 & 167 & 22 & 1.3 & 25 & 63 & 2.3\end{array}$ 1. $\begin{array}{llllllll}3 & 102 & 3 \text { b } & 25.0 & 234 & 43 & 7.0\end{array}$ $\begin{array}{rrrrrrr}2.0 & 43 & 3 & 1.2 & 277 & 86 & 3.9 \\ 0.8 & 259 & 14 & 2.6 & 92 & 76 & 28.0\end{array}$

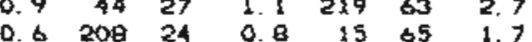




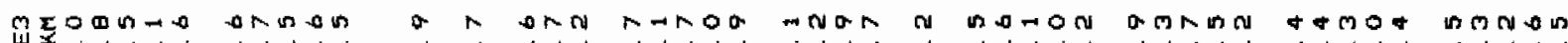

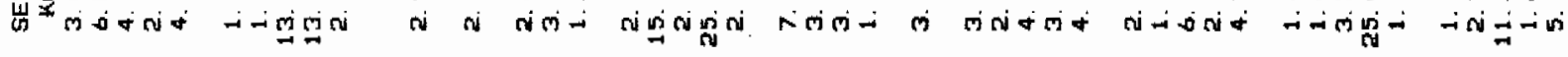

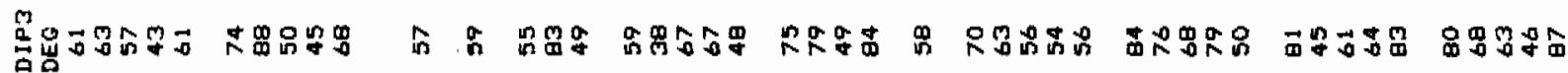

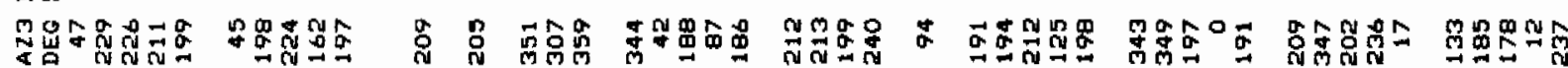

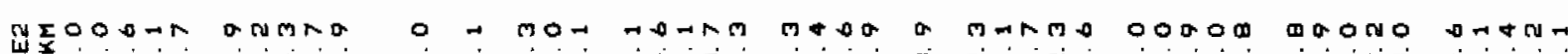

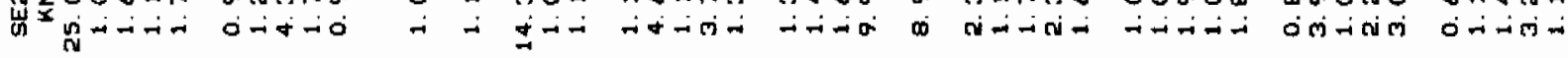

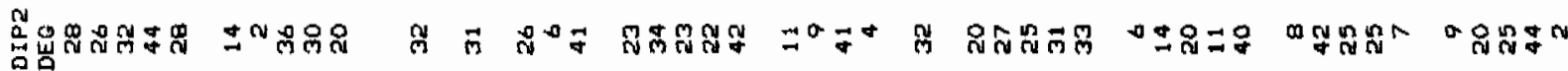

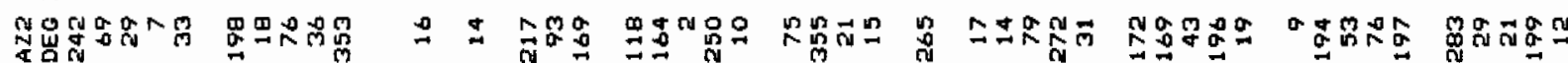

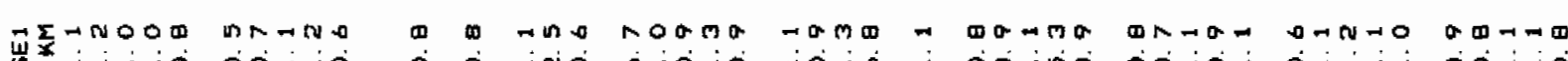

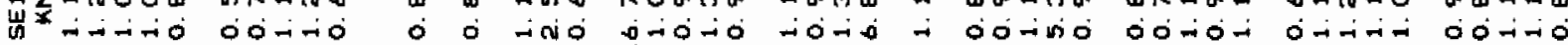

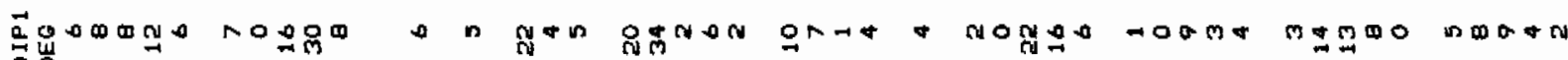
员岩

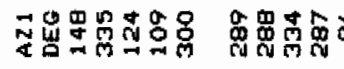

$0000 \times 90004$ NEmOODH Un UNM

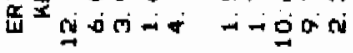

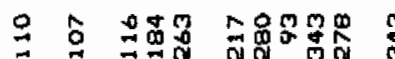

THलm

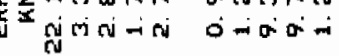

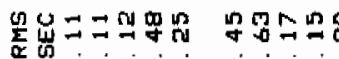

íód ócó

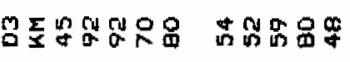

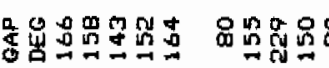

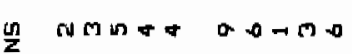

\section{$\Delta \quad \& \quad \cos 4$}

i

o日

on लू

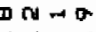

فे

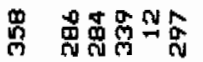

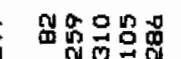

gas

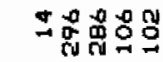

d $\cos$ a

$\infty \times m 0$

m $40<$ 向

ரिष

$0 \mathrm{~m}$

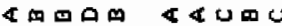

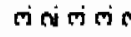

ni 10 im

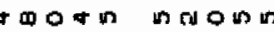

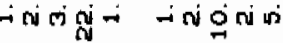

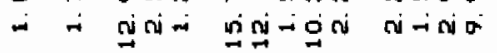

in $0 \mathrm{nos}$,

$00=0$

mono On m n m

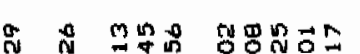

o D 0 c d

(⿻)

00000

ó 0 i

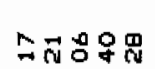

का $\rightarrow$ म

i - in

$2000 M N 000$

\section{+}

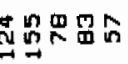

$\sin \frac{\sin }{\sin }$

o óóó

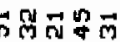

onरำ

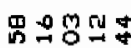

0000

ó ó

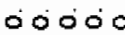

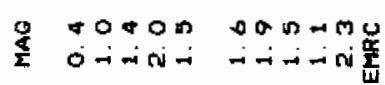

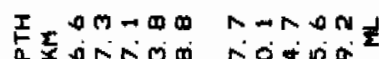

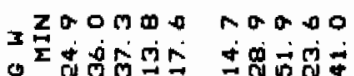

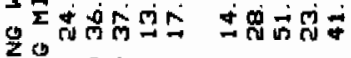

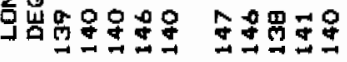

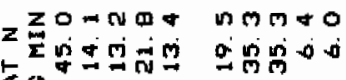

$\rightarrow 0$ ता व

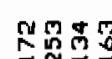

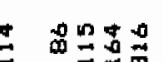

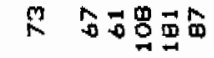

ang

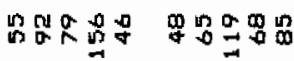

\section{$\therefore \quad$ in}

तथ

प Mm

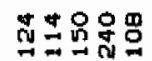

罗睓过

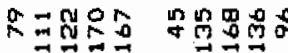

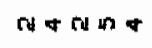

$\infty 0 \rightarrow 0$

ond

co $0 \mathrm{~N}+$

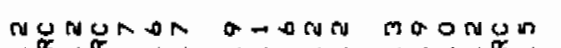

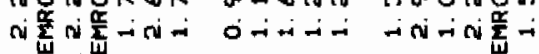

s

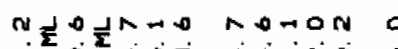

$00+\frac{1}{10}$

$-i \cos -$

19 -100

$\rightarrow \operatorname{lng} 0$

$m=m M$

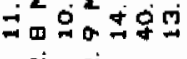

ni ni

a 100

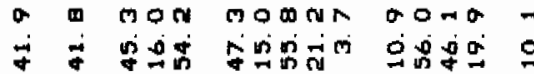

ㅇㅇㅇㅇㅇㅇㅛ

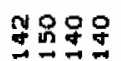

$\omega=0+0$

(ข $\rightarrow$ -

No 0 T

$\ln n+0$

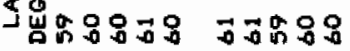

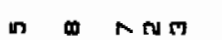

\& क क्षं

$m=n$

$00-n$

or in

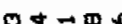

लं

$\therefore$ -

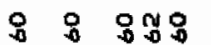

$9020=$

กู่

00004

में

o.oom

monot

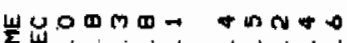

0

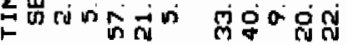

$\Rightarrow$ 我的

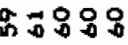

웅요용

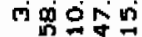

cond $m$

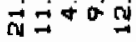

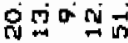

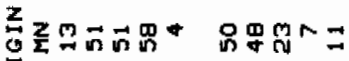

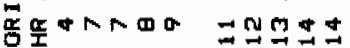

$\pm$

तुल

ongy

oom

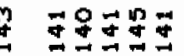

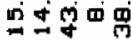

(4) का 0

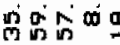

mom 0

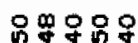

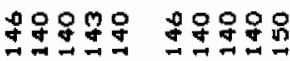

MAMRN MNRMR

a然需

$00 n \rightarrow \infty$

$\tan 00$

oteot gano

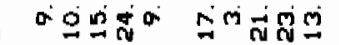

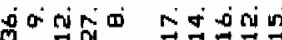
兽

$\rightarrow$

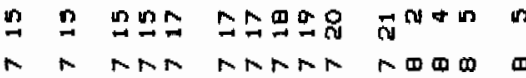

四田
웅

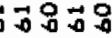

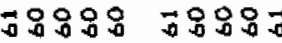

MNOA MMMNM

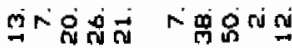

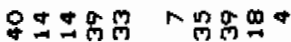

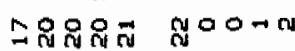

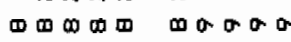


SOUTHEAN ALASHA EARTMOUAKES, JAN - MAR 1980

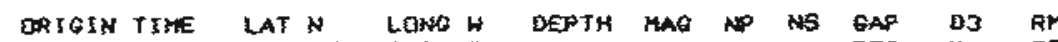

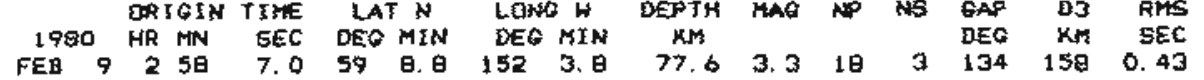

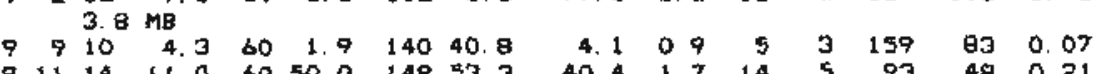

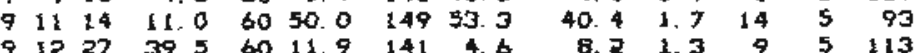

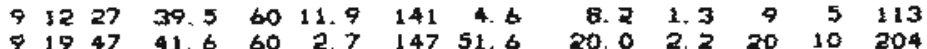

490.21

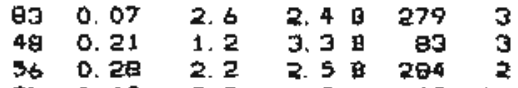

$\begin{array}{lllllll}921 & 19 & 44.5 & 61 & 45.2 & 149 & 42.3 \\ 922 & 26 & 13.5 & 39 & 21.5 & 139 & 9.8\end{array}$

$\begin{array}{lllllll}79.0 & 2.1 & 17 & 10 & 153 & 97 & 0.4\end{array}$

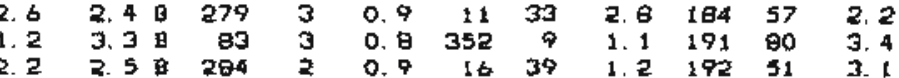

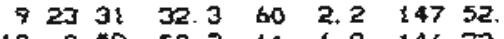

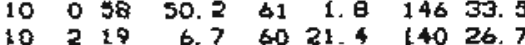

$\begin{array}{lllllllll}3 & 1 & 336 & 107 & 0.06\end{array}$

1.9 A 255 i6

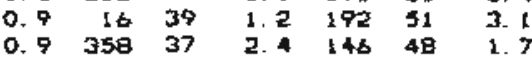
$\begin{array}{lllllllll}32 & 29.2 & \text { o1 } & 16.1 & 152 & 10.1 & 139.6 & 4.0 & 24\end{array}$

$\begin{array}{lllllllllllll}10 & 4 & 25 & 14.2 & 61 & 29.0 & 146 & 46.9 & 22.4 & 2.0 & 22 & 9 & 105\end{array}$

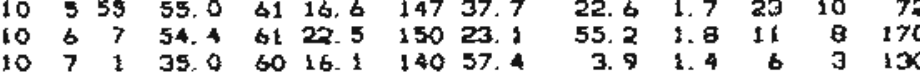

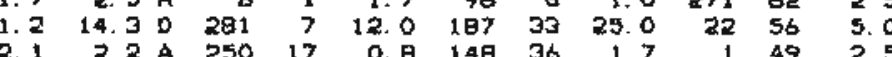

$\begin{array}{llrllllll}10 & 1394 & 5.5 & 59 & 50.5 & 44027.4 & 11.6 & 2.3 & 24\end{array}$ $1014 \quad 27$ 25. 6017.4 140 55.5 b. 1 1.

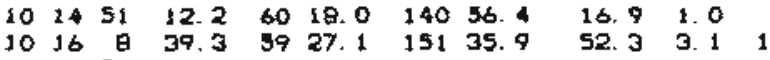
3. 39

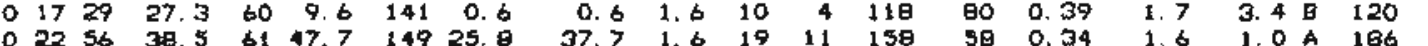

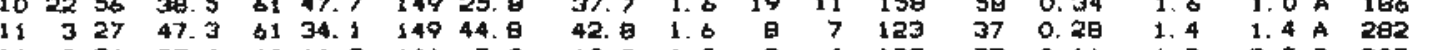

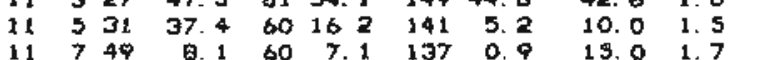

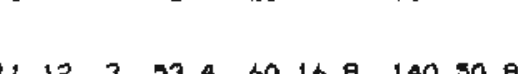
2. 4 ML EMRC

$\begin{array}{lllllllll}11 & 12 & 3 & 53.4 & 60 & 16.9 & 140 & 50.1 & 0\end{array}$ $\begin{array}{llllllll}11 & 12 & 5 & 45.5 & 65 & 34.7 & 149 & 50.9 \\ 11 & 16 & 3 & 57.9 & 60 & 31.2 & 145 & 7.3\end{array}$

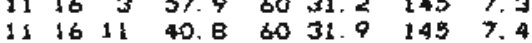
1119 16 45.3 60 32 日 141 10

1) $1921 \quad 7.96016 .614133 \mathrm{a}$ 112120345 60 15.6 140 59.6 112235 29.5 $6127.6 \quad 14918.2$

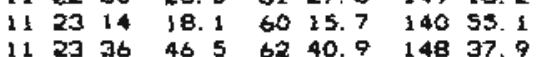

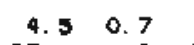

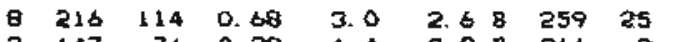
$\begin{array}{lllllll}147 & 76 & 0.39 & 1.4 & 3.6 & 316 & 316\end{array}$ $\begin{array}{lll}145 & 110 & 0.17\end{array}$ 5. 7 a.

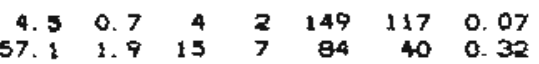

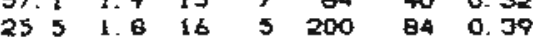
$\begin{array}{lllllll}24.9 & 1.8 & 17 & 7 & 189 & 84 & 0.39\end{array}$ 650.14 $\begin{array}{lllllll}2.6 & 1.1 & 4 & 3 & 125 & 77 & 0.29\end{array}$ 6.6 1.0 52127

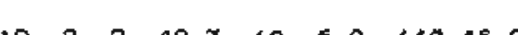
$\begin{array}{lllllll}1.8 & 0.7 & 4 & 2 & 159 & 108 & 0.06 \\ 29.5 & 2.3 & 11 & 5 & 267 & 132 & 0.52\end{array}$

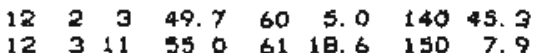
$12+31$ b.5 6340.19041 .7

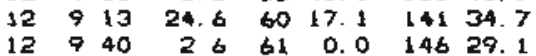
$\begin{array}{cccccccc}13.5 & 1.9 & b & 1 & 184 & 85 & 0 & 16 \\ 49.3 & 2.2 & 16 & 4 & 64 & 62 & 0 & 42\end{array}$ $\begin{array}{lllllll}\text { E7. } 6.4 \text { is } 1 & 153 & 229 & 0.57\end{array}$ $12940 \quad 26$ b1 0.0 14b 29.1 $\begin{array}{rrrrrrrrr}12 & 10 & 31 & 15 & 3 & 60 & 12.9 & 141 & 0.7 \\ 12 & 13 & 14 & 28.2 & 61 & 49.1 & 149 & 16.6\end{array}$ 322157 23. 6014.2141 16. 1301915.5 bi 10.3 14b 18.2

9.91 .919

10. 1.4 $\begin{array}{rrrrrrr}10.4 & 1.6 & 9 & 3 & 107 & 59 & 0.28\end{array}$ 9.219 20 61 a $\begin{array}{lllll}2.6 & 5.9 & 117 & 6 \\ 1.2 & 2.0 & 250 & 7 \\ 2.0 & 1.5 & 253 & 37\end{array}$ $\begin{array}{lllll}\text { 2. } & 1.5 & \text { A } & 321 & 27 \\ 2 . & 1.4 & \text { A } & 328 & 19\end{array}$ 2.011 .40176

$\begin{array}{lllr}\text { 1. } & 5.7 & \mathrm{C} & 64 \\ 2.9 & 6.5 & \mathrm{C} & 275\end{array}$ $\begin{array}{lllll}1.5 & 1.9 & A & 42 & B \\ \text { 2. } 1 & 6.1 & C & 116 & 5\end{array}$ 4. 7 4. A B $312 \quad 12$ 1. 2 3.0 $\quad 3$ a 344 $\begin{array}{rrrr}5.3 & 25.0 & 0 & 115 \\ 0.8 & 2.7 & 8 & 296\end{array}$

$152.2 A 104$

$\begin{array}{llll}1.1 & A & \\ 0.1 & 2.0 A & A\end{array}$

0. $1.3 \mathrm{~A}$ 24

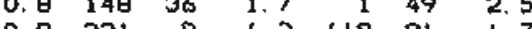

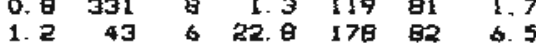

$\begin{array}{lllllll}1.7 & 145 & 15 & 2.3 & 208 & 71 & 5.2\end{array}$

$\begin{array}{rrrrrrr}0.9 & 278 & 5 & 0.6 & 98 & 68 & 1.9 \\ 1.1 & 275 & 10 & 0.6 & 73 & 76 & 1.5\end{array}$

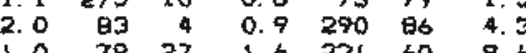
$\begin{array}{lllllll}0.9 & 9 & 35 & 3.3 & 142 & 45 & 2 . t\end{array}$ $\begin{array}{rllllll}0.9 & 47 & 11 & 1.2 & 216 & 79 & 3.9\end{array}$ $1.5 \quad 0904 \quad 1.7$ 222 $46 \quad 19.0$

$\begin{array}{lllllll}0.9 & 70 & 23 & 1.0 & 212 & 67 & 3.6 \\ 1.6 & 73 & 35 & 0.7 & 292 & 59 & 1.0\end{array}$ $\begin{array}{lrrrrrr}1.0 & 104 & 44 & 0.9 & 282 & 95 & 1.0 \\ 0.9 & 40 & 18 & 1.0 & 200 & 45 & 1.6\end{array}$ $\begin{array}{rrrrrrr}0.9 & 40 & 19 & 1.0 & 203 & 71 & 2.7 \\ 3.3 & 207 & 36 & 1.2 & 7 & 52 & 16.9\end{array}$

$\begin{array}{lllllll}2.0 & 24 & 26 & 1.0 & 222 & 68 & 6 .\end{array}$ $\begin{array}{rrrrrrr}0.7 & 59 & 12 & 1.2 & 10 & 76 & 2.0 \\ 2.2 & 70 & 72 & 0.9 & 200 & 46 & 1.4\end{array}$

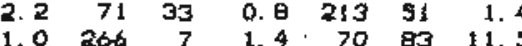
$\begin{array}{lllllll}0.1 & 334 & 7 & 0.7 & 162 & 93 & 5.7\end{array}$

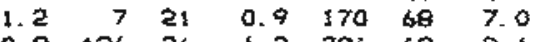
$\begin{array}{rrrrrrr}0.9 & 136 & 26 & 1.3 & 296 & 63 & 2.1 \\ 1.5 & 24 & 16 & 1.0 & 223 & 79 & 4.4\end{array}$ $\begin{array}{lllllll}1.4 & 215 & 27 & 4.7 & 64 & 50 & 4.4\end{array}$ $\begin{array}{lllllll}0.7 & 154 & 4 & 1.1 & 334 & 96 & 3.0\end{array}$ $\begin{array}{lllllll}0.3 & 05 & 2 & 2.6 & 358 & 60 & 25.0\end{array}$

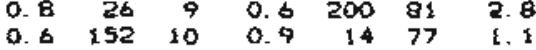
$\begin{array}{lllllll}0.7 & 10 & 26 & 1.1 & 212 & 62 & 2.4\end{array}$ $\begin{array}{rrrrrrr}0.8 & 273 & 19 & 0.5 & 106 & 70 & 1.2 \\ 0.6 & 1 & 15 & 1.0 & 207 & 73 & 2.1\end{array}$ $\begin{array}{lllllll}0.7 & 332 & 6 & 0.8 & 143 & 84 & 1\end{array}$ 
mEn

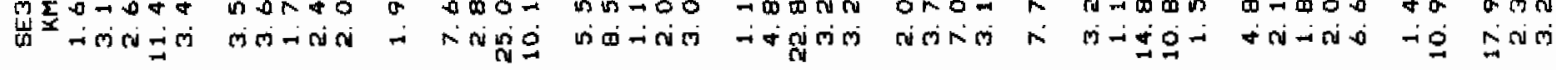

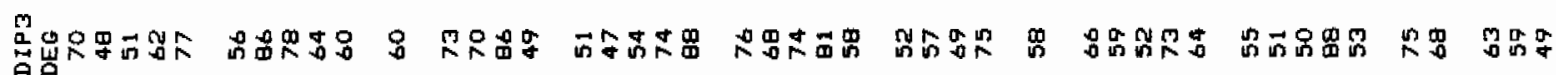

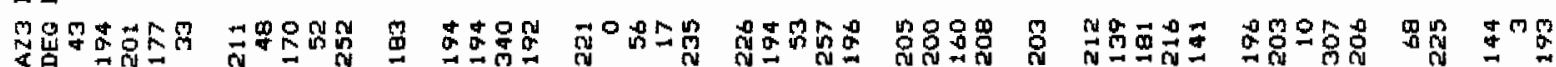

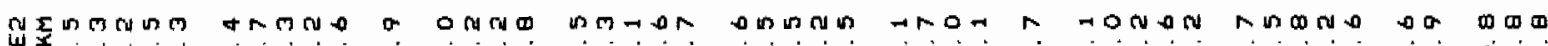

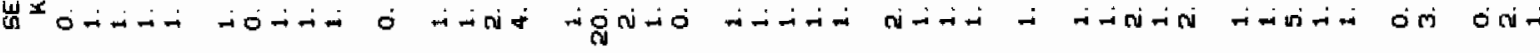

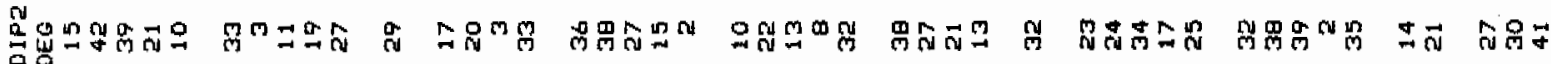

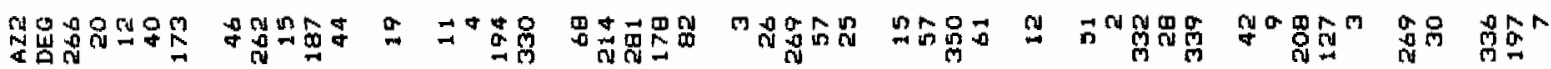

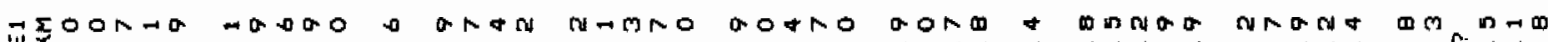

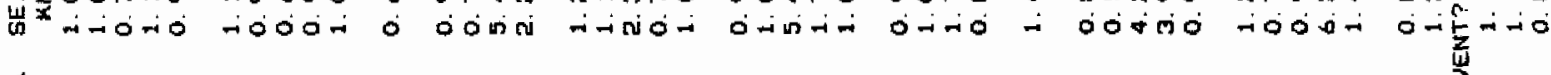

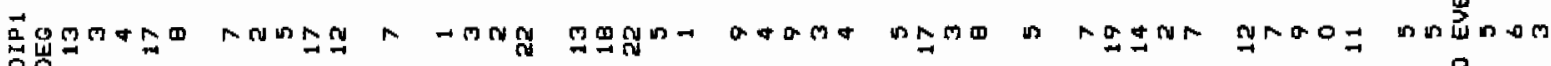

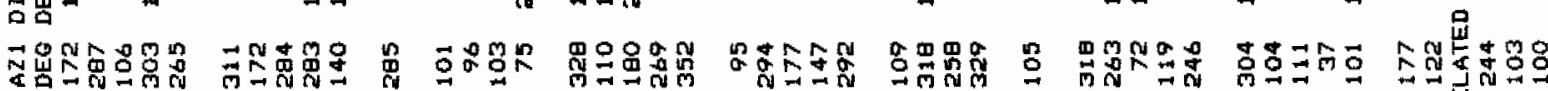
व

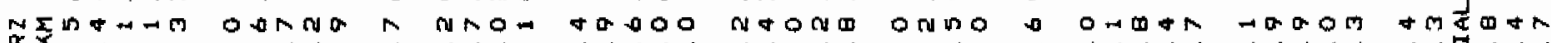
营

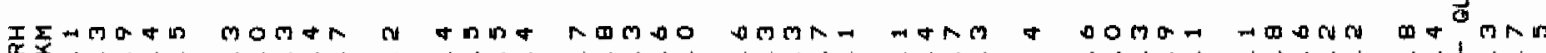
害

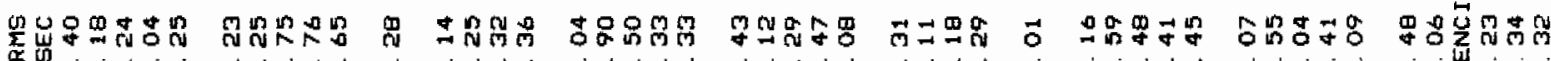

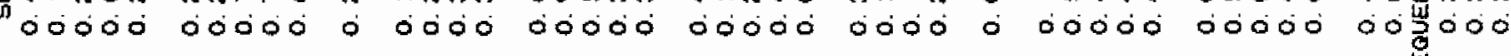

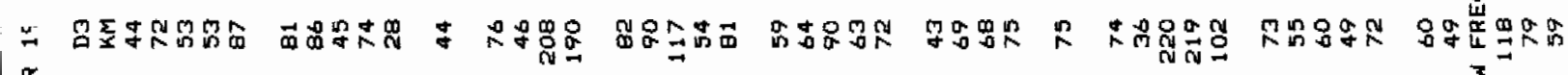

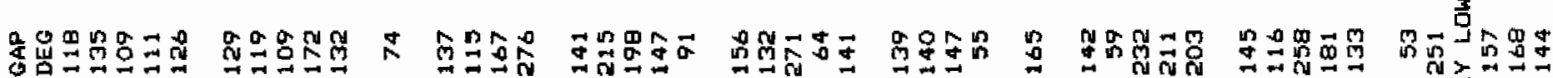

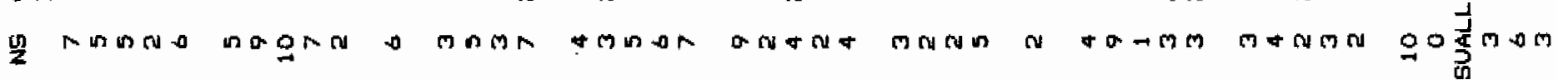

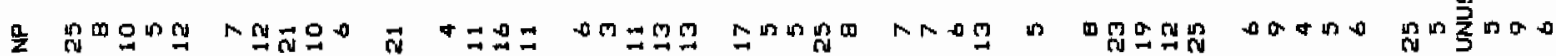

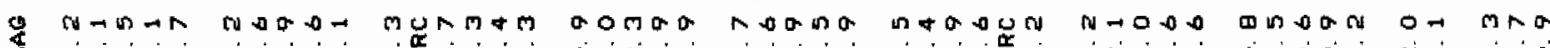

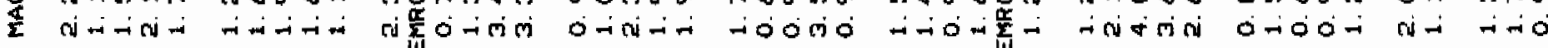

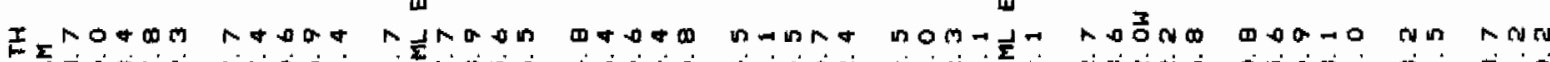

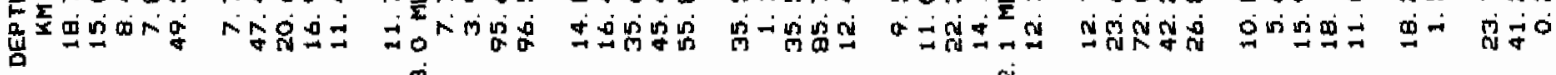

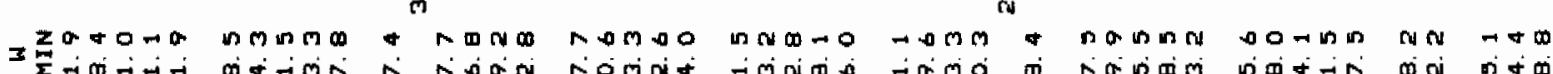

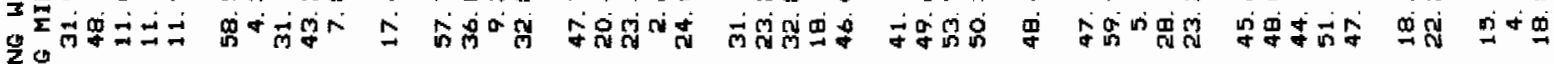

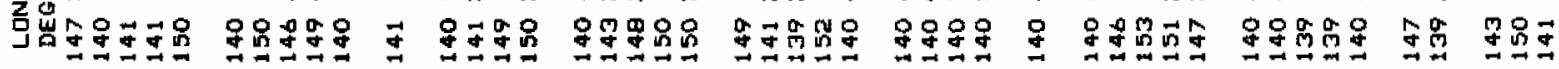

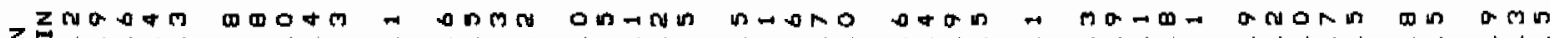

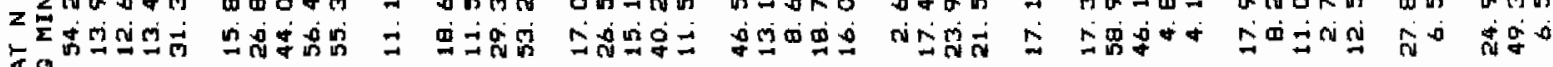

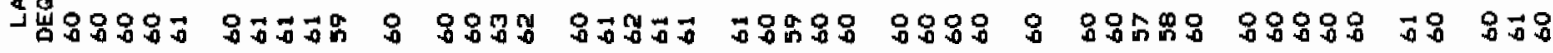

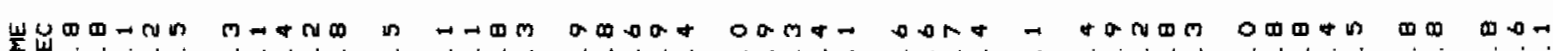
₹

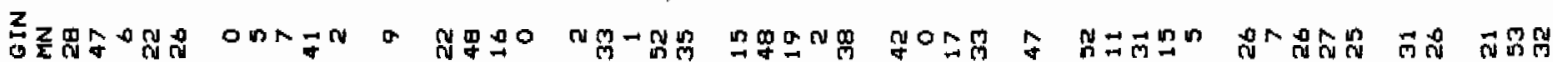

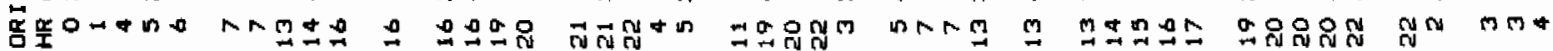

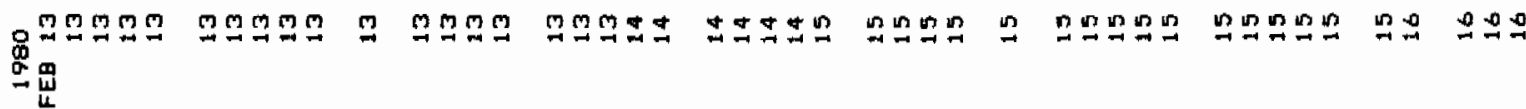




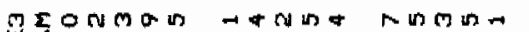

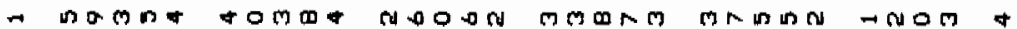

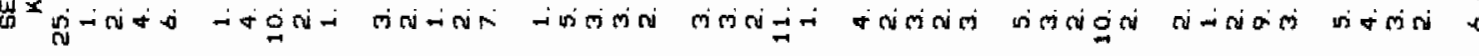

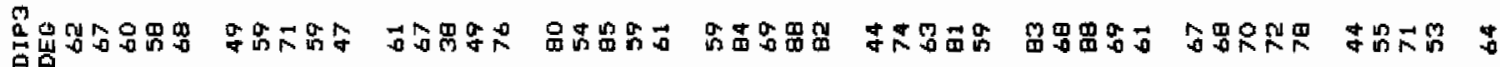

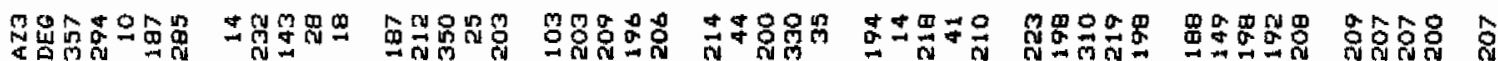

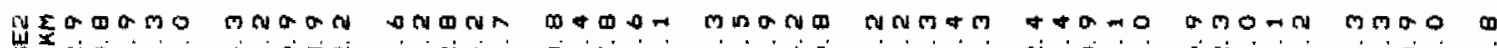

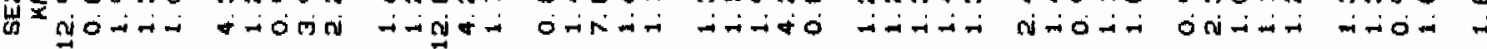

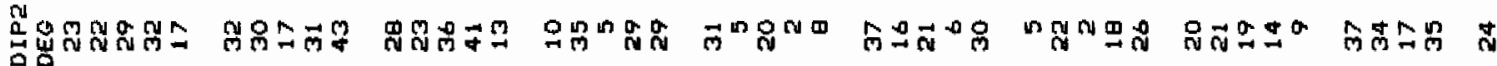

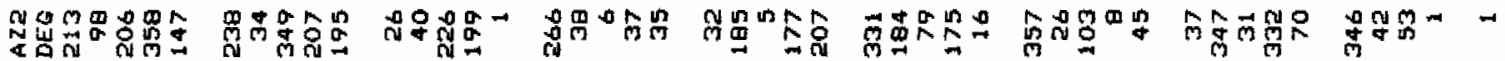

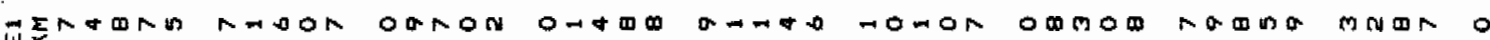

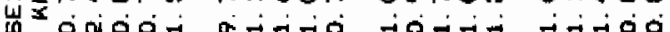

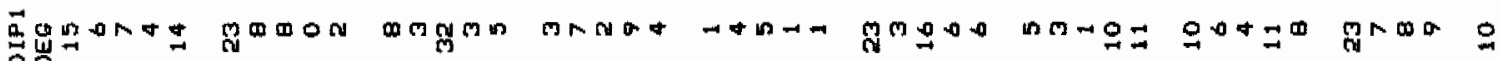

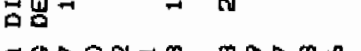

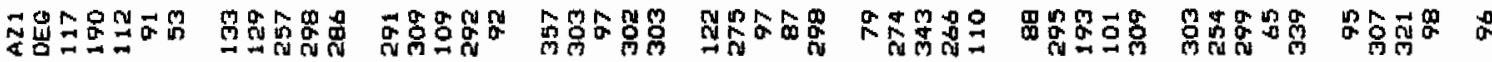

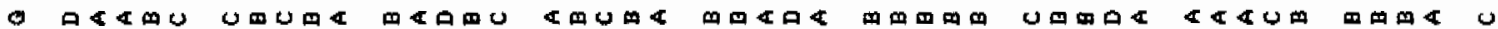

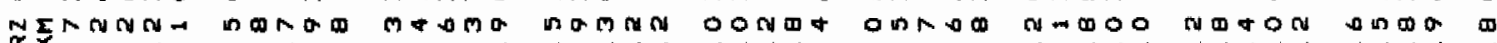
䒼

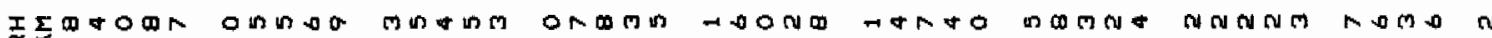
प्र

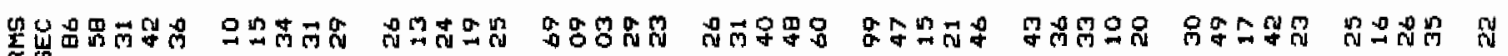

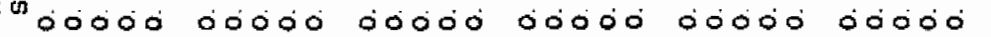
bióó ódió óó

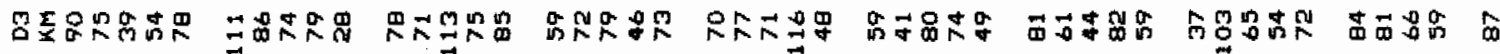

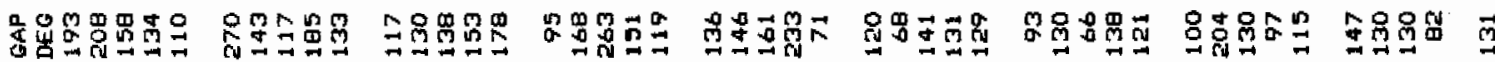

Q

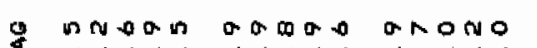

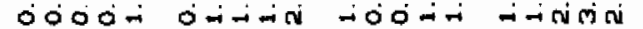

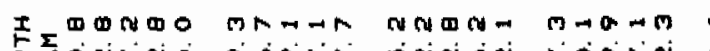

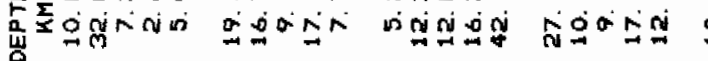
$+10+\mathrm{N}$ ơ

3 zom

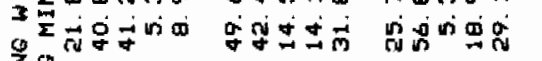

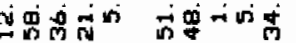

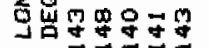
尘过王是是

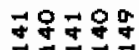

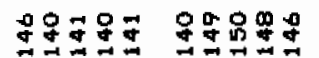

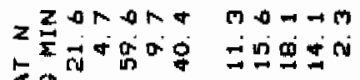

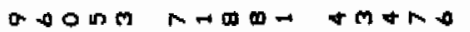

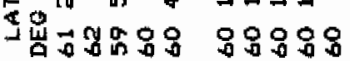
o w लें

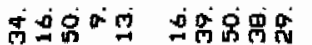

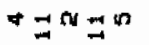
$\Delta+N M$ $m+m \infty$ an in o.

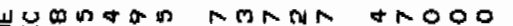
O\#政 manN MN 000 iं $\boldsymbol{\omega} \boldsymbol{\omega} \boldsymbol{\omega} \boldsymbol{0}$

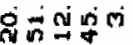

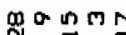
$+000$ an in $\Rightarrow$ ก 0 पं के

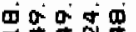

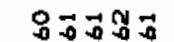
om $n \pi x$

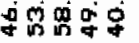
瑇星导是 on is 00 लं $\rightarrow \dot{0}-$

OMNM M 0. mamoo omमेंक O On n $g_{\rightarrow}^{\infty} \omega^{\frac{1}{2}}-$ a.onm notoun $0,0 \rightarrow m$ m

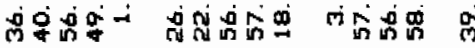

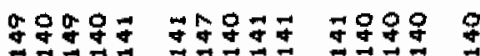

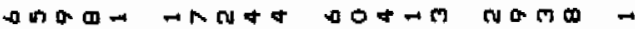

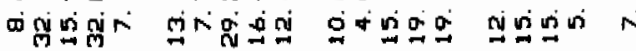

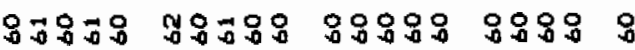

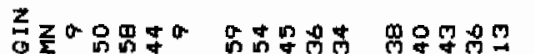

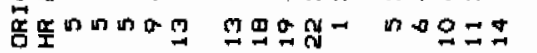

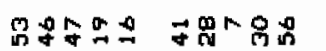

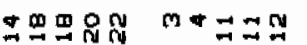

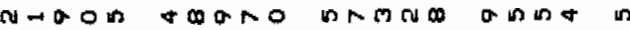

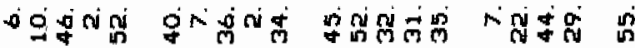

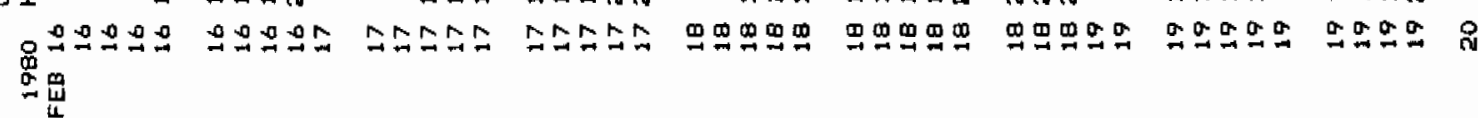




\section{ORIOIN JITE LAT $N$ LING $H$ DEPTH MAO NP NG OAP 03 gMS}

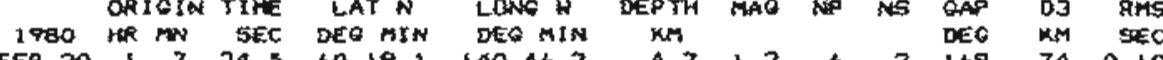

20 [ 7245 b0 16. 3 i 4046.3

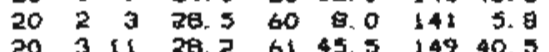

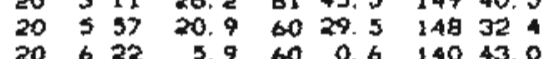

4.71 .2 o 2 169 74 o. 10

$\begin{array}{rrr}\text { B. } & 1.3 & 4 \\ 7.9 & 1.4 & 7\end{array}$

$\begin{array}{lll}138 & 106 & 0.12 \\ 161 & 59 & 0.67\end{array}$

207507.3 60 $19.1 \quad 141 \quad 17.7$

20 \&1 30.6 b1 24. 2 150 43.

$2010 \quad 39 \quad 23.0 \quad 6015.3 \quad 14056.0$

เ2. 2 1. 3

$\begin{array}{llll}142 & 66 & 0.46 \\ 154 & 0.35\end{array}$

$\begin{array}{lllllll}11.1 & 1.4 & 7 & 2 & 115 & 72 & 0.36 \\ 13.1 & 1.9 & 6 & 4 & 109 & 77 & 0.14\end{array}$

$\begin{array}{ccccccc}6.1 & 0.7 & 5 & 2 & 156 & 108 & 0.17\end{array}$

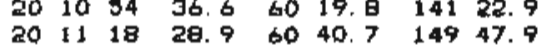

$\begin{array}{rrrrrrr}15.1 & 1.5 & 7 & 3 & 122 & 71 & 0.19 \\ 43.1 & 1.3 & 9 & 4 & 77 & 64 & 0.34\end{array}$

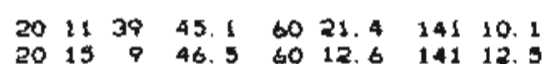
$\begin{array}{llllllll}20 & 15 & 9 & 46.5 & 60 & 12.6 & 141 & 12.5 \\ 20 & 16 & 9 & 56.7 & 59 & 49.6 & 141 & 36.5 \\ 20 & 16 & 26 & 7.4 & 60 & 7.7 & 140 & 56.3\end{array}$ $\begin{array}{rrrrrrrr}20 & 16 & 26 & 7.4 & 60 & 7.7 & 140 & 54.3 \\ 20 & 18 & 7 & 13.3 & 59 & 57.5 & 141 & 16.8\end{array}$

$11.7 \quad 0.9 \quad 5$

$\begin{array}{llll}2 & 144 & 96 & 0.28 \\ 2 & 195 & 90 & 0.37\end{array}$ $\begin{array}{lllllll}0.2 & 0.9 & 4 & 2 & 232 & 111 & 0.12 \\ 0.9 & 1.3 & 8 & 2 & 118 & 62 & 0.22\end{array}$

ERH ERZ O AZ1 OIP 16. 1 10. 5 D $\$ 14$ 18

1.1 1.2A 69 日

5. 3. ज.

$\begin{array}{llllr}\text { 1. } 7 & 2.8 & \text { B } & 335 & 14 \\ 1.6 & 3.1 & \text { B } & 369 & 0\end{array}$

2. 2 5.

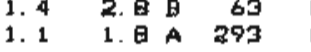

$\begin{array}{lllll}5.4 & \text { 6. } 4 & C & 333 & 13 \\ 1.4 & 3.4 & \text { B } & 290 & \text { ( }\end{array}$

$\begin{array}{rrrrr}1.0 & 3.4 & \theta & 200 & 7 \\ 3.0 & 4.0 & 0 & 84 & 2\end{array}$

$\begin{array}{lllll}3.0 & 5.1 & C & 104 & 9 \\ 1.0 & 1.0 & A & 311 & 17\end{array}$

$\begin{array}{lllllll}20 & 28 & 21 & 30.4 & 57 & 7.1 & 15638.4\end{array}$

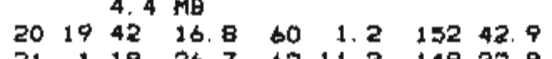

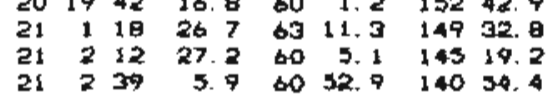

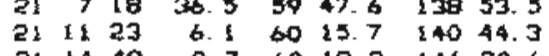
$21 \quad 14 \quad 49 \quad 0.7$ 60 $19.3 \quad 141$ 20. 6 $\begin{array}{llrrrrrr}21 & 16 & 42 & 21.4 & 60 & 4.3 & 139 & 46.6 \\ 21 & 18 & 7 & 33.3 & 60 & 12.1 & 139 & 33.0\end{array}$

90.005 .0

$\begin{array}{lllllllll}2 & 293 & 317 & 0.36 & 22.7 & 25.0 & 0 & 319 & 3\end{array}$

$96.7 \quad 3.5 \quad 15$ $\begin{array}{lll}157 & 119 & 0.36\end{array}$ $\begin{array}{rrr}18.5 & 0.6 \\ 5.1 & 1.2 & 3\end{array}$

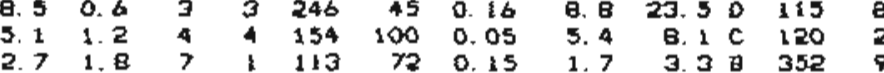

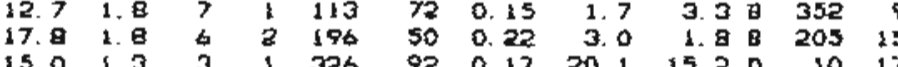
21 $1823 \quad 2.4 \quad 6313.7 \quad 44939.8$

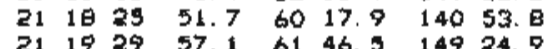
$\begin{array}{rrrrrrrr}21 & 19 & 29 & 57.1 & 61 & 46.5 & 149 & 24.9 \\ 21 & 23 & 1 & 24.6 & 62 & 56.1 & 149 & 3.6\end{array}$ $\begin{array}{llllllll}21 & 23 & 1 & 24.6 & 62 & 56.1 & 149 & 3.6 \\ 22 & 0 & 26 & 56.2 & 60 & 25.8 & 140 & 6.4\end{array}$

$\begin{array}{llllllll}22 & 0 & 35 & 57.9 & 61 & 44.7 & 149 & 39.4\end{array}$

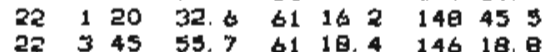
32422 52. 5 60 31.5 i41 24.1 $\begin{array}{llllllll}22 & 6 & 42 & 57.2 & 61 & 43.3 & 149 & 40.3\end{array}$ $\begin{array}{llllllll}22 & 6 & 53 & 3.6 & 61 & 42.0 & 149 & 39.5 \\ 22 & 8 & 5 & 20.7 & 40 & 4.6 & 139 & 26.8\end{array}$ $22927 \quad 33.7 \quad 6029.9 \quad 14060.0$ $\begin{array}{llllllll}22 & 10 & 1 & 47.8 & 61 & 79.2 & 449 & 44.1 \\ 32 & 10 & 53 & 9.8 & 62 & 26.2 & 149 & 47\end{array}$

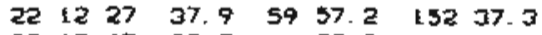
$\begin{array}{llllllll}22 & 12 & 47 & 27.7 & 61 & 53.2 & 149 & 15.9\end{array}$ $2213 \quad 297$ 60 16. 140468 $\begin{array}{rrrrrrrr}22 & 14 & 1 & 12.9 & 62 & 12.9 & 149 & 47.4 \\ 22 & 14 & 13 & 40.7 & 60 & 35.7 & 149 & 6.5\end{array}$

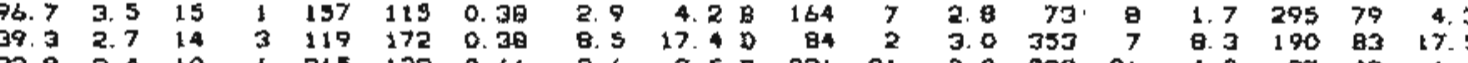

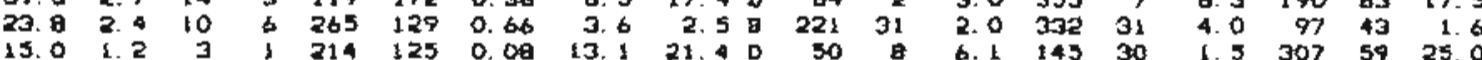
$\begin{array}{ccccccccccccccccccc}46.6 & 2.6 & 9 & 4 & 170 & 173 & 0.99 & 7.9 & 23.9 & 0 & 746 & 4 & 4.9 & 257 & 17 & 2.5 & 91 & 73 & 25.0 \\ 2.9 & 0.7 & 5 & 5 & 136 & 80 & 0.19 & 2.2 & 4.9 & 0 & 104 & 12 & 1.9 & 10 & 16 & 0.9 & 229 & 70 & 5.1\end{array}$

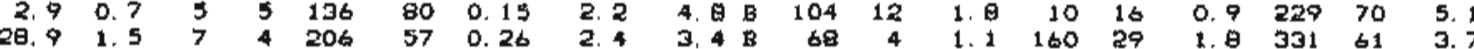

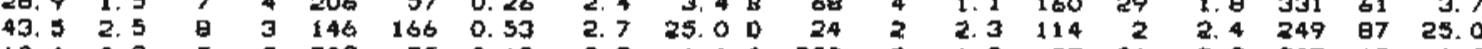

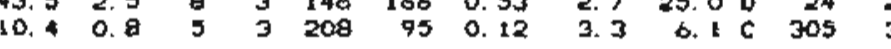

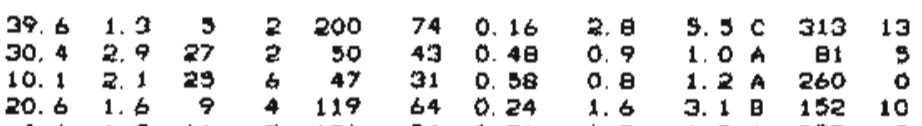
$\begin{array}{lll}2 & 37 & 21\end{array}$

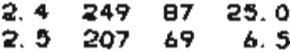

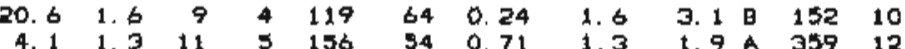
$\begin{array}{lllllll}2.0 & 46 & 19 & 1.5 & 191 & 67 & 5.9\end{array}$

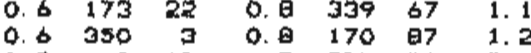

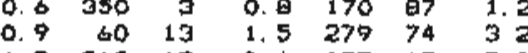

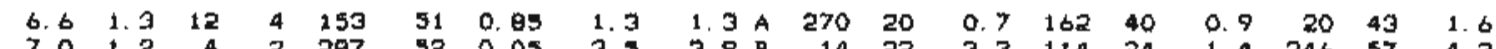

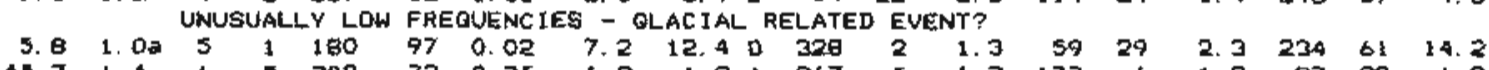

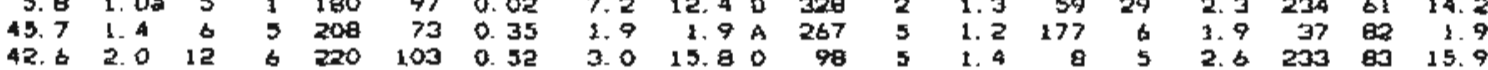

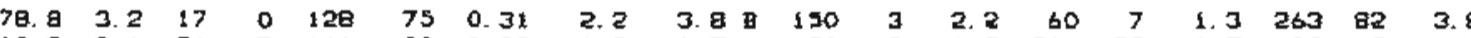

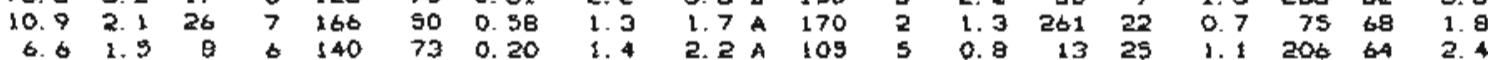

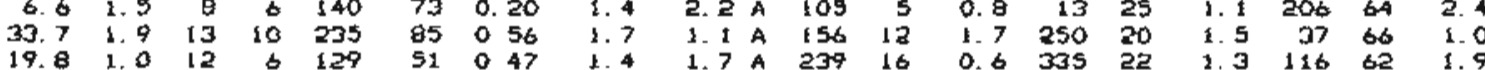


ORIGIN TIME LAT $M$ LONG W DEFTH MAG NP NS GAP DJ RMS

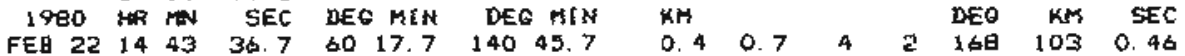

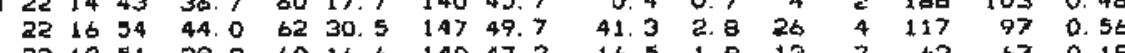

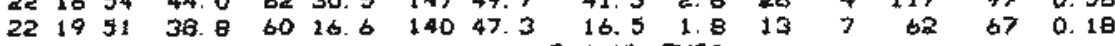

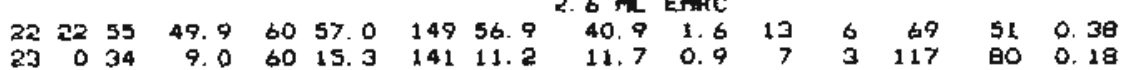
$23 \quad 0 \quad 54 \quad 509 \quad 6139.4 \quad 14943.5$ $\begin{array}{llllllll}23 & 3 & 21 & 11.4 & 60 & 1.9 & 142 & 21.9 \\ 23 & 3 & 43 & 19.2 & 62 & 17.9 & 149 & 34.6\end{array}$

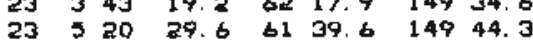

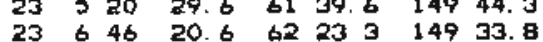

$237 \quad 7 \quad 5.4$ b0 $22.6 \quad 14019.4$

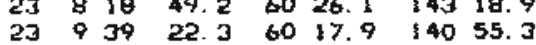
23115 7. 50 12 1 i41 13.2 $2312 \quad 5 \quad 283 \quad 5953.1 \quad 139$ 58.

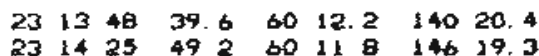

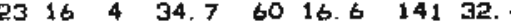

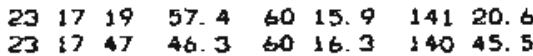

231 的 493.4 कर 24.7 14日 53.9

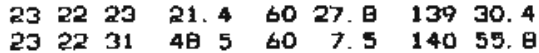

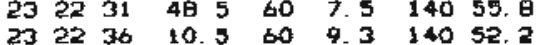

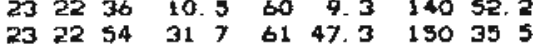

3. 1 ML. PMR

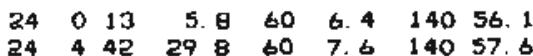

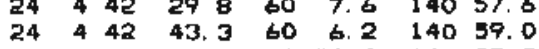

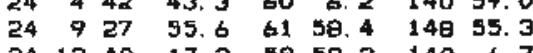
24124017.2 59 59.31406 .7 $\begin{array}{rrrrrrrr}24 & 14 & 33 & 0.5 & 61 & 46.7 & 131 & 5.1 \\ 24 & 14 & 52 & 14.0 & 59 & 50.0 & 136 & 42.0\end{array}$ 242059 t. 6 G1 22.3 146 43.7

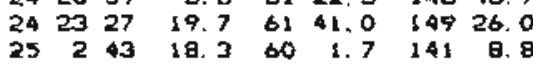

$\begin{array}{llllllll}25 & 7 & 77.8 & 60 & 17.1 & 141 & 13.3\end{array}$ $\begin{array}{llllllll}25 & 7 & 12 & 59.2 & 60 & 17.4 & 140 & 53.3 \\ 25 & 7 & 42 & 48.5 & 61 & 48.0 & 149 & 18.9\end{array}$ $\begin{array}{rrrrrrrr}25 & 1 & 1 & 27.1 & 62 & 14.5 & 149 & 37.1 \\ 29 & 11 & 6 & 54.7 & 60 & 10.6 & 141 & 38.3\end{array}$

$25 \quad 34 \quad 42 \quad 12.9$ bo $1.1 \quad 94037.8$ $\begin{array}{llllllll}25 & 14 & 57 & 12.2 & 61 & 5.3 & 149 & 47.8 \\ 25 & 15 & 39 & 48.3 & 54 & 56.8 & 140 & 45.6\end{array}$ $\begin{array}{llllllll}25 & \mathrm{t} 8 & 20.2 & \text { की } & 11.1 & 141 & 1.3\end{array}$ $251.6 \quad 6 \quad 4.0$ b1 $1.4 \quad 14953.5$ 45. $4.5 \quad 6 \quad 7 \quad 209 \quad 74 \quad 0.27$ 4.6 1. 14 b 144690.59 $\begin{array}{rrrrrrr}43.0 & 2.9 & 25 & 6 & 126 & 05 & 0.36 \\ 44.8 & 1.5 & 6 & 4 & 172 & 74 & 0.26\end{array}$ $\begin{array}{rrrrrrr}38.7 & 2.0 & 16 & 4 & 172 & 74 & 0.26 \\ & & & & 131 & 92 & 0.49\end{array}$

6. 21.1 \& 3 181 a5 0.05 $\begin{array}{lllllll}25.3 & 2.1 & 12 & 6 & 83 & 94 & 0.99\end{array}$ $\begin{array}{lllllll}3.5 & 1.2 & 6 & 2 & 173 & 110 & 0.20\end{array}$ $\begin{array}{rrrrrrr}9.8 & 1.7 & 13 & 6 & 112 & 49 & 0.17\end{array}$ 19.7 $1.6 \quad 9 \quad 5 \quad 150 \quad 500.20$ 2.7 $1.6 \quad 17 \quad 9 \quad 200 \quad 97 \quad 0.20$ $\begin{array}{lllllll}3.3 & 1.3 & 6 & 3 & 170 & 75 & 0.37\end{array}$

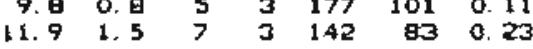

31. 日 2. 4 $\begin{array}{rrrr}7 & 120 & 99 & 0.44 \\ 2 & 230 & 118 & 0.12\end{array}$ $\begin{array}{lllllll}0.2 & 0 & 4 & 2 & 230 & 119 & 0.12 \\ 4 & 1.0 & 5 & 3 & 134 & 100 & 0.31\end{array}$

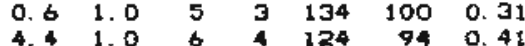
57. 21 25 1 2 147 80 0.37 $\begin{array}{lllllll}3.7 & 1.0 & 5 & 2 & 133 & 97 & 0.34\end{array}$ $\begin{array}{lllllll}3.1 & 0.7 & 4 & 2 & 139 & 79 & 0.34 \\ 4.4 & 1 & 4 & 2 & 154 & 101 & 0.20\end{array}$ $\begin{array}{lllllll}4.4 & 1.4 & 4 & 2 & 154 & 101 & 0.33\end{array}$ 29 1.4 19 日 190 be 0.97 $\begin{array}{llllll}5 & 0 & 318 & 165 & 1.12\end{array}$ $\begin{array}{lllllll}16.8 & 1.7 & 24 & 6 & 80 & 45 & 0.44\end{array}$ b. $\begin{array}{llllll}2.0 & 7 & 3 & 150 & 60 & 0.33 \\ 0.9 & 0.11\end{array}$ $\begin{array}{lllllll}9.5 & 1.3 & 8 & 3 & 117 & 76 & 0.07\end{array}$ $\begin{array}{llllllll}4.9 & 1 & 0 & 4 & 3 & 173 & 115 & 0.10\end{array}$ 38. $31.0 \quad 9 \quad 9 \quad 158 \quad 63 \quad 0.17$ $\begin{array}{rrrrrrr}44.7 & 2.4 & 20 & 16 & 201 & 104 & 0.54 \\ 2.1 & 1.3 & 9 & 4 & 117 & 45 & 0.37\end{array}$ $\begin{array}{rllllll}2.0 & 1.1 & 5 & 5 & 158 & 79 & 0.41 \\ 18.3 & 1.0 & 8 & 7 & 156 & 63 & 0.34\end{array}$ $\begin{array}{lllllll}18.3 & 1.0 & 8 & 7 & 156 & 69 & 0.34 \\ 11.9 & 1.4 & 6 & 5 & 185 & 63 & 0.30\end{array}$ 4 is $\begin{array}{llll}146 & 01 & 0.29\end{array}$
ERH EFI O AZI DIPI SEI AZZ DIE2 SEZ AZ3 BIPS SES

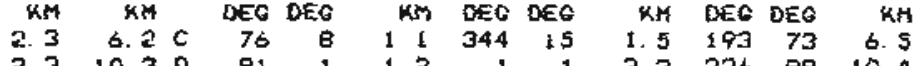
$\begin{array}{rrrrrrrrrrr}2.3 & 10.30 & 91 & 1 & 1.2 & 34 & 15 & 1.5 & 193 & 73 & 6.5 \\ 1.6 & 2.7 & 319 & 1 & 0.6 & 49 & 25 & 1.3 & 226 & 99 & 10.4 \\ & & & & 1.1 & 227 & 65 & 2.9\end{array}$

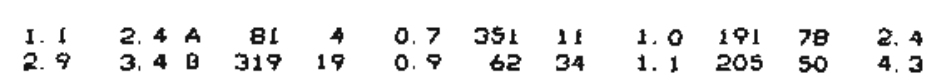
$\begin{array}{rrrrr}2.0 & 1.9 & \text { A } & 268 & 6 \\ 1.9 & 1.1 & \text { A } & 17 & 6 \\ 2.7 & 6.7 & \text { C } & 87 & 5 \\ 1.9 & 2.2 & \text { A } & 16 & 3\end{array}$

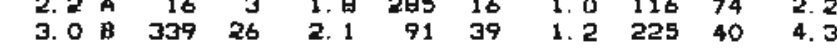

$\begin{array}{lllllllllll}3.5 & 5.9 \mathrm{C} & 303 & 0 & 0.9 & 33 & 26 & 2.3 & 213 & 64 & 6.4\end{array}$

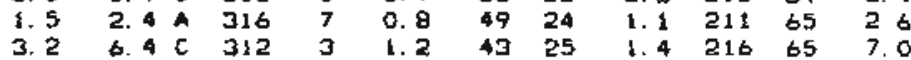

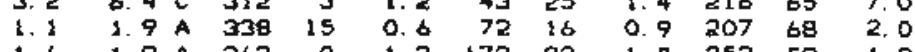

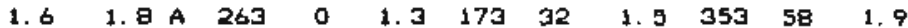

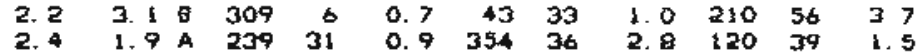

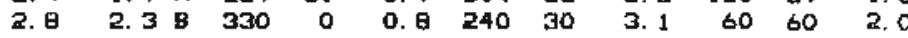

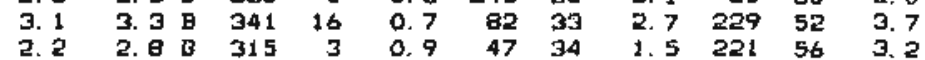
75. 0 3. 1 . 15

1. 9 A $79 \quad 29$

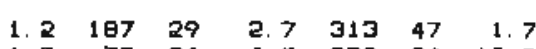

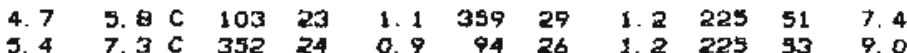

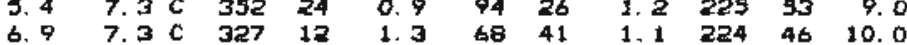

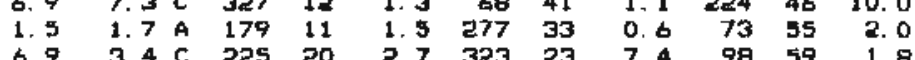

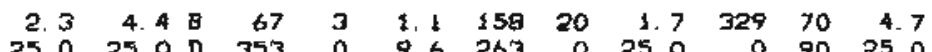

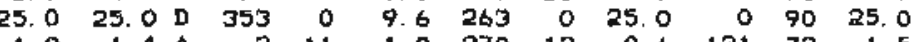

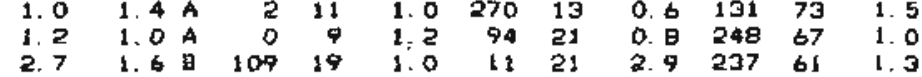

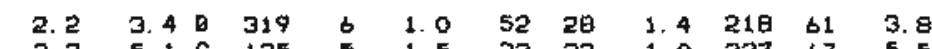

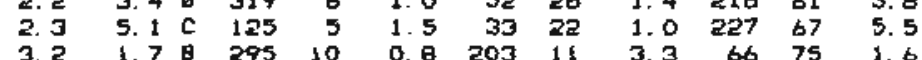

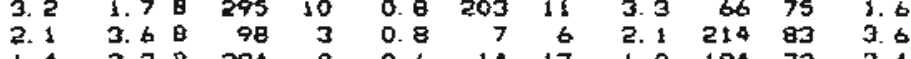
$\begin{array}{rrrrrrrrrrrr}3.3 & 3.3 & B & 109 & 11 & 0.5 & 5 & 44 & 3.0 & 210 & 44 & 3.6 \\ 1.6 & 2.4 & \text { A } & 300 & 18 & 1.2 & 203 & 22 & 0.7 & 66 & 61 & 2.7 \\ 4.4 & 2.6 & \text { B } & 207 & 1 & 4.4 & 116 & 15 & 0.9 & 301 & 75 & 2.7\end{array}$

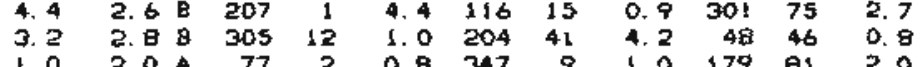




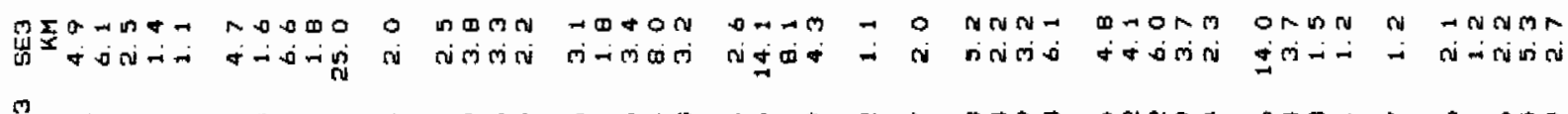

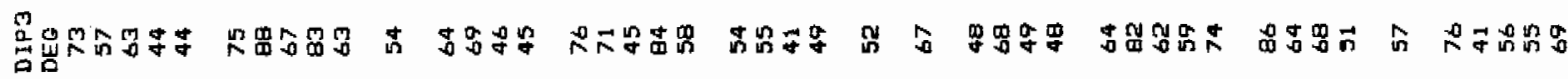

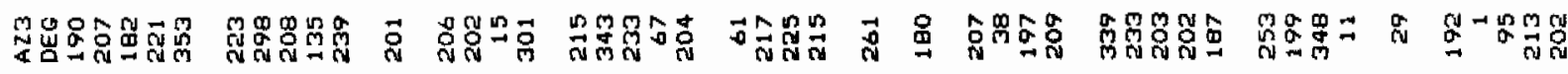

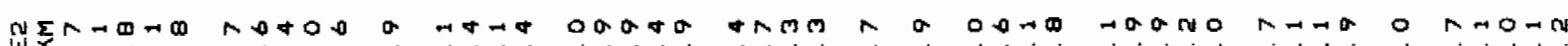

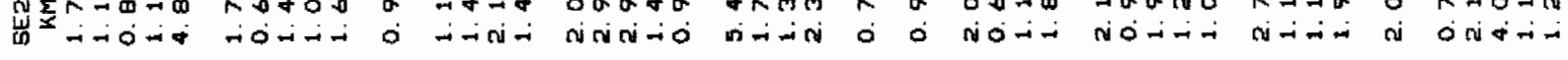

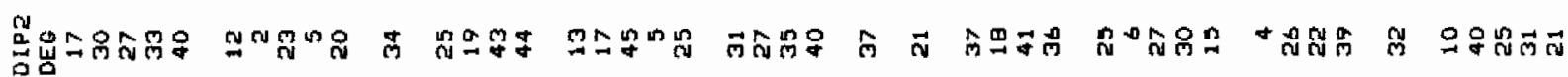

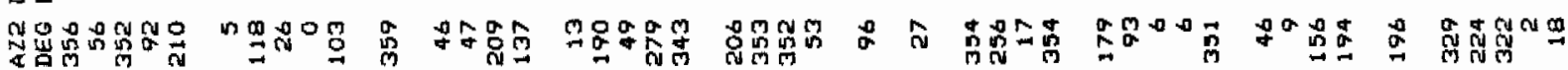

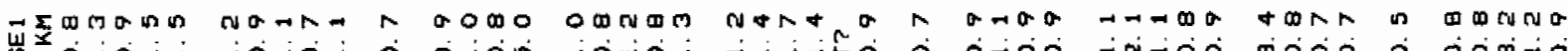

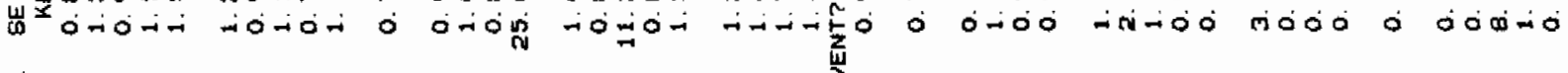

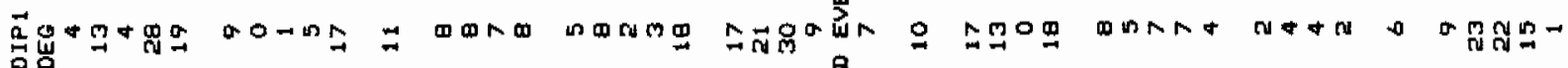

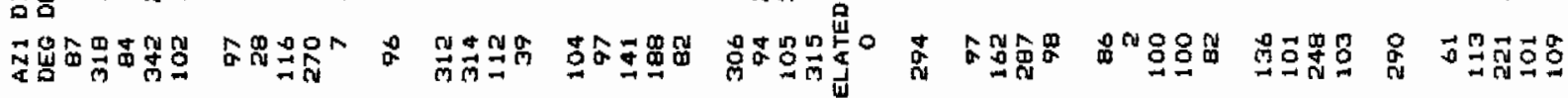
O NEMNMN 0 岳

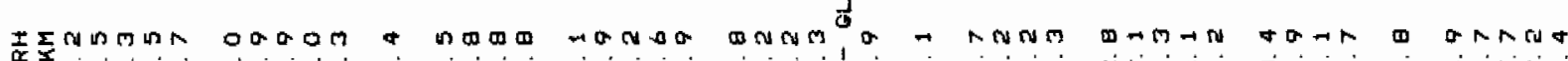

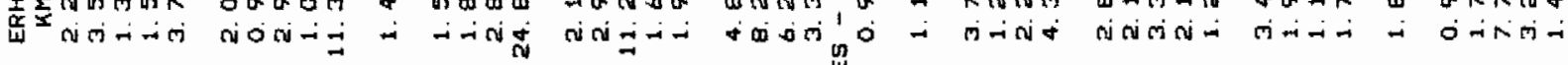

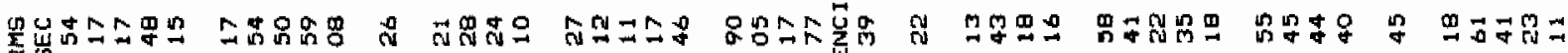
品

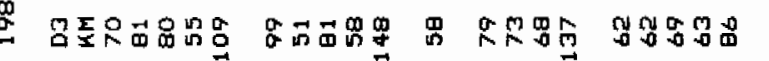

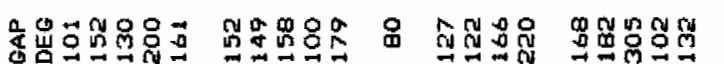

o

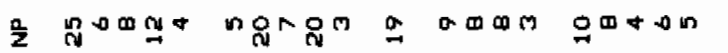

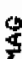

ONANO OMNOD

$\ln _{\rightarrow \infty} \rightarrow 000$

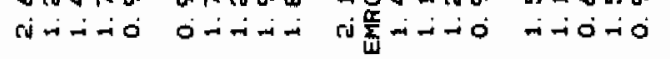

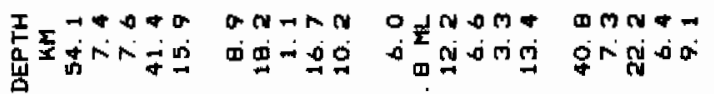
ri

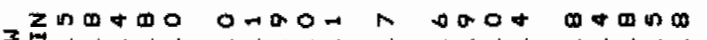

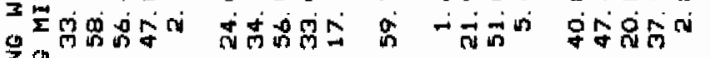

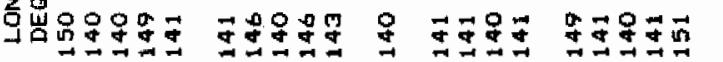

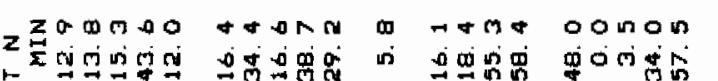

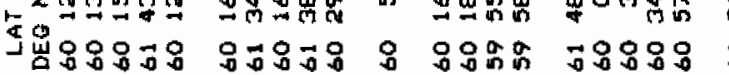

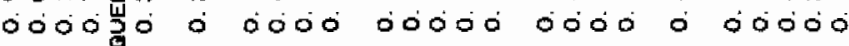

munono

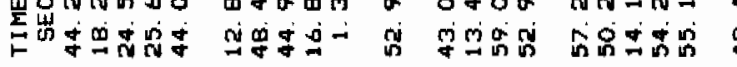

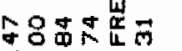

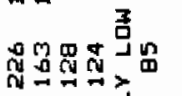

$\operatorname{Rog}$

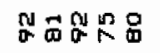

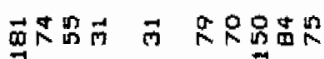

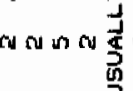

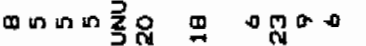

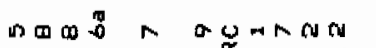

-

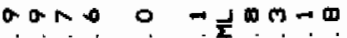

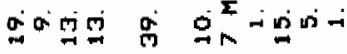

ai

otmo a o v o

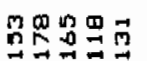

$\operatorname{mag}$

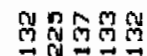

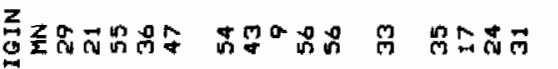

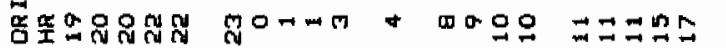

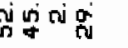

की

$\infty \rightarrow \infty 0$

Noom a 000 in

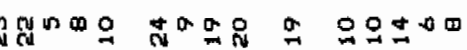

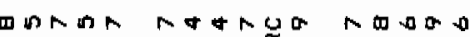

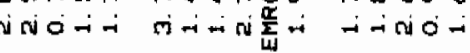

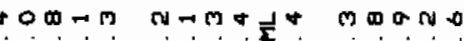

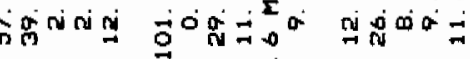

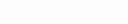
ri

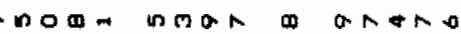

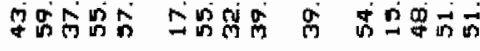

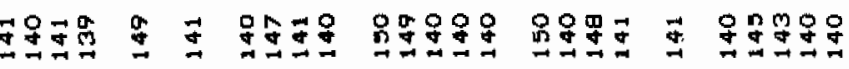

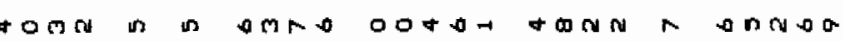
ลำ

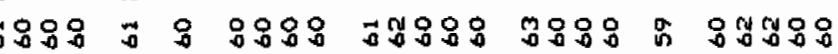

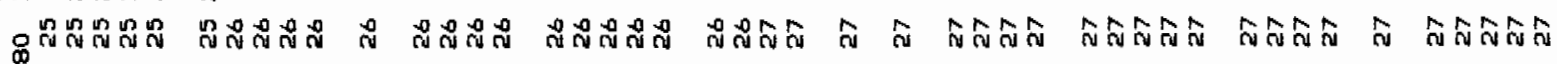
品 
SDUTHERN ALASKA EARTHQUAKEg, JAN - MAR 1980

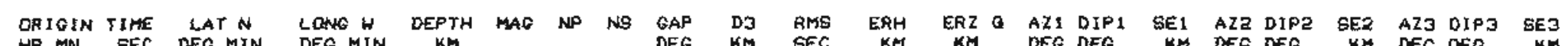
1990 HF MN SEC DEOMIN DEO MIN KM KN

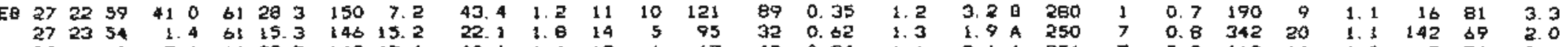

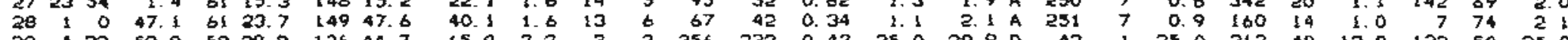

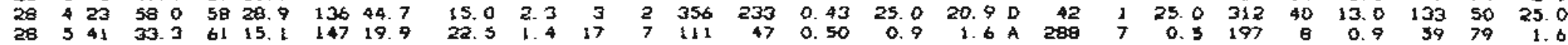

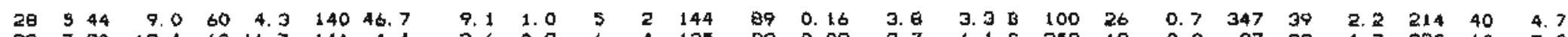

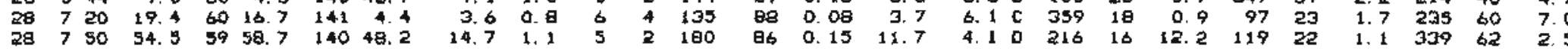

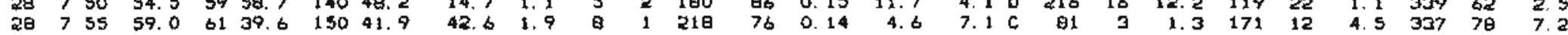

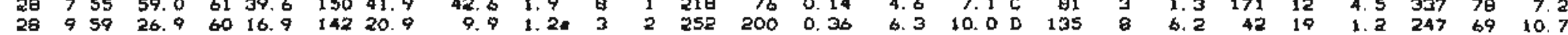

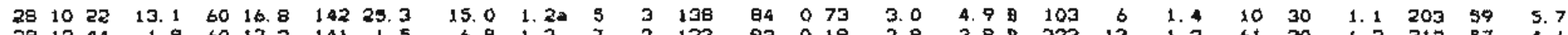

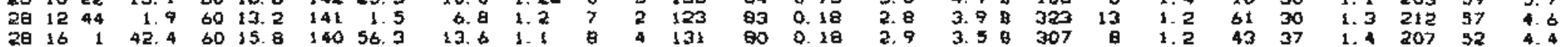

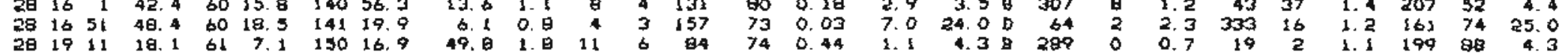

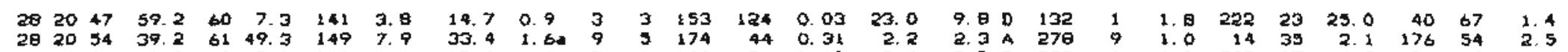

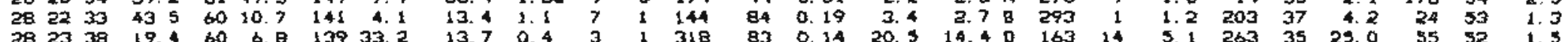

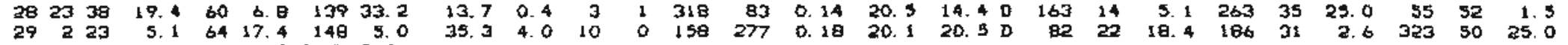

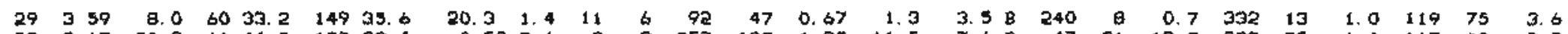

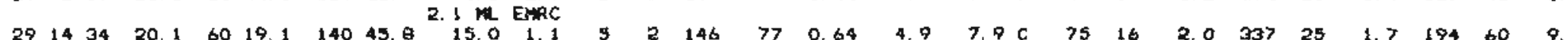

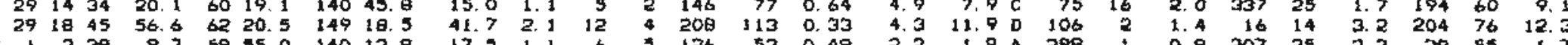

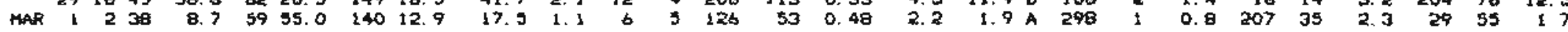

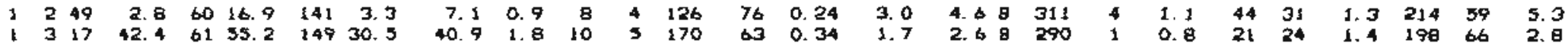

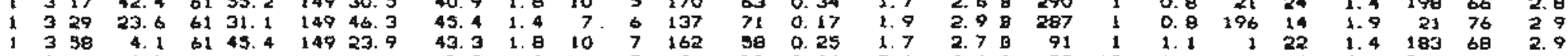

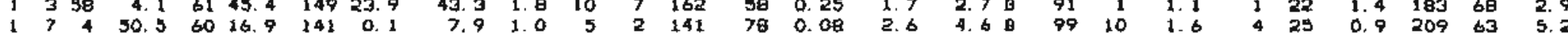

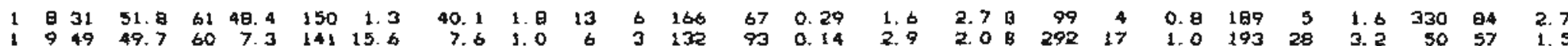

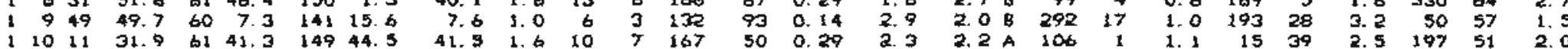

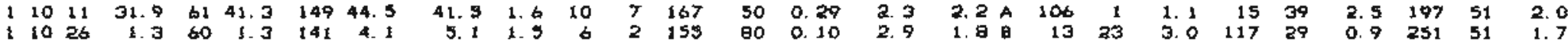

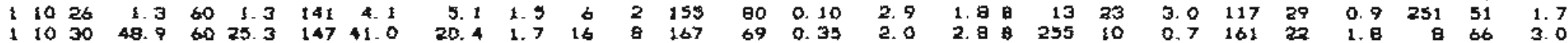

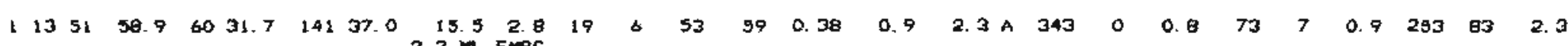

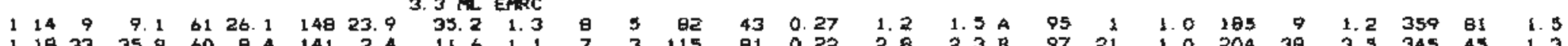

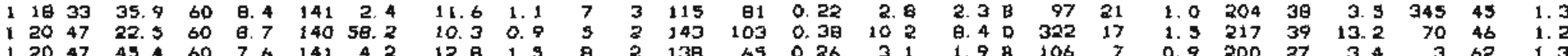

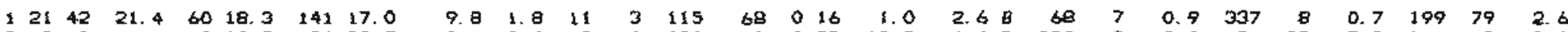

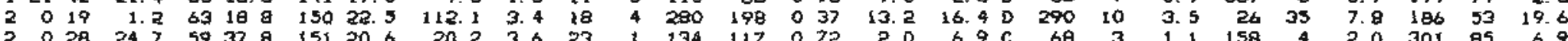

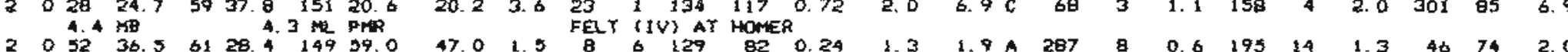

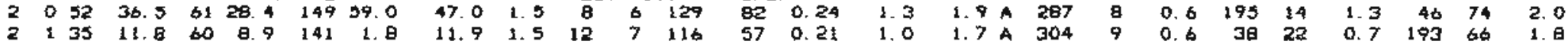


留

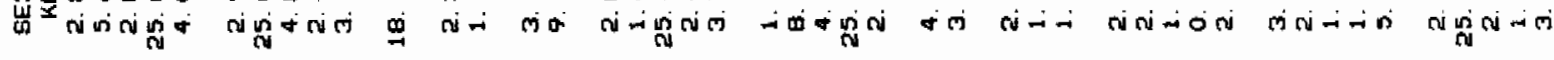

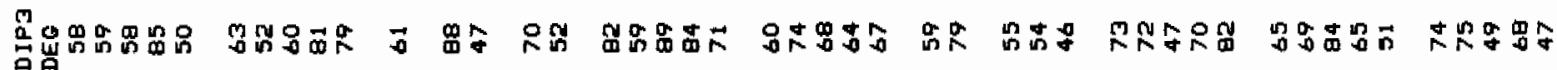

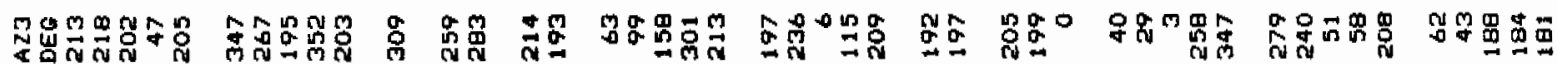

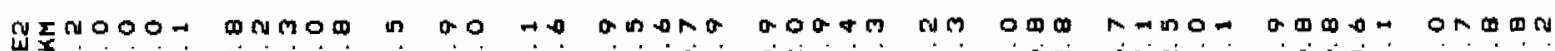

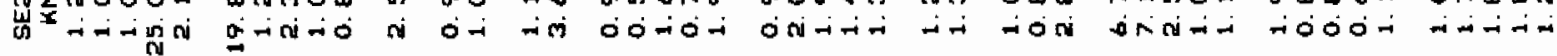

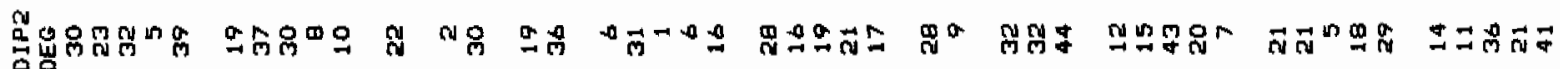

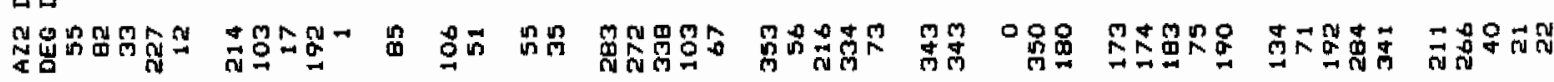

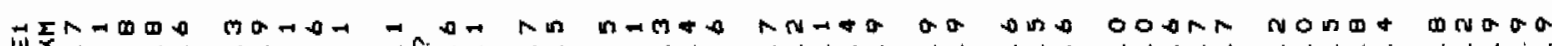
出

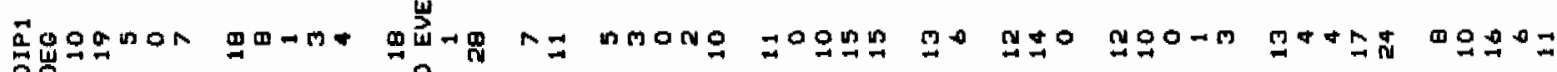

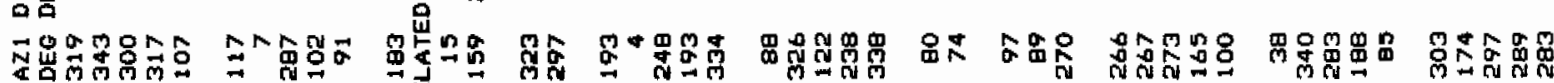

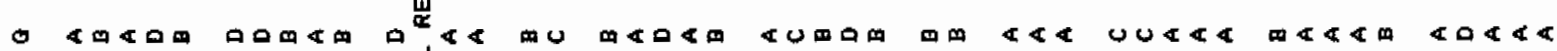

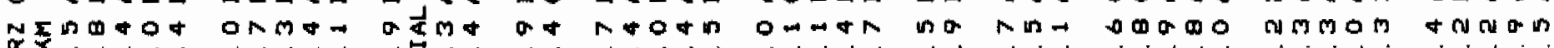

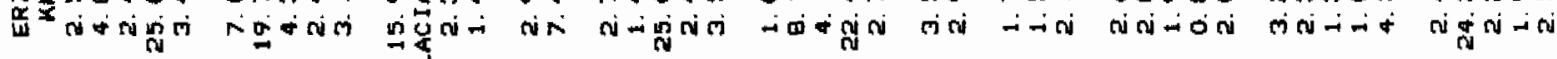

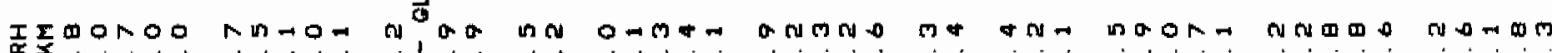

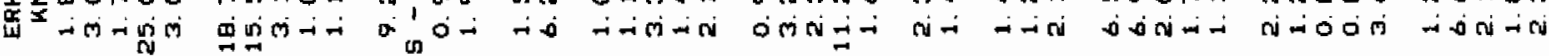

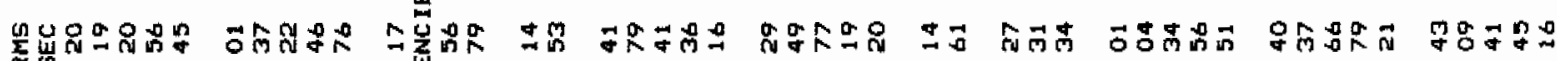

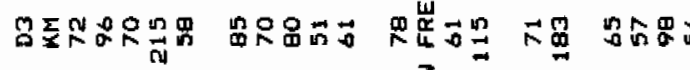

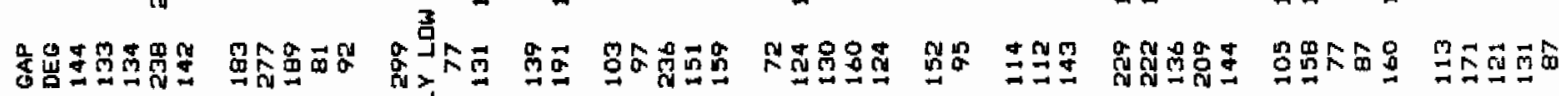
$\frac{6}{2} 0-00 m$ 문

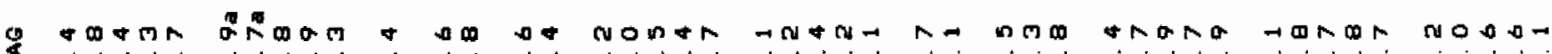
$\frac{4}{2}$ -

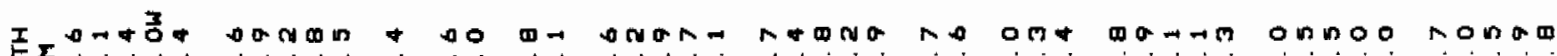

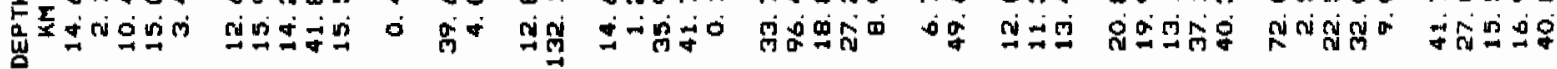

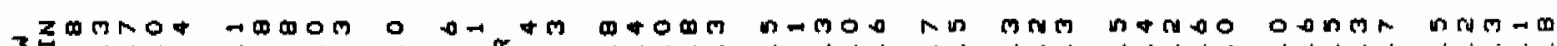

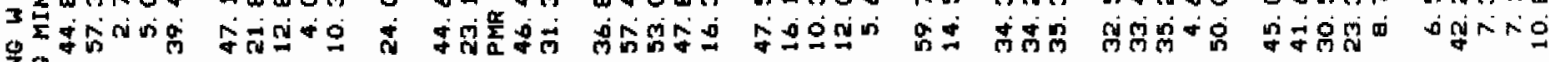

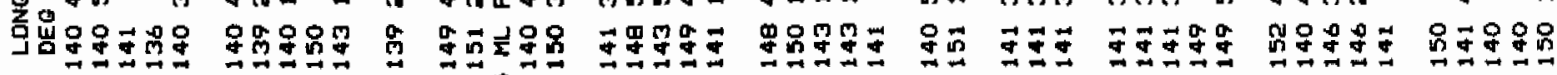

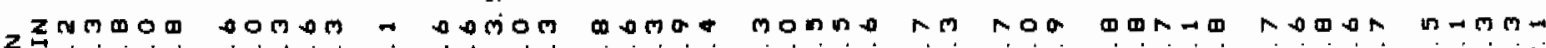

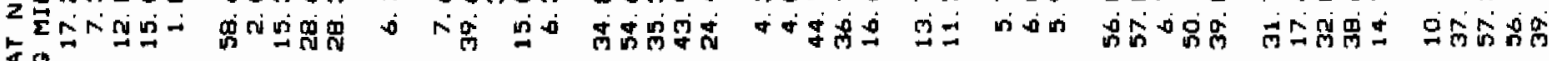

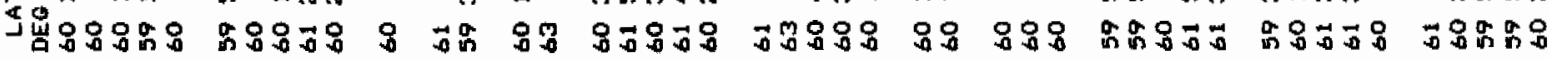

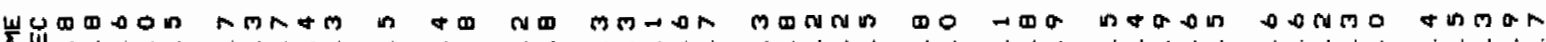

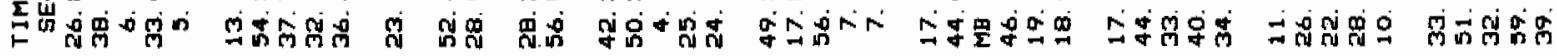

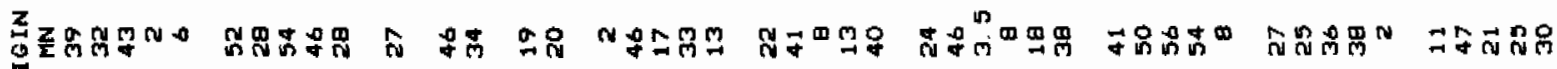

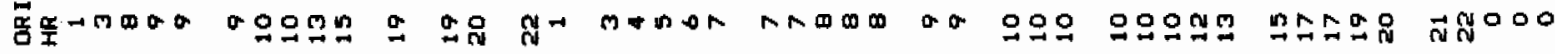

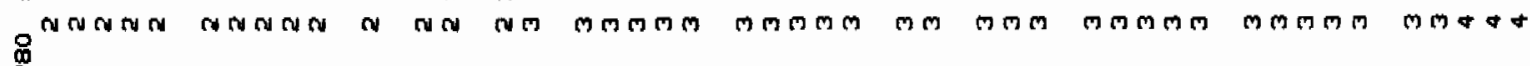
$\stackrel{\substack{0 \\ 0}}{\frac{\pi}{2}}$ 
ORIGIN TIHE LATN LOHEW DEPTH MAG NW NG QAP DJ RMG 1960 HAR MN SEC DEG MIN DEQ MIN KM

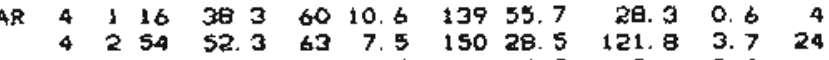

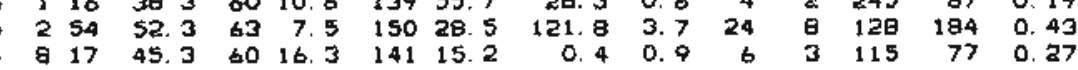

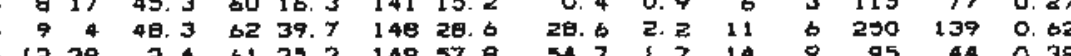

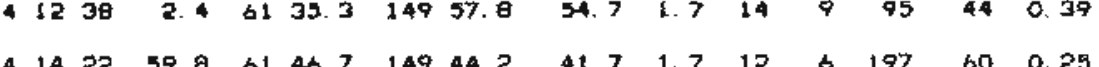

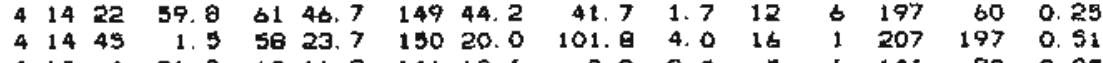
$415 \div 21.0$ to 11.214110 .00 .90 .5951141900 .09

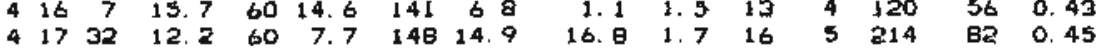

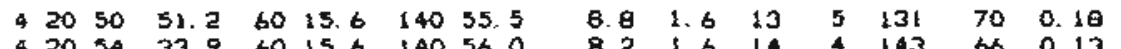

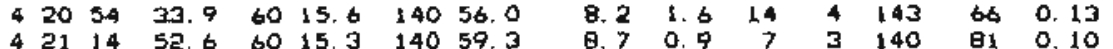

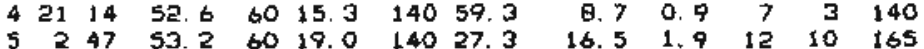

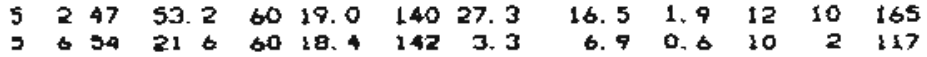

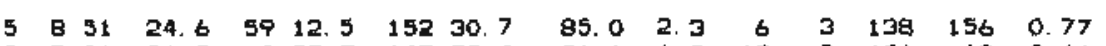

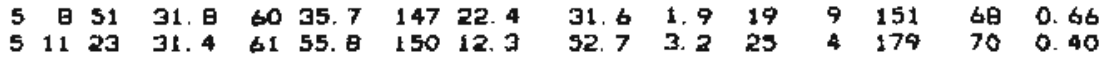
5125141.1 61 $24.0 \quad 149 \quad 8.2$ 28. 9 0.9 10 b 99 36 045

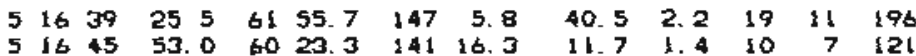
91711 19.

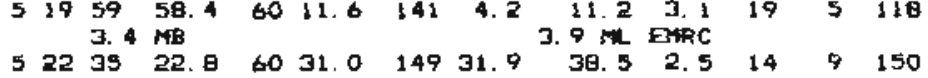

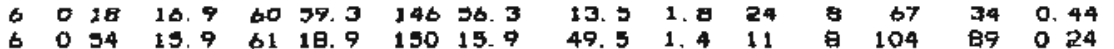
$\begin{array}{lllllllllllll}6 & 2 & 17 & 2.0 & 60 & 11.3 & 141 & 10.3 & 3.9 & 1.7 & 16 & 6 & 113\end{array}$

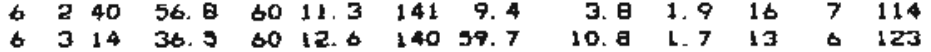

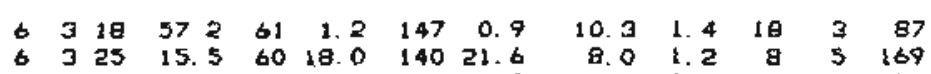

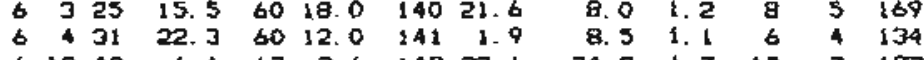

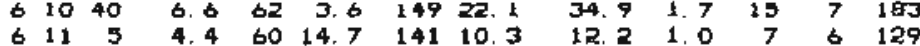

6. 412254.3 60 $5.3 \quad 141 \quad 15.9$ $\begin{array}{lllllll}6.11 & 32 & \text { 日. } 1 & 60 & 28.0 & 148 & 16.3\end{array}$

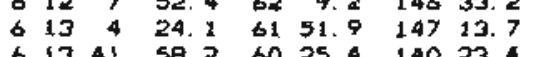

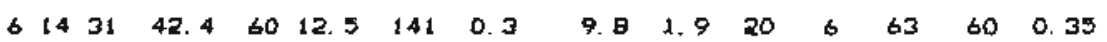

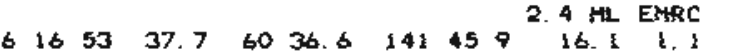

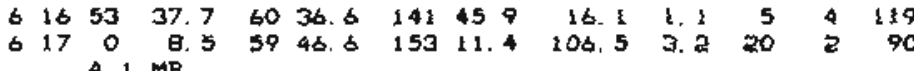
b. 2017 31.6 $6014.5 \quad 14046.1 \quad 11.0 \quad 1.0 \quad 5 \quad 3140$

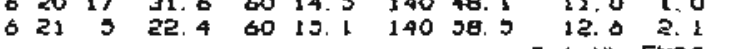
910.50

$\begin{array}{ll}79 & 0.73 \\ 64 & 0.73\end{array}$ 640.36 $116 \quad 0.41$

5. $51.0 \Rightarrow \$ 120$ $97 \quad 037$

$\begin{array}{lllllll}12.5 & 1.5 & 16 & 2 & 182 & 60 & 0.48\end{array}$

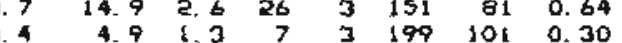
os 0.15 $\begin{array}{rrrr}2.4 & 20.0 & 0 & 253 \\ 2.4 & 4.1 & 8 & 320\end{array}$

ERH ERZ G AZI DIPI SE1 AZE DIPZ SEZ AZJ DIP3 BE3 $7.72 .1 C$ DEG DEQ

3. $12.1 \mathrm{D} 137$

(a) 241 2. $\begin{array}{lll}315 \\ \text { 2. }\end{array}$

2. 0 2. 5 A 110 3.6 6. 3 C $320 \quad 13$ $\begin{array}{lllll}0.9 & 2.3 & \text { A } & 315 & 10 \\ \text { 1. } 9 & 2.7 & \text { B } & 245 & 18\end{array}$

$\begin{array}{llll}3 & 2.5 & 300 \\ 1.3 & 2 & \text { A } & 327\end{array}$

$\begin{array}{rrrrr}\text { 2. } 3 & 3.7 & \text { B } & 327 & \text { B } \\ 1.9 & 2.4 & 20\end{array}$ $\begin{array}{lllll}1.0 & 2.1 & \text { A } & 311 & 1 \\ 2.2 & 3.7 & 6 & 349 & 12\end{array}$ 4. $5 \quad 11.2 \mathrm{D} 214$ $\begin{array}{rrrr}1.1 & 3.5 \mathrm{~A} & \mathrm{BS} & 4 \\ 2.2 & 0\end{array}$ $\begin{array}{rrrrrr}1.1 & 1 & 7 & A & 113 & 7 \\ \text { 2. } 5 & 1.5 & E & 199 & 18\end{array}$

2. 23.29100 1. 1 3.0. 341 3

2. 2 1. 8 A $93 \quad 28$ KM DEO OES 日. $0 \quad 119 \quad 12 \quad 1.3 \quad 28077$ $\begin{array}{lllllll}3.6 & 47 & 7 & 3 & 243 & 83 & 12.2\end{array}$

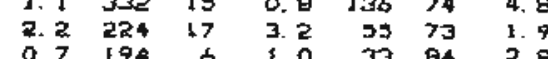
$\begin{array}{lllllll}1.0 & 200 & 11 & 2.0 & 15 & 79 & 25\end{array}$ $\begin{array}{lllllll}7.1 & 35 & 10 & 1.9 & 203 & 60 & 17.6\end{array}$ $1.1 \quad 26 \quad 24 \quad 1.6 \quad 204$ a 7.1 $\begin{array}{rrrrrrr}0.6 & 47 & 11 & 0.7 & 164 & 75 & 2.3 \\ 0.0 & 148 & 18 & 1.7 & 17 & 64 & 3.0\end{array}$ $\begin{array}{lllllll}0.8 & 31 & 16 & 1.1 & 193 & 73 & 2.6\end{array}$ $\begin{array}{lllllll}1.0 & 60 & 19 & 1.0 & 215 & 69 & 2 .\end{array}$ $\begin{array}{lllllll}0.6 & 41 & 10 & 1.0 & 215 & 60 & 2.1 \\ 0.7 & B 5 & 25 & 1.2 & 236 & 62 & 4.2\end{array}$

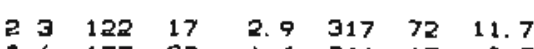
$\begin{array}{lllllll}0.6 & 177 & 23 & 1.4 & 346 & 67 & 0.9\end{array}$

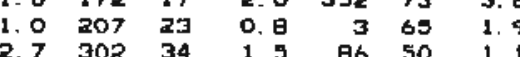
$\begin{array}{lllllll}0.7 & 19 ! & 21 & 2.0 & 0 & 67 & 3.3\end{array}$ $\begin{array}{lllllll}0.8 & 59 & 9 & 1.0 & 239 & 82 & 3.0\end{array}$ $\begin{array}{lllllll}0.9 & 72 & 6 & 1.0 & 225 & 63 & 3.0 \\ 0.7 & 39 & 29 & 0.9 & 201 & 60 & 2.2\end{array}$ $\begin{array}{llllllll}0.9 & 195 & 35 & 2.5 & 324 & 42 & \text { 1. } 5\end{array}$

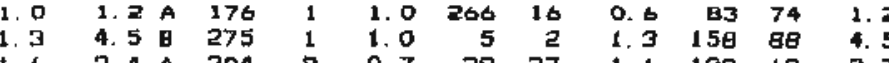

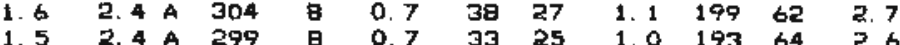

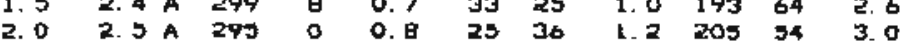

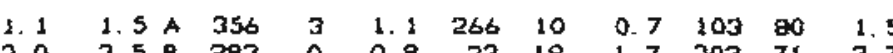

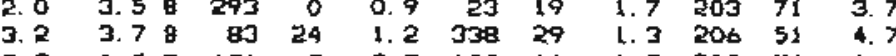

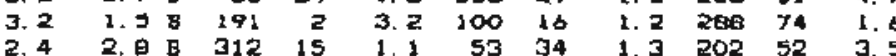

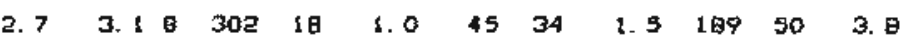

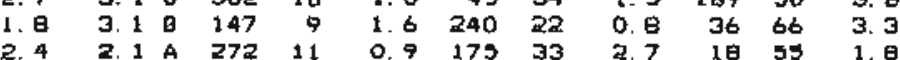

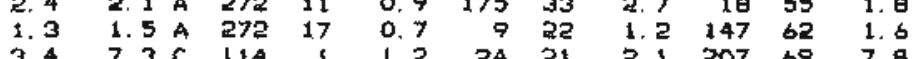

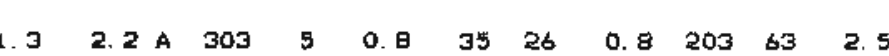
2. 6 ML EMRC 


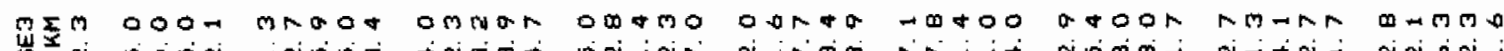

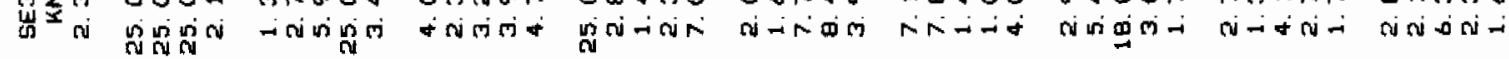

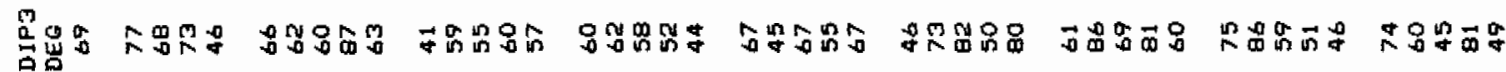

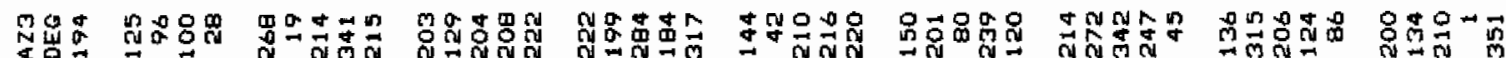

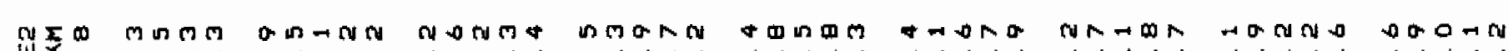

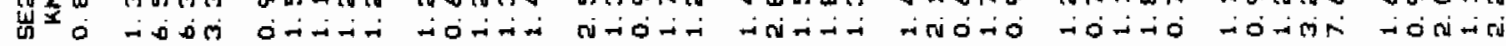
荌若

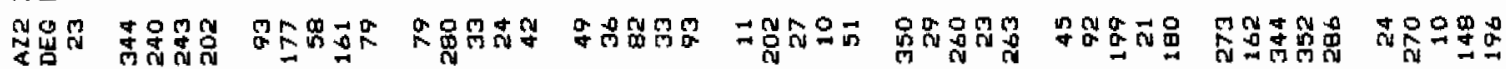

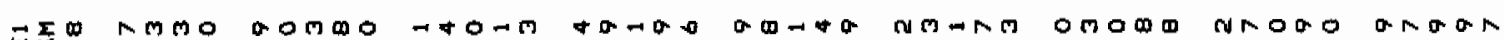
㟧至

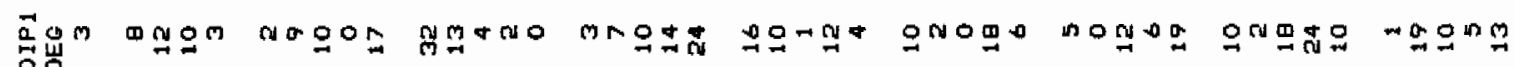

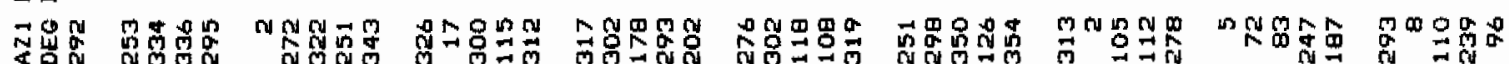
0 व

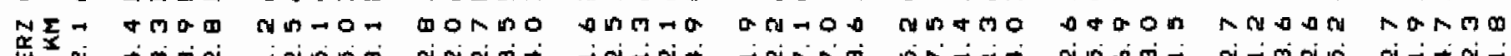

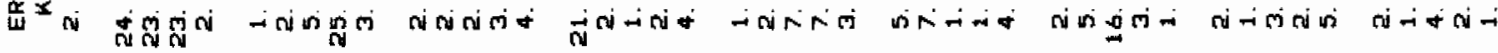
I焉

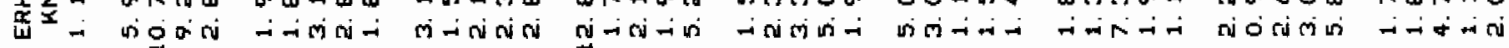

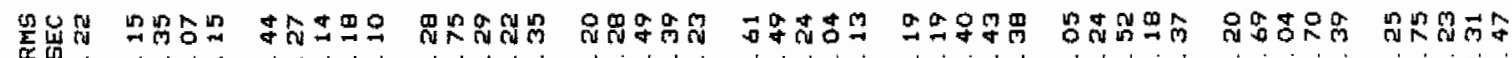

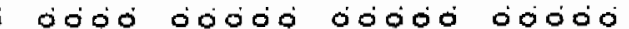
ióóo íóó

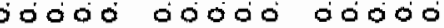

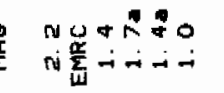

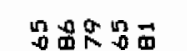

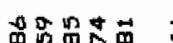

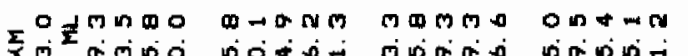

पु

$\rightarrow$ in

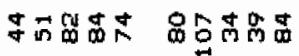

政

$\sin 0 \sin$

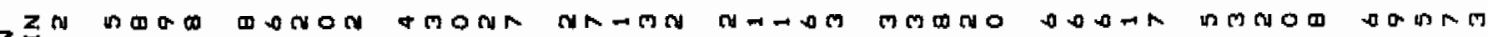

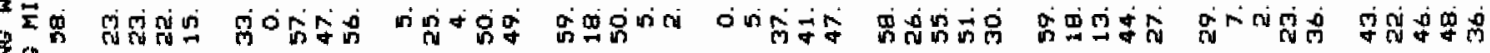

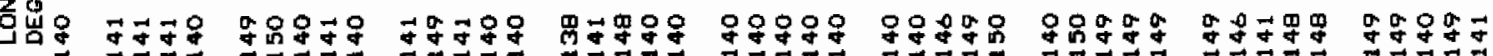

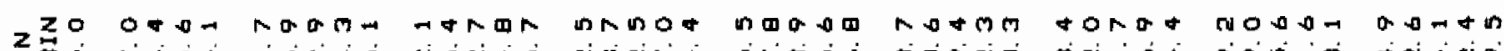

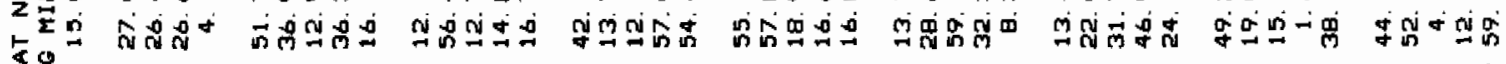

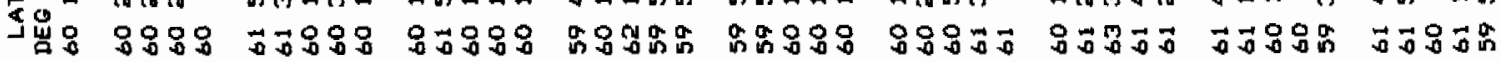

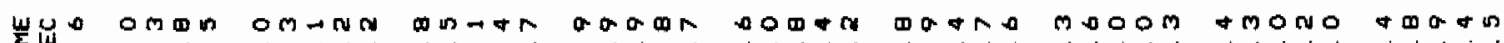

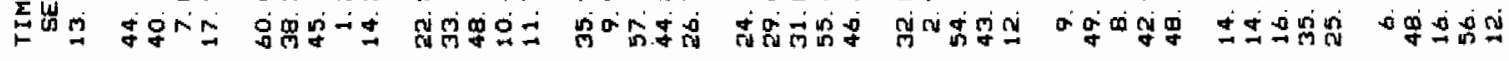

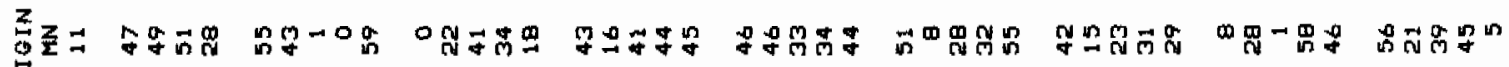

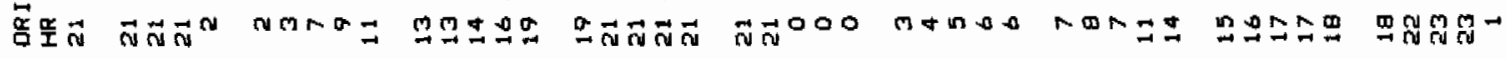

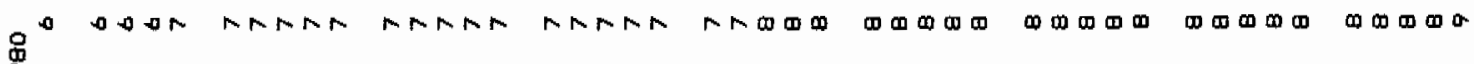




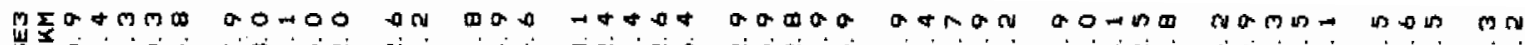
岁

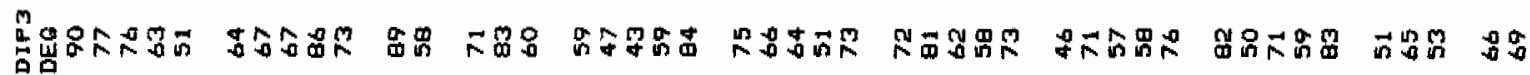

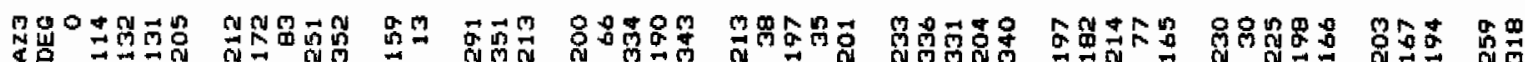

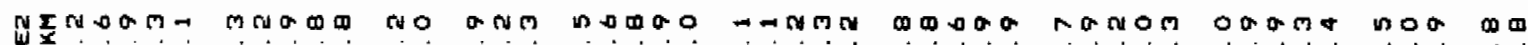

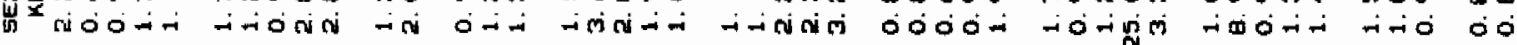

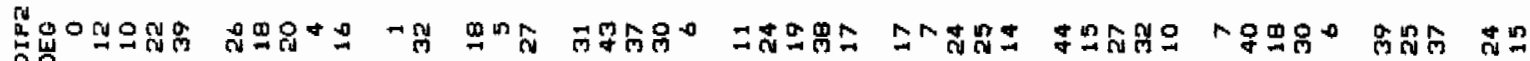

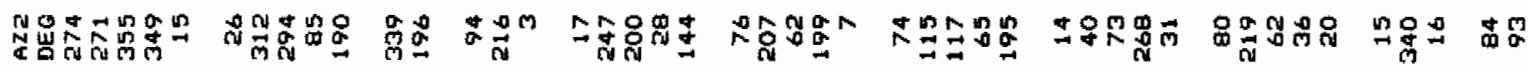

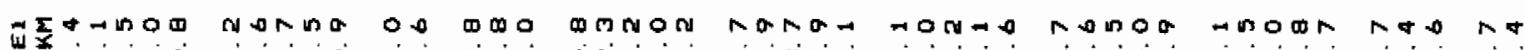

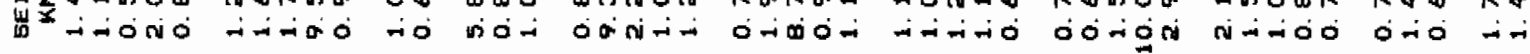

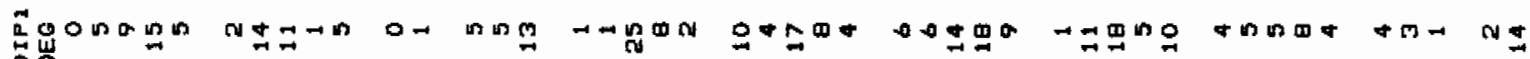
吕

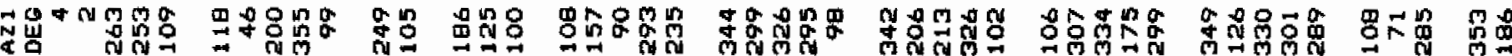

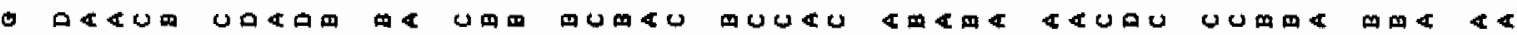

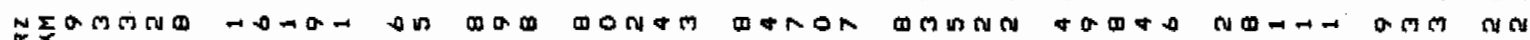

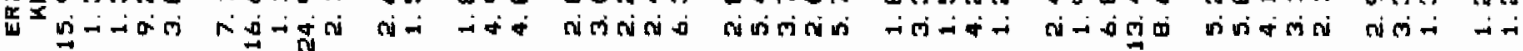

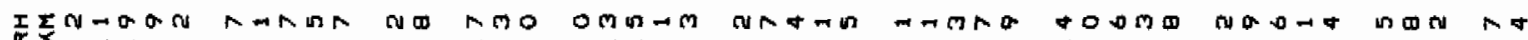
安

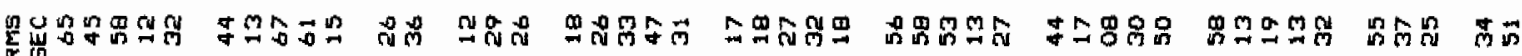

óó óóó ióó

ióoi ióoió ஸீं0்

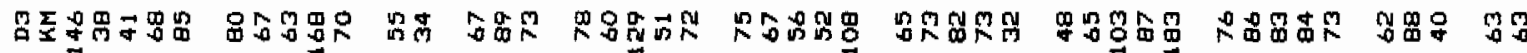

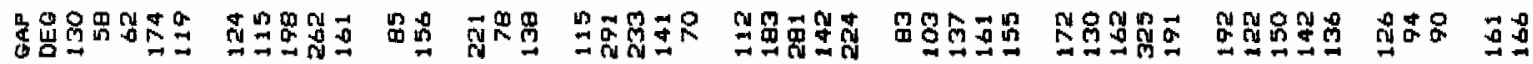

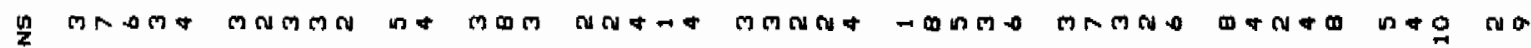
द

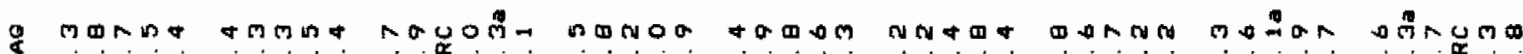

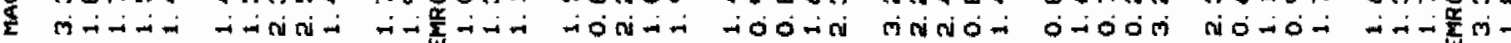

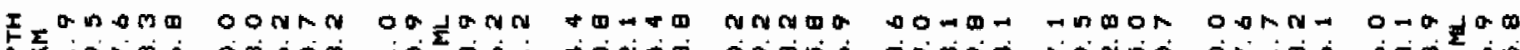

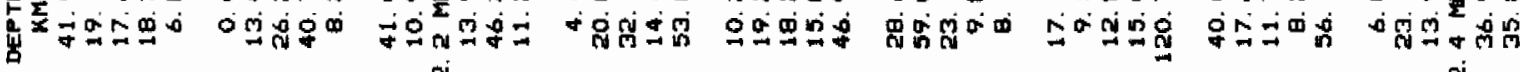
ni

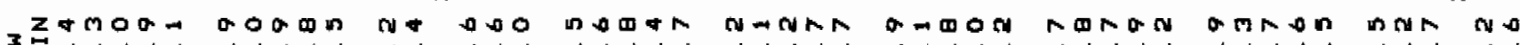

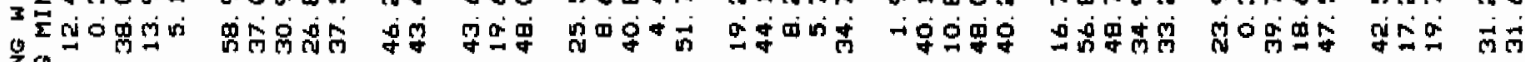

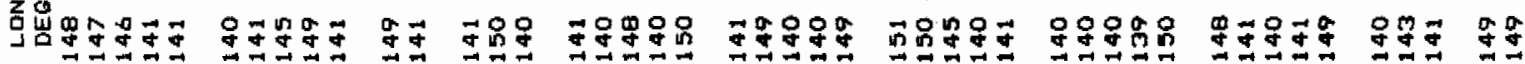
z zon

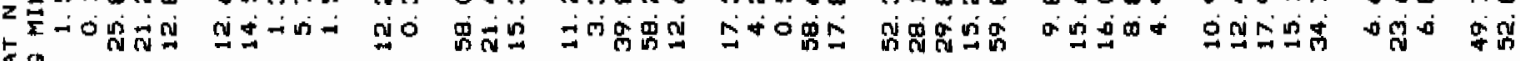

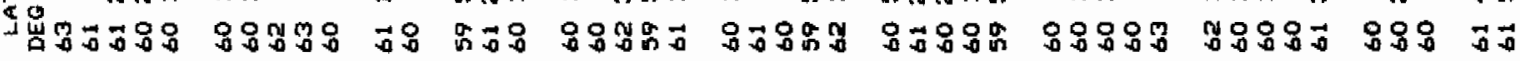

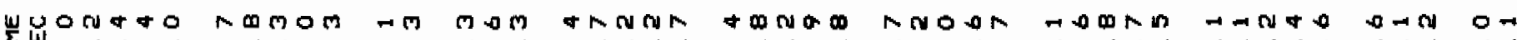

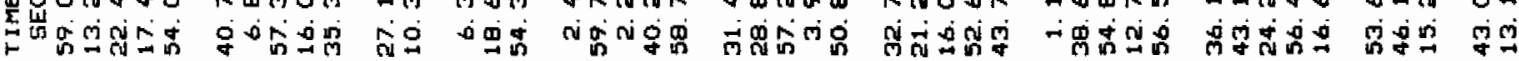

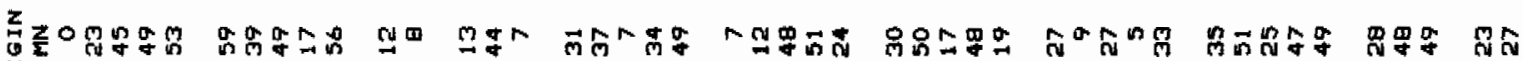

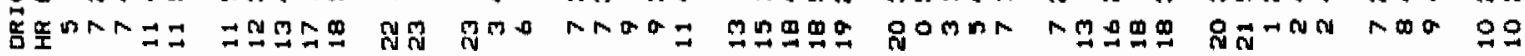

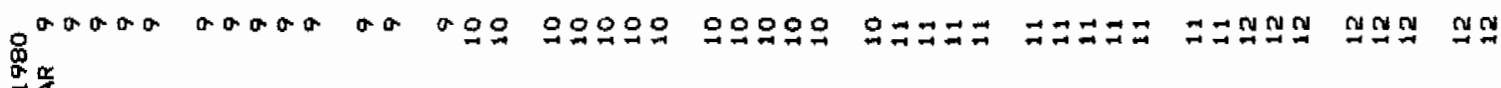
$\frac{0}{\frac{\alpha}{2}}$ 


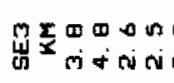

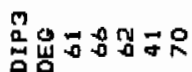

ำมำลำำกำ

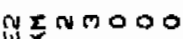

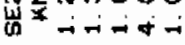

ํํำ

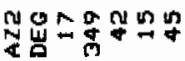

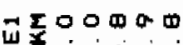

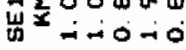

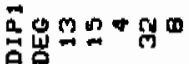

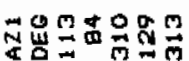

0 回的公东

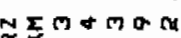
击

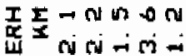

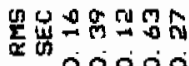

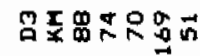

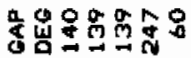

on on

$\frac{9}{2} \quad 0 \times 0 \stackrel{0}{\rightarrow}$

a $\quad 00 \mathrm{Or}-0$

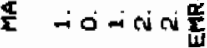

I $m$ 助

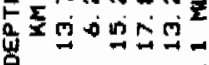

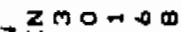

3 E

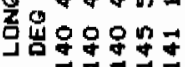

z $z$ काष十

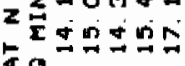

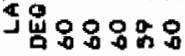

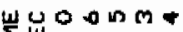

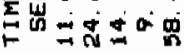

否至的电的品员

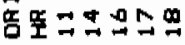

尊哭 N

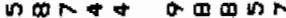

- ता

ind 4

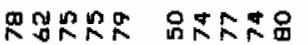

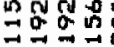

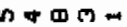

$0-0 \dot{0}$

$\Rightarrow$ 思出

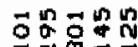

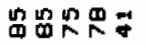

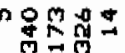

$\rightarrow$ ता 0

$\cos 0=$

t $\mathrm{di}$ imo

कंष्म

$\ln =$

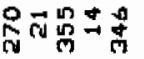

由 O m

oं

Mnto

- ते तi

nog o O ONA

$\rightarrow 0 \mathrm{x}$

$\rightarrow \underset{\alpha}{2} \frac{0}{2}$

m m

$+40=$

- तं मिं

DOmOd

onkar

ondi

क

ठे तें

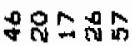

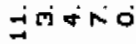

óocóo

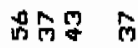

diojoo

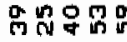

o0000

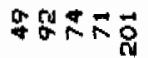

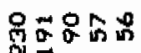

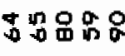

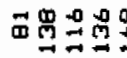

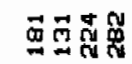

o. तू त क क

$\rightarrow$ m n $\rightarrow$

momog 음

nn n

- $\rightarrow$ m n

-ió

लें

an m o

a $=00$

momn

ㅁำ

m 4 - 1

$0000=$

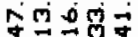

Aक्षण

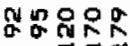

ต $\mathrm{N} \infty$

nल०

ल तथ 0 n

i - i $\mathrm{N}$

- $\mathbf{m}$ N⿴囗十

in

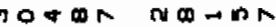
$\operatorname{xing} \sin$ का

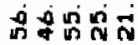

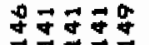

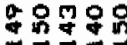

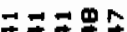

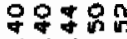

- moon mamo

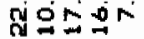

क्षेंक्ष

由 m क 由

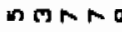

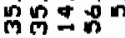

ำ

웅요

का

구응

80950

89050

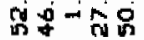

Drin तो

$\infty+00-$

a m Non

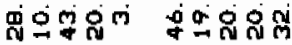

$\ddot{2} 00+0$

gan in 0

ำกำ

东哭识范

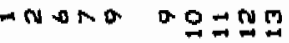

品然品最。

드ำ तู

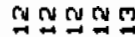

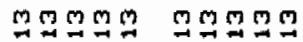

$\stackrel{m}{9} \underset{7}{9}$
$0 \rightarrow-N=0$

$+\log x=0$

no mo

$0 m 0$

ल ताँ

$\rightarrow 0 \mathrm{~d}$

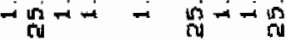

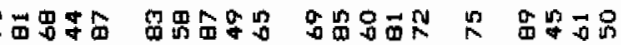

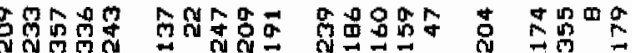

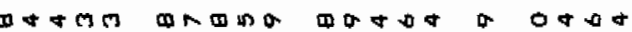

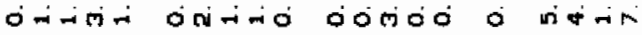

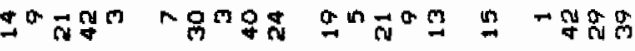

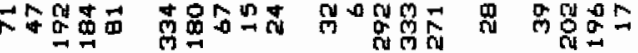

NOMON

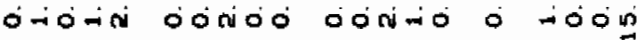

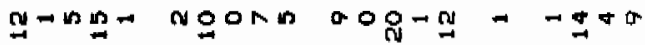

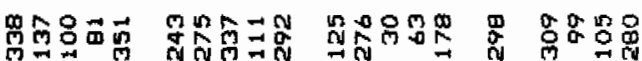

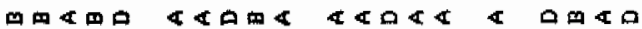

MOO+U +

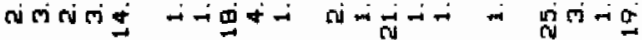

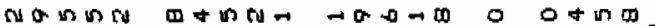
- - ना

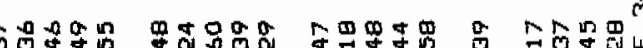

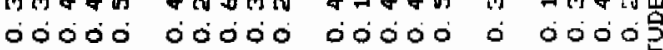

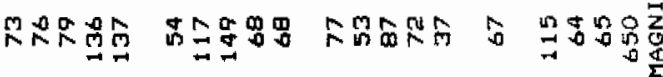

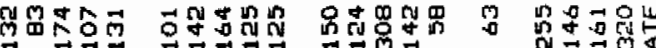

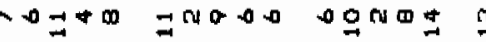

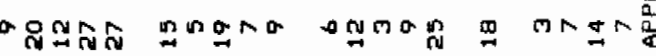

onoto and

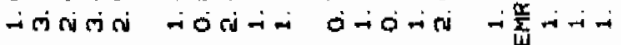

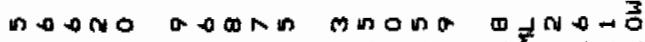

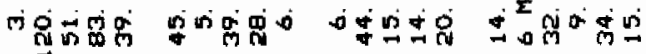

ri

mom mamom noOnm

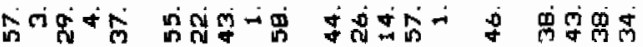

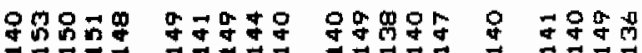

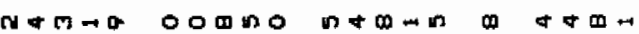

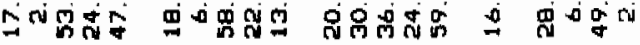

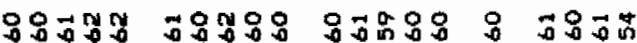

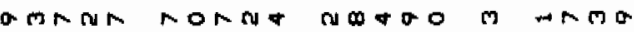

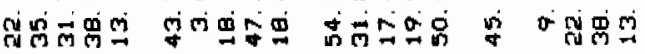

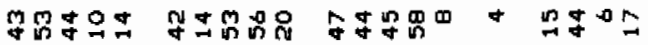

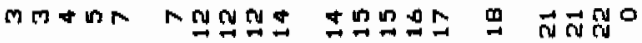

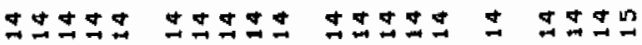


ORIGIN TIME LAT N LDNO $W$ DEPTH MAG NF NE GAP DJ RTM 1980 HF WN SEC DEG MIN DEQ MIN KM . DE KM SEC

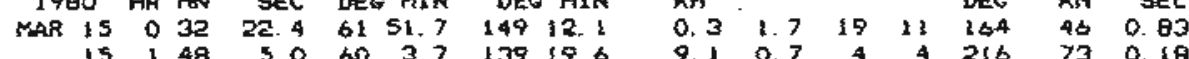
15148 5. 15 60 $3.7 \quad 13919.6$

$15323 \quad 6.5 \quad 6010.9 \quad 14050.7$ $\begin{array}{lllllll}15 & 52 & 27.3 & \text { bo } & 5.9 & 140 & 43.2 \\ 15 & 10 & 51.3 & \text { 61 } & 25.8 & 146 & 37.5\end{array}$

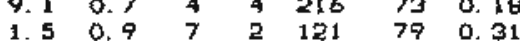
$\begin{array}{llllllll}15 & 11 & 7 & 41.7 & 61 & 49.3 & 149 & 28.6\end{array}$ $\begin{array}{rrrrrrrr}15 & 11 & 55 & 7.6 & 61 & 2.7 & 146 & 72.9 \\ 15 & 13 & 55 & 49.9 & 60 & 3.7 & 237 & 21 .\end{array}$ $151433 \quad 13.4$ 61 $49.0 \quad 149$ 28. 5 $151437 \quad 48.062 \quad 121497.9$

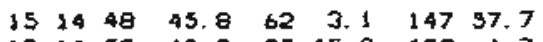
$151459 \quad 40.5 \quad 5945.6136 \quad 1.7$ $1517 \quad 16$ 52. 3 59 $50.0 \quad 141 \quad 36.4$ 131932 27.5 6129.1 149 51.0

$151956 \quad 23.2$ 60 15.6 140 55. 152029 26. 3 bo e.b 14111.9 $152057 \quad 25$ of $2.6 \quad 146 \quad 320$

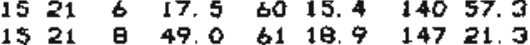
$\begin{array}{lllllll}1522 & 37 & 41.2 & 60 & 16.9 & 140 & 44.7\end{array}$ $\begin{array}{rrrrrrr}4.0 & 0.9 & 5 & 3 & 145 & 90 & 0.22 \\ 21 . & 2.6 & 27 & 4 & 62 & 41 & 0.54\end{array}$ $4,21,6$ b. 6 2. 2.4 3 206 73 o. 2.2 0. $12 \quad 5 \quad 171$ 60 0.8

$34.7 \quad 3.0 \quad 18$ $\begin{array}{llll}3 & 1.2 & 3 & 311\end{array}$ 19.9 1.4 8 a 519

$\begin{array}{ll}00 & 0.20 \\ 92 & 0.25\end{array}$ $\begin{array}{ll}73 & 0.21 \\ 65 & 0.25\end{array}$ $\begin{array}{ll}65 & 0.25 \\ 40 & 0.50\end{array}$ 12. $51.510 \quad 4 \begin{array}{llll}131 & 70 & 0.13\end{array}$ 7.2 2. 2 29 9 5150 114 0.12 8.6 1.2 (1)

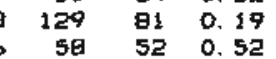
ERH ERZ $O$ AII DIPI BEL AZZ DIPZ SER AZ3 DIP3 SE3 KM NM DEG DEQ HM DEO DEC KM DEG DEG $3.5 \quad 1.5 \mathrm{~A}$ 259 16 3. 5. $\begin{array}{rrrrr}5.8 & \text { b. } 4 \text { C } & 104 & 13 \\ 0.7 & 1.4 \mathrm{~A} & 11 & 3\end{array}$ 0.6 J $355 \quad 20 \quad 1.3 \quad 133$ b4 1.4 15 28 l. 2030 54 5.7 1. $1340 \quad 22 \quad 1.4$ 213 60 \&

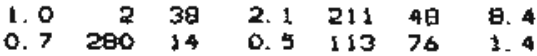

$$
\text { at }
$$

$66 \quad 0.44$

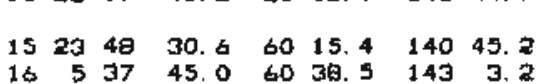
is 74 22. 2 69 21.4 is 3.4 自 43390 3. 5 M PMA 16 $\quad \begin{array}{lllllll}55 & 44.9 & 60 & 25 & 5 & 141 & 14.9\end{array}$ $\begin{array}{rrrrrrrr}16 & 10 & 30 & 55.0 & 60 & 15.3 & 140 & 44.4 \\ 16 & 11 & 0 & 4.9 & 60 & 28.3 & 143 & 10.9\end{array}$ 1611 o 97 60 20.1 143 9.4 16 1142 32 1 60 21, 141 22. 32.6 1. 3 10.3 a. 1,10 7.21 .51 6. 7 1. 1 10.0 1.7. $\begin{array}{llll}3 & 140 & 71 & 0.20\end{array}$ $\begin{array}{rrrr}124 & 77 & 0.24 \\ 149 & 220 & 0.46\end{array}$ $\$ 117 \quad 59 \quad 0.26$ $\begin{array}{llll}5 & 125 & 61 & 0.29 \\ 2 & 141 & 85 & 0.21\end{array}$ $\begin{array}{lllllll}16 & 1342 & 10.0 & \text { bo } & 16.8 & 141 & 13.7\end{array}$

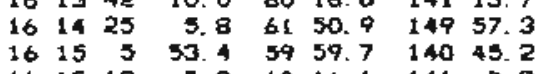
$\begin{array}{llllllll}16 & 15 & 5 & 33.4 & 59 & 59.7 & 140 & 45.2 \\ 16 & 15 & 9 & 60 & 16.1 & 141 & 0.8\end{array}$

15. 4 1. $7 \quad 12$ $7.90 .9 \quad 6 \quad 1 \quad 162 \quad 700.29$ 161794 22. $\begin{array}{lllllll}47.3 & 1.7 & 12 & 9 & 127 & 62 & 0.15 \\ 4 & 21 & 4 & 161 & 73 & 0.36\end{array}$ 1. 5 1.6A 911 $\begin{array}{lllllll}0.9 & 348 & 39 & 1.3 & 198 & 47 & 1.8\end{array}$

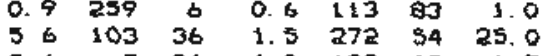

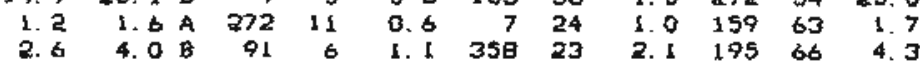
$161740 \quad 1.9 \quad 6025.214031 .5$ $5.2 \quad 2.0 \quad$ it 5 4 15 76 0.42 $\begin{array}{lllll}2.2 & 1.2 & A & 336 & 15\end{array}$ 2. $91.9 \mathrm{~B} 273$ $\begin{array}{llll}1.3 & 3.4 & 8 & 8.8 \\ 1.2 & 2.2 & 3 & 3\end{array}$

2. $2 \quad 77 \quad 36 \quad$ D. 9227 so 1.2 $\begin{array}{lllllll}2.5 & 273 & 24 & 5.2 & 129 & 63 & 25.0\end{array}$ $1.2176 \quad 7 \quad 0.7 \quad 339$ a2 3.4 $\begin{array}{lllll}1.4 & 2.4 & A & 112 & 2\end{array}$

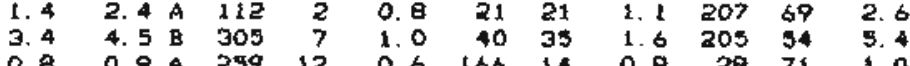

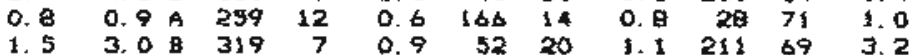

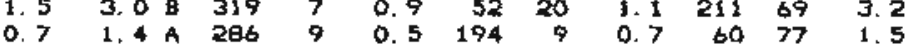

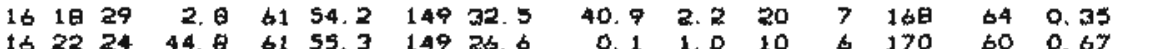

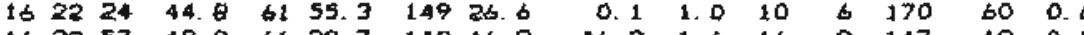

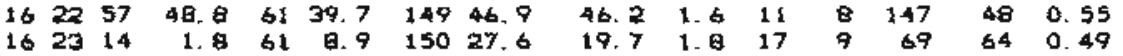

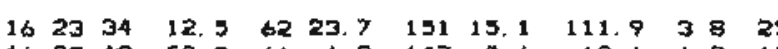

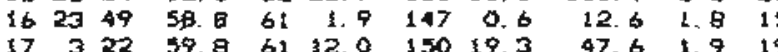

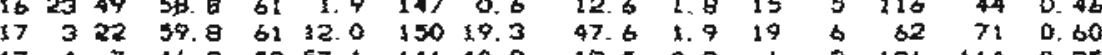

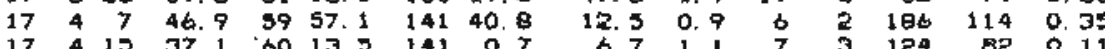

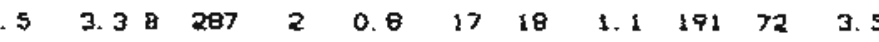

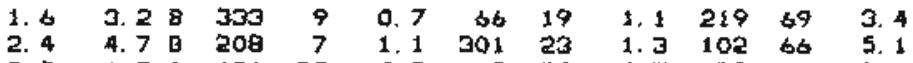

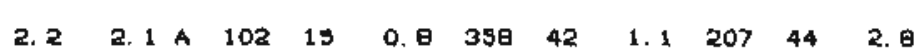

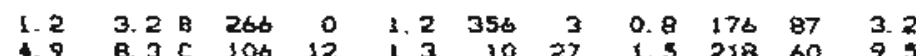

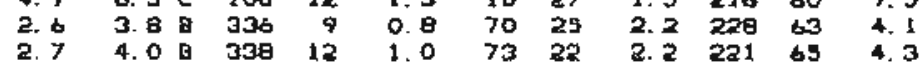

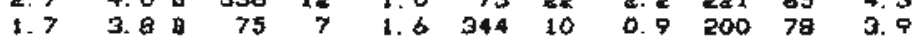

1. $3 \quad 2.2$ A 330 is $0 . \mathrm{O}$ b4 is $1.1199 \quad 68$ $\begin{array}{lllllllllll}5.2 & 2.5 & \text { A } & 107 & 6 & 0.9 & 14 & 24 & 1.9 & 210 & 69 \\ 10 & 2.5 & 5.3 & 113 & 16 & 0.9 & 327 & 71\end{array}$

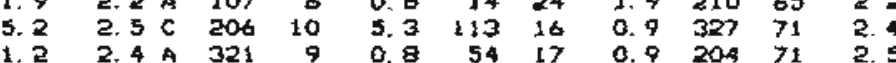
$\begin{array}{lllllllllll}1.2 & 2.4 A & 321 & 9 & 0.8 & 54 & 17 & 0.9 & 204 & 71 & 2.5 \\ 1.6 & 2.96 & 190 & 6 & 1.5 & 99 & 7 & 0.9 & 320 & 91 & 2.9\end{array}$ $1.62 .59286 \quad 5 \quad 0.8 \quad 17 \quad 16 \quad 1.4 \quad 179 \quad 73 \quad 3.0$

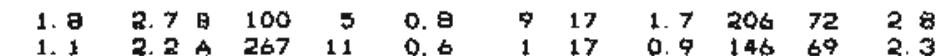

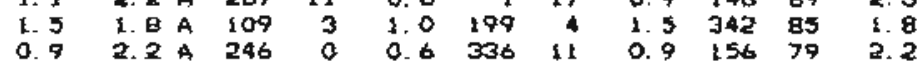

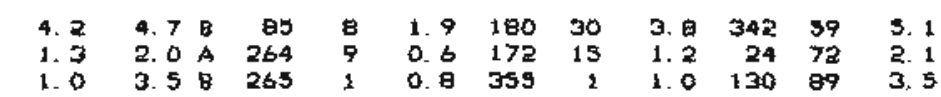

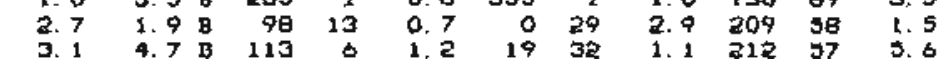


㞸

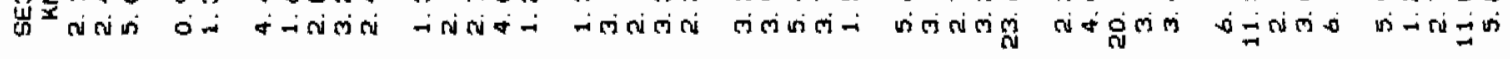

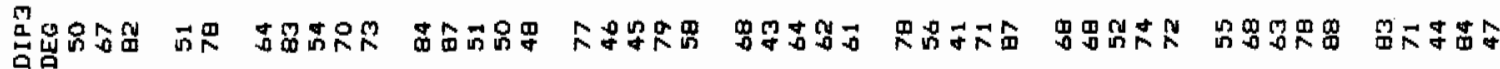

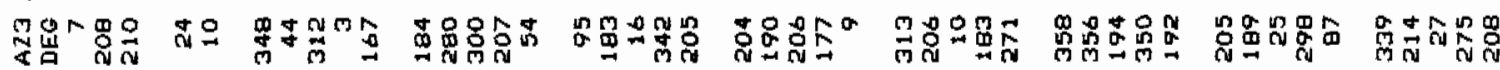
Cy

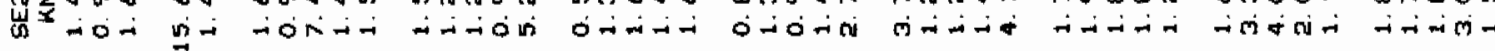

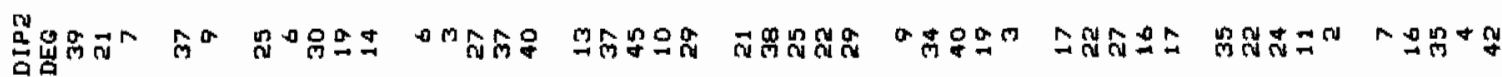

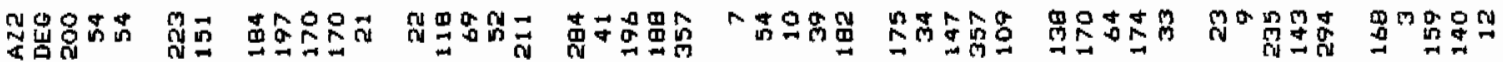

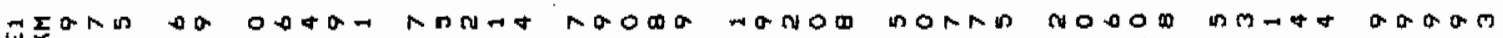
ज̆

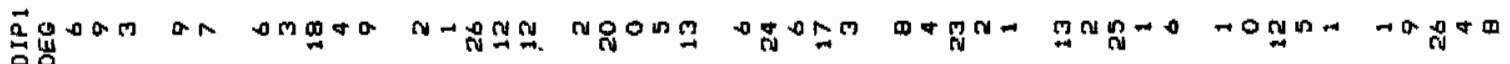

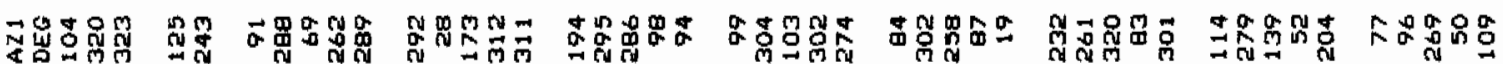

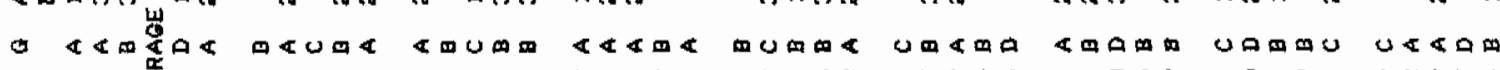

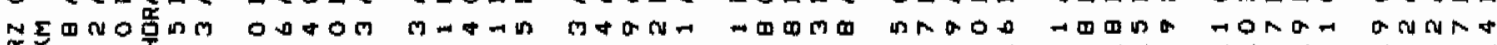
条

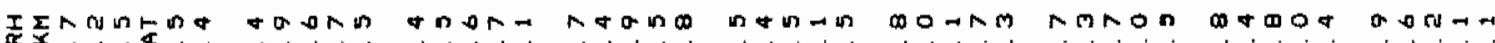

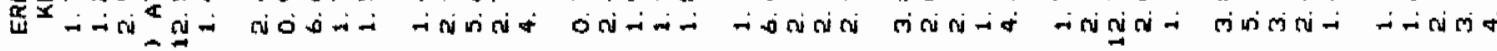

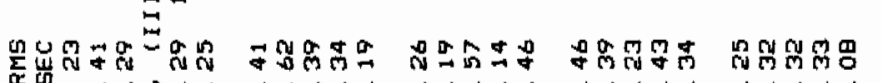
000-jo0 00000 00000 0000000000

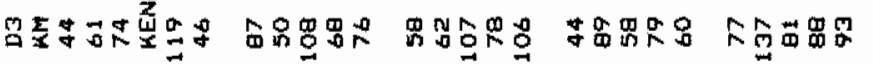

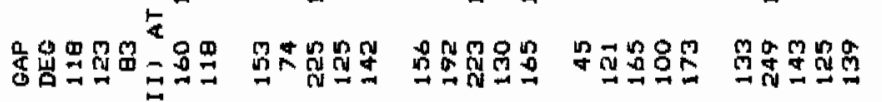

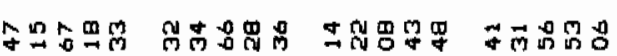

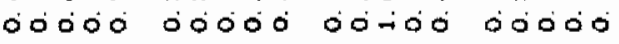

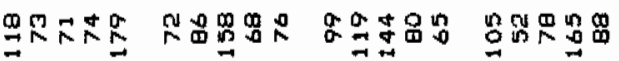
$0 \rightarrow \infty n$

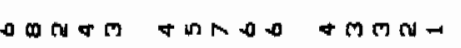

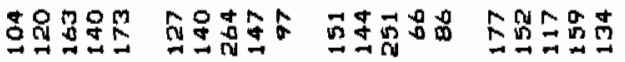
2

(1)
(1) : 2

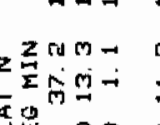

范品

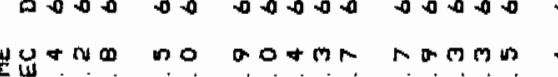
窵

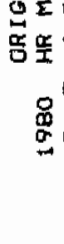

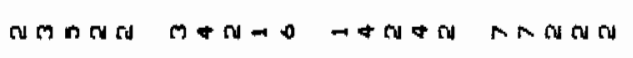

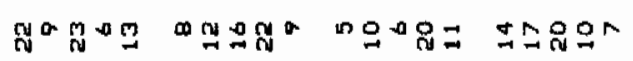
nM-As $\quad$ +

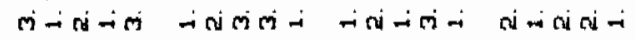

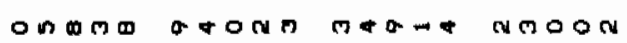

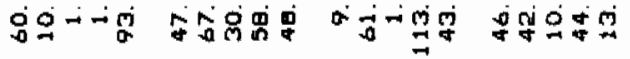

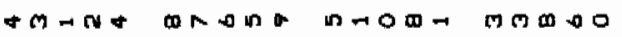

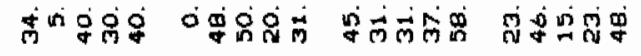

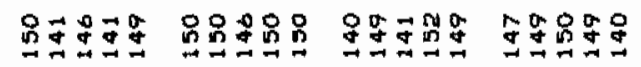
onm-a doma tomora momon

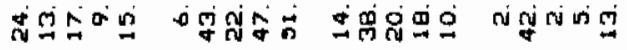

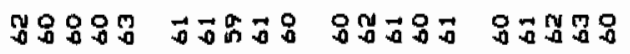

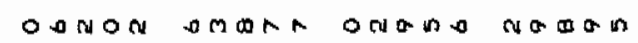

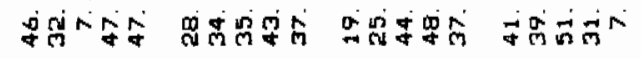

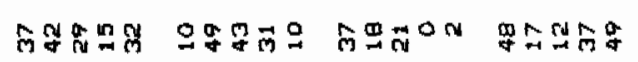

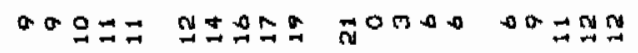

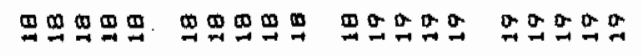


DRIGIN TIME LAT N LDNG W DEPTH MAG NP NG GAF DJ RM 1980 HR MN SEC DEG MIN DEG MIN KM 3.4 to DEG MM SEC $\begin{array}{lllllll}19 & 1355 & 20.4 & \text { to } 12.2 & 141 & 21.3\end{array}$ 171450 30. 3 61 $22.7 \quad 14637$.

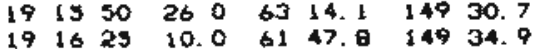
19 16 $29 \quad 20.2$ b2 $15.4 \quad 148 \quad 10.6$

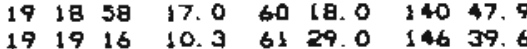
191916 10.3 $6129.0 \quad 146 \quad 39.6$ $3.5 \mathrm{MB}$

1922539.56011 .914058 .4

$20 \quad 146 \quad 30.7 \quad 62 \quad 0.6 \quad 147 \quad 40.7$ $20251 \quad 52.3$ 62 47.2 1.49 22. 20050 6. 56014.314050 .1 20539399 60 $12.2 \quad 1410.2$

$\begin{array}{llllllll}20 & 7 & 9 & 44.3 & 61 & 32.1 & 149 & 22.3\end{array}$ $\begin{array}{rrrrrrrr}20 & 11 & 53 & 53.1 & 60 & 16.4 & 141 & 2 . \\ 20 & 12 & 5 & 48.7 & 60 & 16.6 & 141 & 2 .\end{array}$

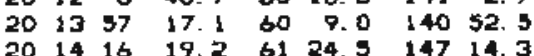
$202144 \quad 14.2$ 61 $49.2 \quad 14936$. $2022 \quad 34 \quad 16.7$ b1 18.5 150 37.2

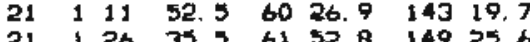

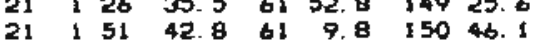

212523.7 68 B. $1 \quad 150+5.6$ $21 \quad 249 \quad 5.0 \quad 5958.9 \quad 14146.5$ 21900392 60 59.1147 B. $21926 \quad 25.9$ 60 27.514449 .0

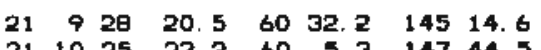
$\begin{array}{rrrrrrrr}21 & 10 & 25 & 23.2 & 60 & 5.3 & 147 & 44.5 \\ 21 & 10 & 54 & 41.4 & 60 & 26.5 & 145 & 0.6\end{array}$ 2110 96 42.0 61 30. 1 146 23.6

$\begin{array}{lllllll}6.7 & 1.1 & 5 & 2 & 151 & \text { 日2 } & 0.20\end{array}$

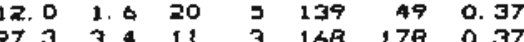
$\begin{array}{lllllll}97.3 & 3.4 & 11 & 3 & 168 & 176 & 0.37 \\ 40.9 & 3.2 & 26 & 2 & 158 & 61 & 0.32\end{array}$

\section{$39.0257 \quad 23 \quad 4 \quad 104$ 69 0.41}

$\begin{array}{lllllll}\text { 1.3. } & 1.24 & 2 & 2 & 192 & 80 & 0.03 \\ 27.9 & 3.3 & 31 & 2 & 67 & 48 & 0.62\end{array}$

$\begin{array}{lllllll}11.3 & 2.1 & 13 & 4 & 223 & 62 & 0.14\end{array}$

$\begin{array}{lllllll}30.2 & 2.0 & 17 & \text { b } & 173 & 78 & 0.73\end{array}$

39. $\begin{array}{llllllll}2.5 & 12 & 3 & 243 & 134 & 0.42\end{array}$

39. $3 \quad 1.7$ 10 6 i66 $43 \quad 0.20$

$\begin{array}{rrrrrrr}11.4 & 1.3 & 9 & 7 & 1.37 & 71 & 0.18 \\ 7.3 & 1.6 & 9 & 3 & 1.2 & 01 & 0.25\end{array}$

$\begin{array}{rrrrrrr}4.0 & 1.6 & 12 & 4 & 169 & 35 & 0.66 \\ 3.3 & 0.9 & 5 & 2 & 166 & 100 & 0.13\end{array}$

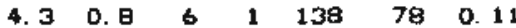

$\begin{array}{lllllll}22.4 & 1.24 & 3 & 1 & 273 & 127 & 0 . \\ 19.0 & 1.8 & 20 & 4 & 06 & 59 & 0.41\end{array}$

$\begin{array}{lllllll}44 . & 2.1 & 16 & 6 & 160 & 67 & 0.37 \\ \text { 62. } 3 & 2.1 & 23 & b & 101 & \text { a5 } & 0.36\end{array}$ 1.6 $1.63 \quad 3 \quad 208$ 150 0.02 37.01 .619 13 166 56 0.53

18. 9 2.0 0

13. 2.0 .4252

\begin{tabular}{lll}
74 & 0.05 \\
\hline & 75 & 0.43
\end{tabular}

13. $7 \begin{array}{llllll}1,8 & 24 & 14 & 98 & 41 & 0.43\end{array}$

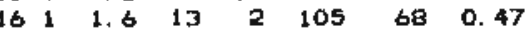

10. 2 0.

$\begin{array}{lllll}31 & 4 & 173 & 77 & 0.32 \\ 9 & 95 & 0.53\end{array}$

23.2 lllllll

$\begin{array}{rrrrrrr}20.0 & 2.2 & 20 & 9 & 79 & 50 & 0.66 \\ 0.0 & 0.7 & 9 & 3 & 231 & 104 & 0.39\end{array}$

$\begin{array}{llllllll}21 & 11 & 39 & 43.7 & 61 & 30.1 & 146 & 25.3\end{array}$

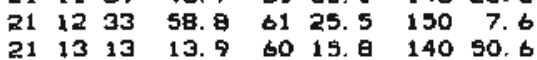

$21.9 \quad 1.720 \quad 8 \quad 78$ 50 0.55

$\begin{array}{rrrrrrr}16.4 & 1.1 & 8 & 5 & 125 & 86 & 0.50 \\ 10.3 & 1.9 & 20 & 7 & 62 & 40 & 0.33\end{array}$

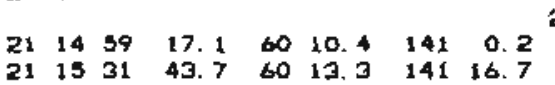

5 in ENRC

3. 1.2 क 2 134 $90 \quad 0.38$

$211546 \quad 30.4$ 60 $17.6 \quad 14044.3$

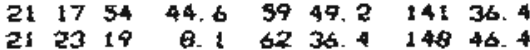

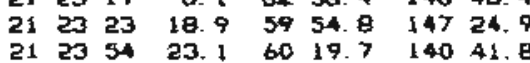

$\begin{array}{ccccccc}13.0 & 1.34 & 3 & 2 & 100 & 121 & 0.01 \\ 9.9 & 1.54 & 3 & 2 & 270 & 141 & 0.09\end{array}$

79.0 2. 5 23 20 10 127 \$15 0.60
ERH ERI O A21 DIP1 EEI AL2 DIP2 SE2 AZ3 DIP3 BE3 5. 3 KM DEO DEG KM DEG DEG KM DEG DEG KM

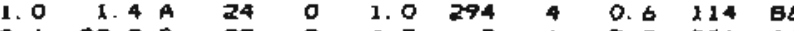

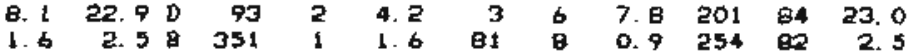

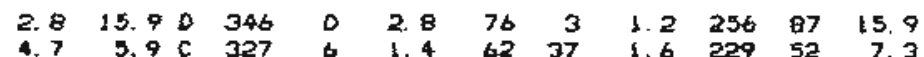

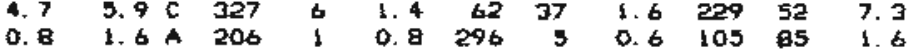

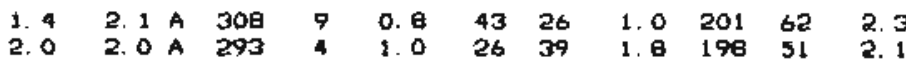

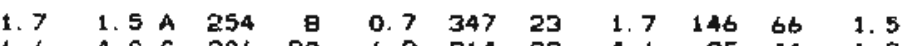

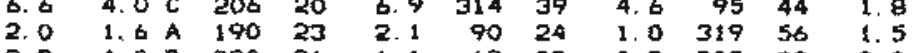

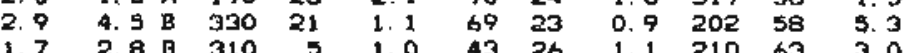

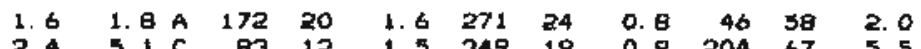

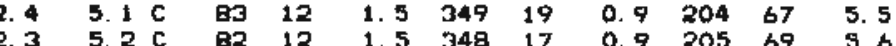

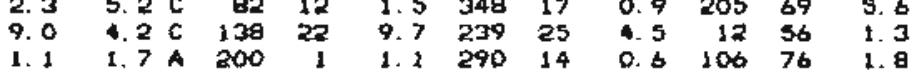

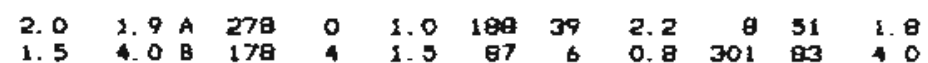

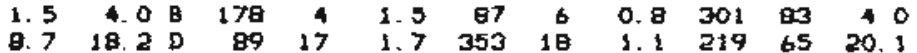

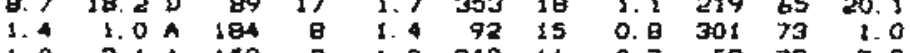
$1.02 .1 \mathrm{~A}$ lse 2 1.0 248 11 0.7 se 79 Z.2

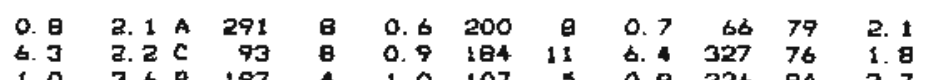

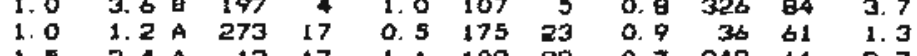

2. 4 1.7 A 182 27 25 76 $20 \quad 0930949$

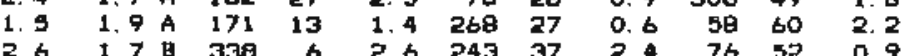

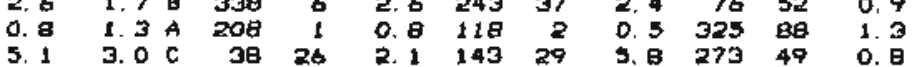

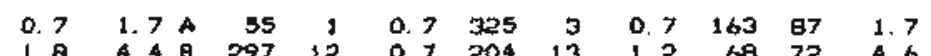
$\begin{array}{rrrrrrrrrrr}1.8 & 4.49 & 297 & 12 & 0.7 & 204 & 13 & 1.2 & 59 & 72 & 4.6 \\ 1.1 & 2.5 \mathrm{~A} & 306 & 7 & 0.8 & 39 & 16 & 0.9 & 193 & 72 & 2.6\end{array}$

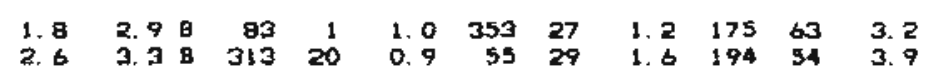

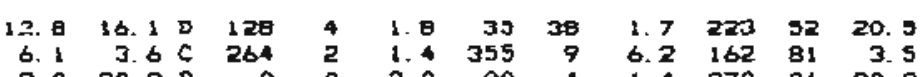

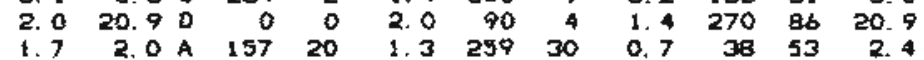
$\begin{array}{rrrrrrrrrrr}1.7 & 2.0 & 157 & 20 & 1.3 & 259 & 30 & 0.7 & 38 & 53 & 2.4 \\ 4 . t & 7.4 & 01 & 21 & 1.5 & 342 & 22 & 1.3 & 210 & 59 & 8.6\end{array}$ 
GOUTHERN ALASKA EARTHOUAKES, JAN = MAR 1990

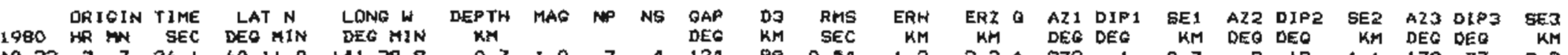
19DO SEC DEO MIN DEG HIN KM

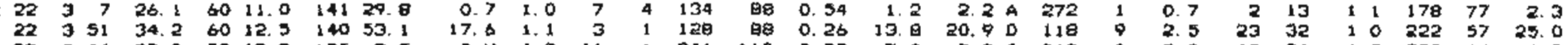

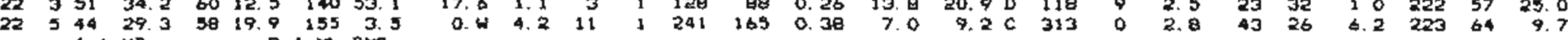

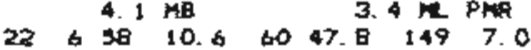

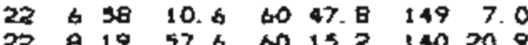

35. 7 2. 310

$7655 \quad 0.4$

$1.2 \quad 1.1 \% 909$

$\begin{array}{lllllll}0.7 & 205 & 34 & 1.2 & 339 & 42 & 1.1\end{array}$

$229541 . \$ 61151.3 \quad 14724.6$ $221729 \quad 31.460457$. 14740.1

$22 \quad 224150.7$ 60 0.1 145 37.1

13. 2. 2. 16 \& 190

$\begin{array}{llll}6.6 & 0.9 & 3\end{array}$

$\begin{array}{lllllll}7 & 32 & 36.4 & 60 & 3.4 & 140 & 125\end{array}$

23 日 9 2.6 60 3. 4 140 14.7

$231310 \quad 30.4 \quad 6013.6 \quad 14056.2$

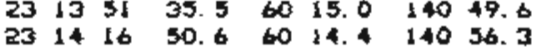

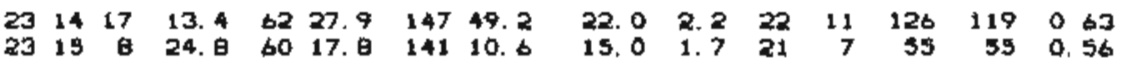
2315929.6 61 53.5 146 56. 2.4 ML EMRC

$\begin{array}{llllll}1.3 & 11 & 5 & 128 & 73 & 0.15\end{array}$

$\begin{array}{lllll}1.7 & 1.4 & A & 297 & 17\end{array}$

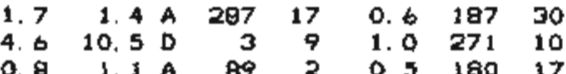

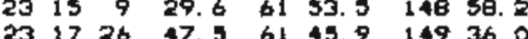
231726047.5 61 $45.9 \quad 14936.0$

$\begin{array}{llllllll}23 & 19 & 49 & 31.8 & 60 & 39.8 & 143 & 14.0\end{array}$

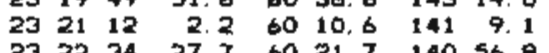

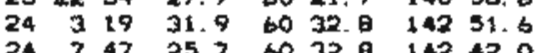

$\begin{array}{ll}35 & 0.71 \\ 38 & 0.46\end{array}$ $\begin{array}{rrrr}1.6 & 3.2 & A & 30 \\ 1.1 & 1.8 & A & 200\end{array}$

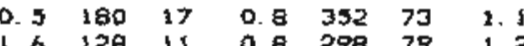
19. 2 1. $5 \quad 9 \quad 2 \quad \begin{array}{lllll}541 & 51 & 0.41\end{array}$ $\begin{array}{llll}\text { 2. } & 1.6 & \text { A } & 301 \\ \text { 3. } & 2.1 & \text { B } & 202\end{array}$ 3. 3 6. 1 C 120 $\begin{array}{rrrrr}1.1 & 1.9 & \text { A } & 322 & 8 \\ 2.7 & 4.7 & 8 & 97 & 9\end{array}$ $\begin{array}{rrrrrr}0.6 & 210 & 20 & 2.1 & 37 & 62 \\ 1.0 & 12 & 13 & 3.3 & 183 & 77\end{array}$ $\begin{array}{lll}9.0 & 298 & 79 \\ \text { 1. } 0 & 200 & 74\end{array}$ 2.
1. 2
1. 9 $241317 \quad 52$. 日 $60 \quad 27.3 \quad 14132.3$

$\begin{array}{lllllll}19.6 & 1.1 & 7 & 2 & 87 & 73 & 0.50\end{array}$ $\begin{array}{lllllll}13.9 & 0.6 & 7 & 1 & 141 & 08 & 0.45\end{array}$ $\begin{array}{rrrrrrr}10.1 & 1.2 & 7 & 4 & 139 & 71 & 0.94 \\ 162 & 1.5 & 14 & 5 & 83 & 61 & 0.74\end{array}$ 1.6 1.4 A 6927 $\begin{array}{llll}1 & 2.0\end{array}$ $\begin{array}{lrlllll}0.7 & 54 & 17 & 0.9 & 200 & 71 & 2.0 \\ 1.4 & 3 & 27 & 1.0 & 204 & 61 & 5.3\end{array}$

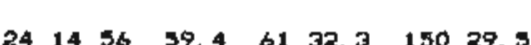
$11.9 \quad 1.920 \quad 6 \quad 57$ 5 ML EMRC $\begin{array}{llllllll}24 & 14 & 56 & 59.4 & 61 & 32.3 & 150 & 29.5 \\ 24 & 16 & 56 & 49.4 & 60 & 3.1 & 140 & 44.3\end{array}$

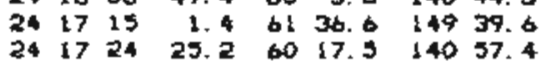
$2418 \quad 7 \quad 54.4$ 60 55.014940 .0

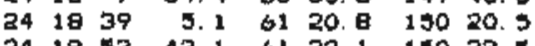
$\begin{array}{llllllll}24 & 18 & 53 & 43.1 & 61 & 22.1 & 150 & 23.5 \\ 24 & 21 & 97 & 33.7 & 60 & 12.4 & 140 & 59.7\end{array}$ 24231120.2 60 22.4 141 41.

60.91 .7

$\begin{array}{llllll}3 & 5 & 3 & 147 & 97 & 0.37\end{array}$ $\begin{array}{rrrrrrr}37.5 & 1.6 & 12 & 5 & 161 & 41 & 0.40 \\ 6.3 & 0.9 & 0 & 3 & 160 & 104 & 0.18\end{array}$ 41.91 .20

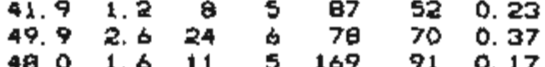
$\begin{array}{rrrrrrr}48.0 & 1.6 & 11 & 5 & 169 & 91 & 0.17 \\ 5.0 & 0.7 & 5 & 2 & 163 & 100 & 0.12\end{array}$ 24231120.2 60 22. 4141441.5

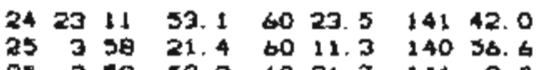

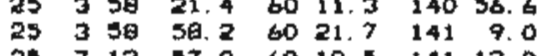
$\begin{array}{llllllll}25 & 7 & 13 & 57.0 & 60 & 16.5 & 141 & 12.0 \\ 25 & 7 & 47 & 45.7 & 64 & 19.3 & 145 & 13.3\end{array}$ 747 45.7 6419.3 . 145 13.3

$20.6 \quad 1.0 \quad 6 \quad 3 \quad 139$ b6 0.35 $\begin{array}{lllllll}3.7 & 0.7 & 5 & 4 & 149 & 104 & 0.12 \\ 5.1 & 0.9 & 3 & 3 & 169 & 125 & 0.12\end{array}$ 1. $1.1 \quad 6 \quad 3$ 152 1960.22

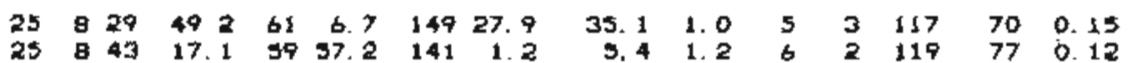
$\begin{array}{rrrrrrrr}25 & 8 & 43 & 17.1 & 59 & 37.2 & 141 & 1.2 \\ 25 & 9 & 7 & 17.5 & 61 & 29.0 & 140 & 27.9\end{array}$

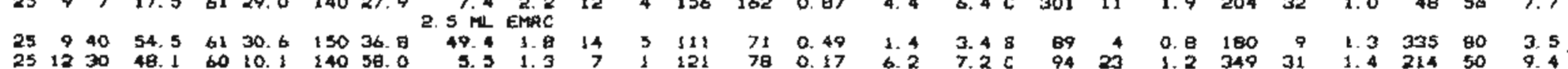
$2.04 .80 \quad 30010-1.7$

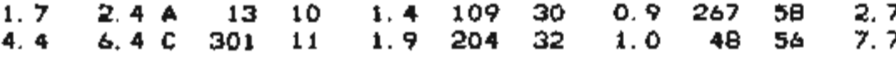

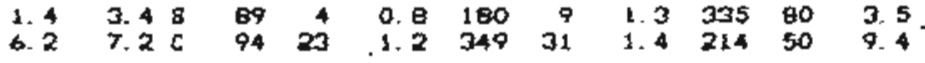


Mgookon moo an oo nom trmam

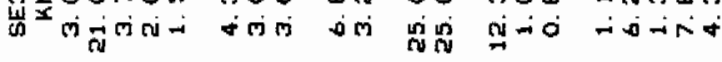

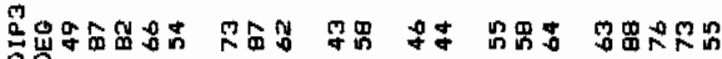

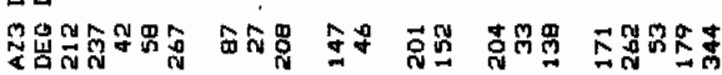
Cy पूँ

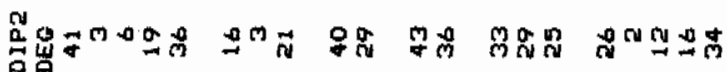

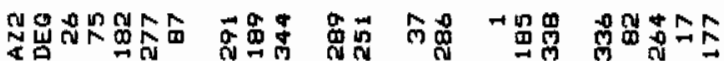

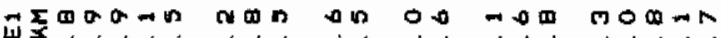

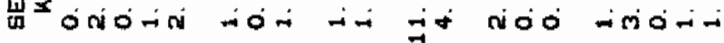

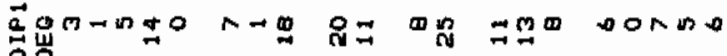

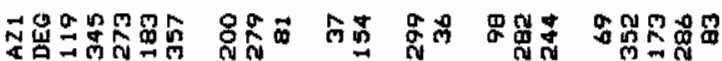

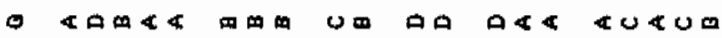

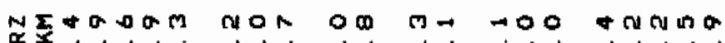

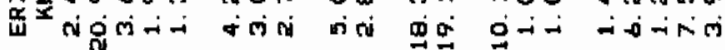

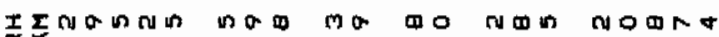
एँ

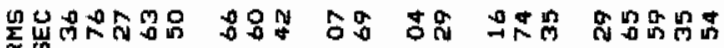

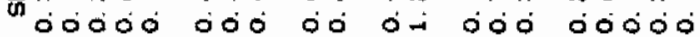

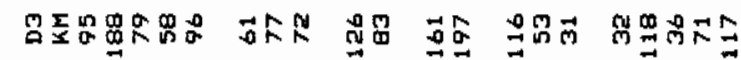

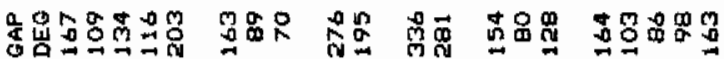
势 g a inot ing to an mot

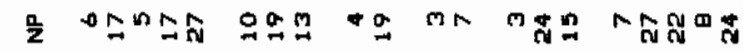

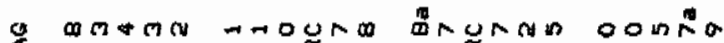
एक I oanmo on

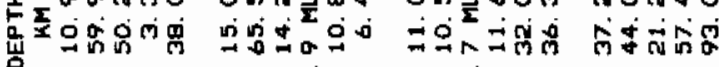

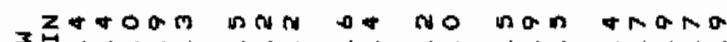

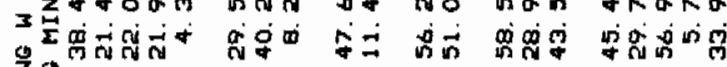

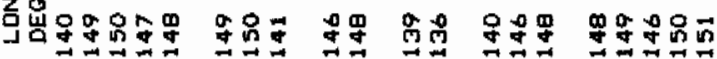

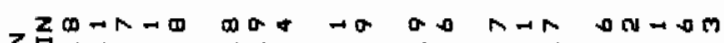

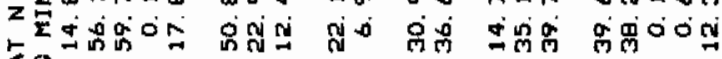

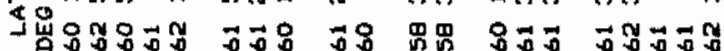

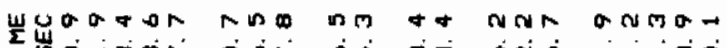

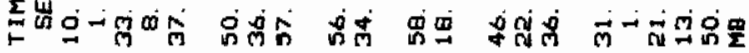

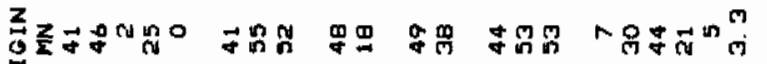

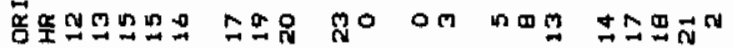

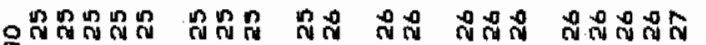
$\stackrel{0}{\frac{0}{2}}$

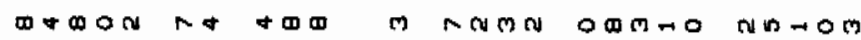

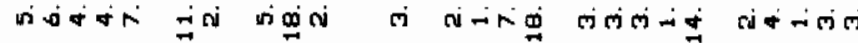

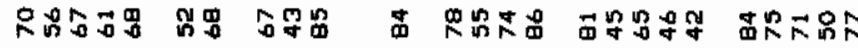

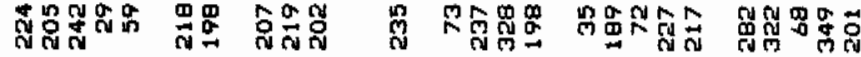

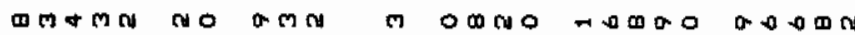

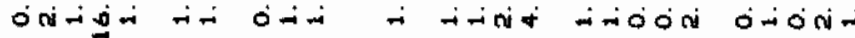

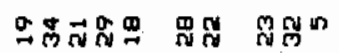

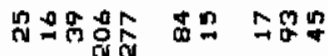

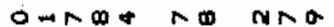
-iनion -io -io

$0+\infty-g g^{\circ}+g^{\infty}$

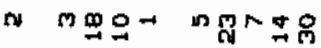

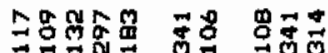

음

u umac u\& mom

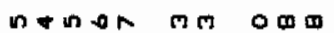

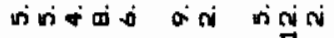

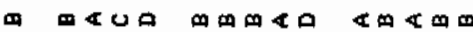

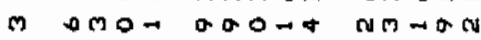

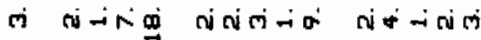

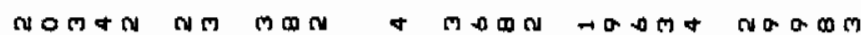

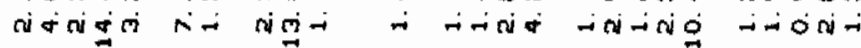

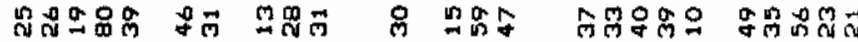

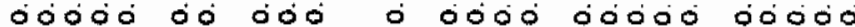

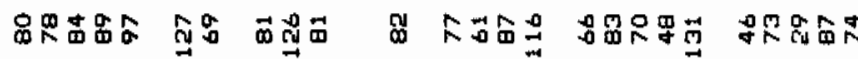

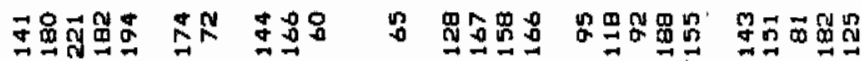

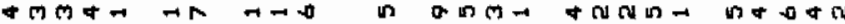

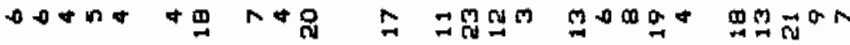

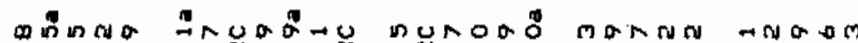

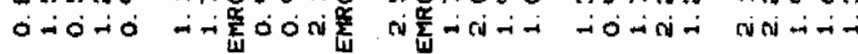

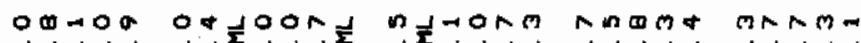
ம்

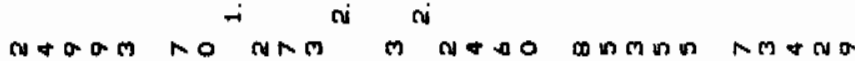

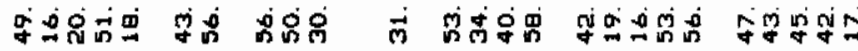

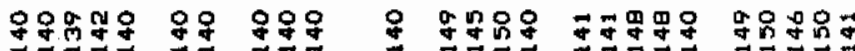

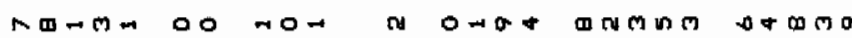

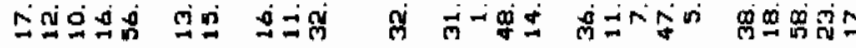

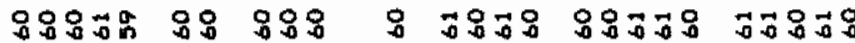

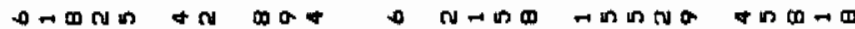

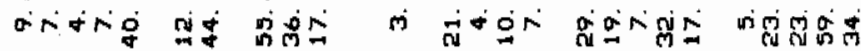

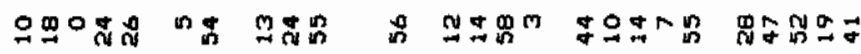
๙

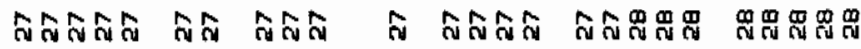




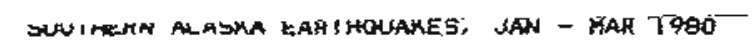

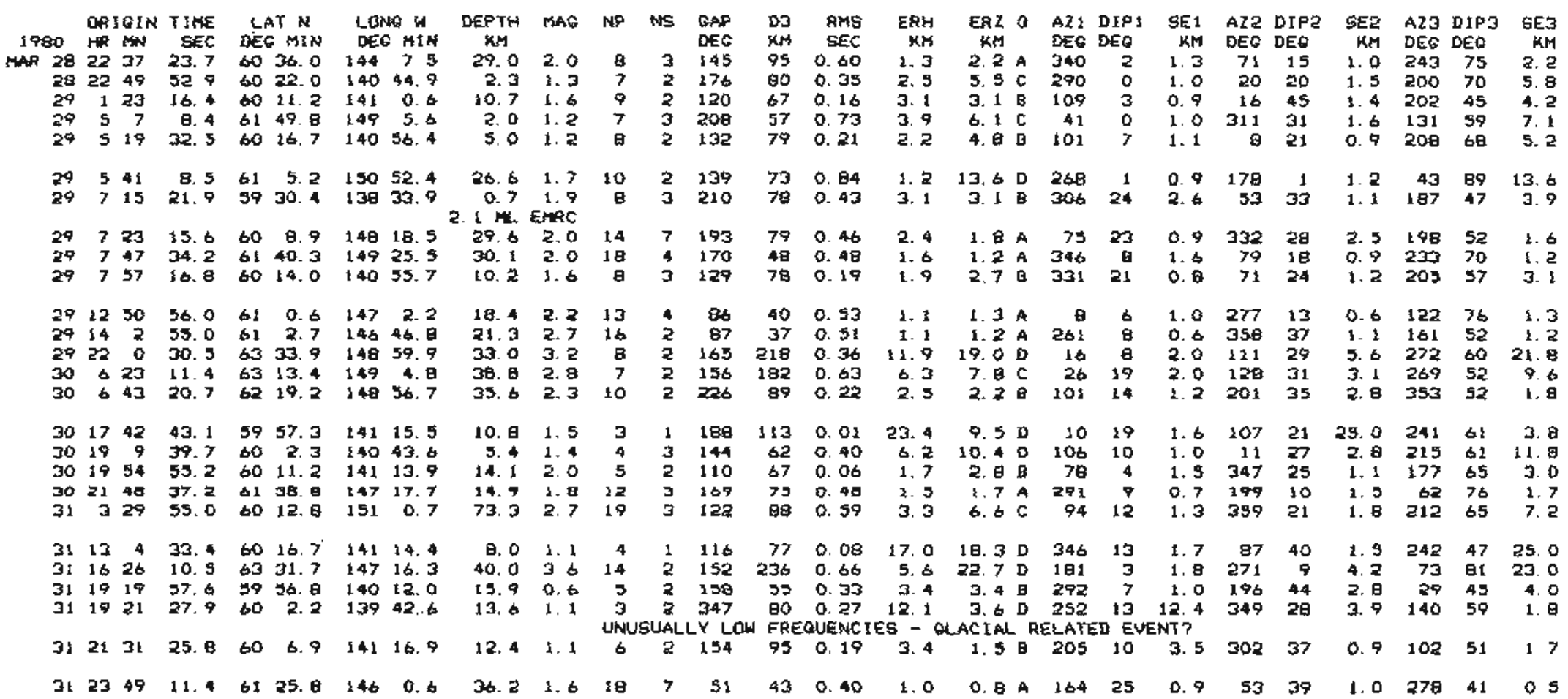


$\left[\begin{array}{ll}- & -1\end{array}\right.$ 